

\section{DISCLAIMER}

This document was prepared as an account of work sponsored by an agency of the United States Government. Neither the United States Government nor the University of California nor any of their employees, makes any warranty, express or implied, or assumes any legal liability or responsibility for the accuracy, completeness, or usefulness of any information, apparatus, product, or process disclosed, or represents that its use would.not infringe privately owned rights. Reference herein to any specific commercial product, process, or service by trade name, trademark, manufacturer, or otherwise, does not necessarily constitute or imply its endorsement, recommendation, or favoring by the United States Government or the University of California. The views and opinions of authors expressed herein do not necessarily state or reflect those of the United States Government or the University of California, and shall not be used for advertising or product endorsement purposes.

This report has been reproduced

directly from the best available copy.

Available to DOE and DOE contractors from the

Office of Scientific and Technical Information

P.O. Box 62, Oak Ridge, TN 37831

Prices available from (615) 576-8401, FTS 626-8401

Available to the public from the

National Technical Information Service

U.S. Department of Commerce

5285 Port Royal Rd.,

Springfield, VA 22161 


\section{DISCLAIMER}

Portions of this document may be illegible in electronic image products. Images are produced from the best available original document. 


\title{
Assessment of Sediment Monitoring at LLNL
}

\author{
Gretchen Gallegos
}

March 17, 1994 


\section{Table of Contents}

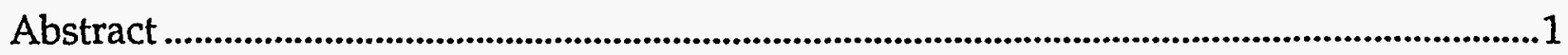

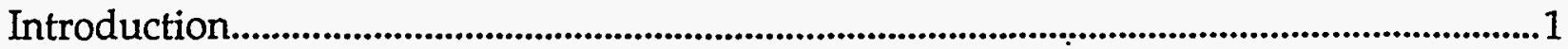

Method Review .....................................................................................................................

Annual Sampling............................................................................................................

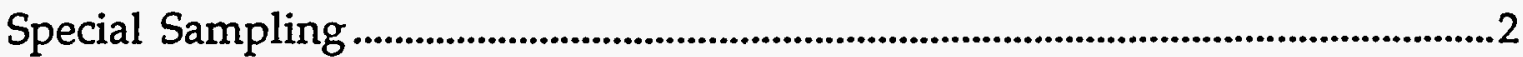

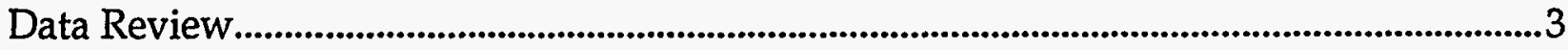

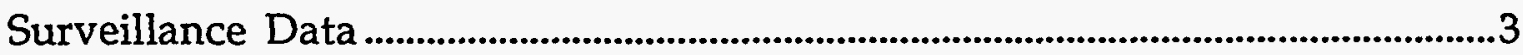

1989 Study Data ..................................................................................................................

1991 Study Data

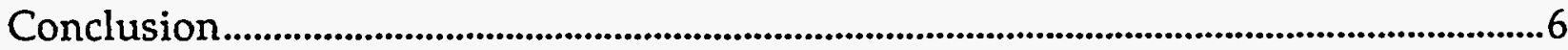

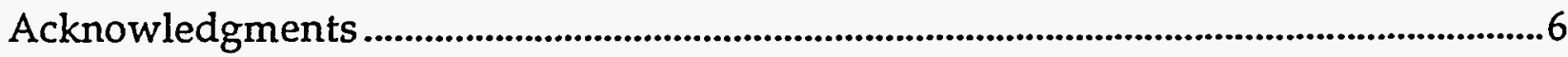

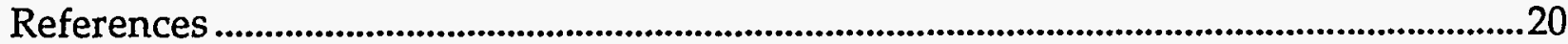

Appendix A: Letter Report for 1989 Sediment Study.......................................... Appendix B: 1991 Sediment Study Sampling Plan .......................................... B-1 to B-21 Appendix C: Surveillance Soils and Sediment Sampling Procedure.......... C-1 to C-16 Appendix D: Analytical Results from 1991 Sediment Study............................D-1 to D-9 


\section{List of Figures and Tables}

Figure 1: Surveillance Locations .................................................................................

Figure 2: $\quad$ Comparisons of ${ }^{232} \mathrm{Th}$ and Tritium data for 1991 study............................8

Figure 3: $\quad$ Comparisons of ${ }^{40} \mathrm{~K}$ and ${ }^{226} \mathrm{Ra}$ data for 1991 study...................................

Table 1: $\quad$ Metals values for sediment samples (mg/L), 1989 to 1992 .....................10

Table 2: $\quad$ Organic compounds and pesticides values for sediment samples,

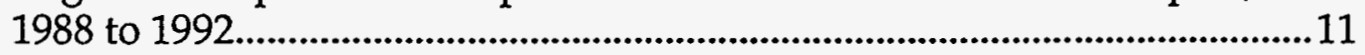

Table 3: $\quad$ Comparison of soils and sediment radionuclide data, 1988 to $1992 \ldots . .12$

Table 4: $\quad$ California waste extraction analysis metals from 1991 sediments study $(\mathrm{mg} / \mathrm{L})$...................................................................................................12

Table 5: $\quad$ ICAP metals analysis from 1991 sediments study on liquid samples (mg/L) ............................................................................................12

Table 6: $\quad$ ICAP metals analysis from 1991 sediments study on solid samples (mg/kg)

Table 7: $\quad$ Radionuclide analysis for 1991 sediment study $(\mathrm{pCi} / \mathrm{g}$, unless otherwise noted)

Table 8: $\quad$ Volatile organic compounds in 1991 study detected by EPA method 8270 and for which there are disposal limits, $\mathrm{mg} / \mathrm{kg}$ unless otherwise noted 15,16

Table 9: $\quad$ Pesticides detected in 1991 study in $\mathrm{mg} / \mathrm{kg}$ unless otherwise noted ...17

Table 10: $\quad$ Volatile organic compounds detected in 1991 study (EPA method $5030 / 8240$ ) for which there are disposal limits, $\mathrm{mg} / \mathrm{kg}$ unless otherwise noted

Table 11: $\quad$ Summary of materials found in the sediment studies above hazardous waste disposal limits. 


\title{
Assessment of Sediment Monitoring at LLNL Gretchen Gallegos
}

\begin{abstract}
Three separate sediment monitoring studies have been conducted at the
\end{abstract} Lawrence Livermore National Laboratory (LLNL) Livermore site. "Sediment" is defined here as finely divided solid materials that have settled out of an active stream or standing water. Sediment samples from all three studies were analyzed for a number of contaminants including ${ }^{239} \mathrm{Pu},{ }^{3} \mathrm{H}$, gamma emitting radionuclides, heavy metals, volatile organic compounds and pesticides. The analytical results for metals and organic compounds were compared to limits for disposal of hazardous waste, the tritium values were compared to drinking water standards, and the other radionuclides were compared to soils monitoring values. No tritium values were above (or were greater than $55 \%$ of drinking water standards), and no other radionuclides in sediments were above soils values. In all of the studies, only two metals, lead and mercury, and six organic compounds, benzo(a)-pyrene, Dieldrin, p, $\mathrm{p}^{\prime}$-DDT, Endosulfan I, endosulfan sulfate, and vinyl chloride were above waste disposal limits. Three of the high contaminants, mercury, benzo(a)-pyrene, and vinyl chloride, were found at one sampling location; the others were not connected by drainage channels or physical proximity to each other. Overall, a total of 247 samples were analyzed, and the sporadic identification of materials over disposal limits demonstrates that there is negligible contamination of sediment.

\section{Introduction}

Three separate sediment monitoring studies have been conducted at the Lawrence Livermore National Laboratory (LLNL) Livermore site. "Sediment" is used here to mean finely divided solid materials that have settled out of an active stream or standing water (it is not "sediment" in a geologic sense-by the geologic definition, the top 1000 meters at the site is sediment). In 1988, an annual sediment surveillance monitoring program was initiated to assist with environmental restoration efforts at locating areas of contamination (Holland, 1993). Sediment from catch basins, open drainage channels, and manholes were sampled in two other sampling efforts; one in 1989, in which 30 surface soil, drainage channel, and storm drain locations were sampled (see letter report of F. Hoffman to C. DeGrange, May 16, 1989, Appendix A) and one in 1991, in which 89 locations were sampled (see W. Schwartz report, August 1991, Appendix B). 
Sediment samples from all three studies were analyzed for a number of contaminants including ${ }^{239} \mathrm{Pu},{ }^{3} \mathrm{H}$, gamma emitting radionuclides, heavy metals, volatile organic compounds and pesticides. These data are reviewed here for significant trends that should be used in determining the need for continued studies of sediments at the LLNL Livermore site.

\section{Annual Sampling}

\section{Method Review}

Annual surveillance sampling of sediment is conducted according to a written procedure (see Appendix C). Permanent sampling locations were established in the Livermore site drainage channels and are shown on Fig. 1. Sampling locations were chosen to represent points of water flow on to and away from the site, and points where drainage channels met. Sediment is regularly deposited at location CDB, which is a siltation area for the Central Drainage Retention Basin. All other locations are generally scoured by runoff, and the deposition of material does not follow any particular pattern, making any conclusions about the source of deposited material difficult.

Quadruplicate samples were taken at each location in 1988, 1989 and 1990, and triplicate samples were taken in 1991 and 1992; a total of 138 samples were taken from 1988 to 1992. Samples were gathered in the late spring to summer months, between rainy seasons, and were taken $25 \mathrm{~cm}$ deep. The samples were submitted for analysis for halogenated volatile organic compounds (annual EPA Method 8010), aromatic volatile organic compounds, (EPA Method 8020), pesticides (EPA Method 8080), heavy metals, ${ }^{239} \mathrm{Pu}$, gamma emitting radionuclides, and tritium. .

\section{Special Sampling}

Two special sediment sampling studies have been undertaken in addition to the annual sediment monitoring. The first special study of sediment was in 1989. The locations for sampling were chosen to provide information for the LLNL Ground Water Project Baseline Public Health assessment. Twenty sediment locations were taken in open channel areas of the various drainages throughout the site and 10 surface samples were taken (see Fig. 1). The samples were taken $5 \mathrm{~cm}$ deep. They were analyzed for volatile organic compounds (EPA Method 8240), semi-volatile organic compounds, (EPA Method 8270), pesticides (EPA Method 8080), heavy metals, gamma emitting radionuclides, gross alpha radioactivity, gross beta radioactivity, and tritium.

The second special study of sediment took place in 1991. The locations of the second special sampling study were chosen to assess the entire LLNL Livermore storm drainage system. Starting at the highest elevation, sample locations were chosen at the down gradient junction of two intersecting drainage lines or at the junction of a drainage line and a network of drainage lines. Some locations were also chosen on the basis of past practices or known releases near a selected location. The depth of samples was not specified and could be taken to a depth of a "few feet"; the depths appears to have depended upon the depth of material present at any location. The samples were analyzed for volatile organic compounds (EPA Method 5030/8240), semi-volatile organic compounds, (EPA Method 8270), pesticides (EPA Method 8080), heavy metals, 
gamma emitting radionuclides, gross alpha radioactivity, gross beta radioactivity, and tritium.

\section{Surveillance Data}

\section{Data Review}

Surveillance sediment samples were arialyzed for three general types of contaminants, metals, organic compounds, and radionuclides. There are no specific standards regulating the amounts of contaminants in soils or sediments. To have some measure of whether the detected levels present any. cause for concern, it is useful to compare the levels with standards set for disposal of hazardous waste. Such limits include federal Resource Conservation and Recovery Act regulations, which are also codified in Title 22, California Code of Regulations sections 66261.33 and related section 66268.43. According to section 66261.33 (d): "any residue or contaminated soils, water or other debris resulting from the cleanup of a spill into or on any land or water of any commercial chemical product or manufacturing chemical intermediate having the generic name listed in subsection (e) or (f) of this section, ..." is a hazardous waste. Sections (e) and (f) list acute hazardous wastes and toxic wastes, and include RCRA category $\mathrm{P}$ and $U$ wastes; section 66268.43 lists the limits for these wastes.

With one exception, all metals analyses were below the limits for hazardous waste (see Table 1). The one analysis that was above the limits was for lead in 1989; $79 \mathrm{mg} / \mathrm{L}$ was found and the limit is $5 \mathrm{mg} / \mathrm{L}$. However, of the quadruplicate samples taken at this location (in accordance with the procedure), only one high value was found, the others were detection limits. Seven samples taken the following year and subsequent routine annual sampling also failed to show high values for lead at that location. This sampling location coincided with a location in for the 1989 special study, which also identified elevated values for chromium, lead, PCE, and Freon 11. This location was cleaned up (Thorpe et al., 1990).

Similarly, there have been no detections of organic chemicals or pesticides above waste limits (see Table 2). In fact, most chemicals were only detected sporadically and at levels barely above detection limits. Only one material was detected at above $40 \%$ of the standard for disposal of waste and only three were above $5 \%$ of the disposal standard.

There are no specific waste limits for radionuclides, so there is no convenient limit against which to compare the levels of radionuclides in sediments. However, the sediment samples are analyzed for almost the same radionuclides as the soil samples that are taken as part of the routine soils surveillance monitoring program. Both sets of samples are analyzed for ${ }^{239} \mathrm{Pu}$ and for gamma emitters (data are reported in Brekke et al. 1989; Gallegos et al., 1992, 1993; Sims et al. 1990, 1991. (Sediment samples are also analyzed for tritium.) A comparison of the results for routine soils and sediment samples shows that the data are not significantly different except for ${ }^{239} \mathrm{Pu}$ and ${ }^{137} \mathrm{Cs}$, which are higher for soils than for sediment (see Tables 3 and 4). This result is not unexpected because soils samples are taken $5 \mathrm{~cm}$ deep and sediment samples are taken $25 \mathrm{~cm}$ deep and ${ }^{239} \mathrm{Pu}$ and ${ }^{137} \mathrm{Cs}$ arise from surface deposition.

The surveillance soils samples were not analyzed for tritium, so sediment values cannot be compared to soils values. However, there is a California drinking water standard (22 California Code of Regulations Section 64443) for tritium of 20,000 pCi/L, 
and the values for tritium present in the moisture of the sediment can be compared to that standard (although a drinking water standard could be expected to be more stringent than a standard for materials present in soil). The highest value found for tritium was $4320 \mathrm{pCi} / \mathrm{L}$ at location ASE in 1989; this value is only $22 \%$ of the standard for drinking water.

\section{Study Data}

The data from the 1989 study were summarized in a letter report (all results from this study are reported in Thorpe et al. 1990, Appendix N). Of the 20 sediment samples taken, only two showed levels above limits for hazardous waste for metals. These samples were both taken in a storm drain culvert just east of Building 438 (the location was sampled in duplicate). The only metal detected above the limits for disposal of hazardous waste was lead. The values detected were $18 \mathrm{mg} / \mathrm{L}$ and $14 \mathrm{mg} / \mathrm{L}$ and the limit for disposal of hazardous waste is $5 \mathrm{mg} / \mathrm{L}$. As stated previously, this location was cleaned up (Thorpe et al., 1990). The location (near Building 438) was sampled as part of the surveillance monitoring program in 1990 to 1992, and no high values of lead were found again.

The 10 surface samples from the 1989 study also had one location with results above disposal limits. This sample was taken north of Building 515 and contained 1.4 $\mathrm{mg} / \mathrm{L}$ cadmium (limit $1.0 \mathrm{mg} / \mathrm{L}$ ), $29 \mathrm{mg} / \mathrm{L}$ copper (limit $25 \mathrm{mg} / \mathrm{L}$ ), and $8.5 \mathrm{mg} / \mathrm{L}$ lead (limit $5 \mathrm{mg} / \mathrm{L}$ ). It should be noted that this is in the area near waste disposal operations and is not in a drainage channel. Therefore, it should not be used to determine the need for a sediment surveillance monitoring program. The site where this sample was taken has also been cleaned up (Thorpe et al., 1990).

The 1989 study also showed four elevated values for tritium. Two values, 11,000 $\mathrm{pCi} / \mathrm{g}$ and $3000 \mathrm{pCi} / \mathrm{g}$ were at the same sample location where high values for lead were detected. This location also showed one high tritium values in surveillance monitoring, $4100 \mathrm{pCi} / \mathrm{g}$ in 1991 . Two other locations showed values of $2000 \mathrm{pCi} / \mathrm{L}$. Nonetheless, the highest value still is only $55 \%$ of the drinking water standard of $20,000 \mathrm{pCi} / \mathrm{L}$. In addition, this location has been cleaned up (Thorpe et al., 1990).

\section{Study Data}

The sampling locations of the 1991 study were chosen to provide an overall understanding of the deposition of contaminants in the drainage system, to that end 89 locations were sampled. Four types of samples were taken, background sediment samples, catch basin (and manhole) sediment samples, open channel sediment samples, and standing liquid samples. Of these sampling types, the open channel sediment samples are most similar to the sediment samples that are taken for surveillance monitoring.

The only metals detected above disposal limits in the 1991 study were lead and mercury, and none of these were detected in the open channel samples. Lead was detected in liquid samples at one location, CB-190T-49, at 3 times the disposal limit (see Tables 5 and D-2; and at one catch basin sampling location, CB-280D-24 (see Tables 6 and D-3), at 25 times the disposal limit. Mercury was only detected at one catch basin location, CB-320F-17, at 1.9 times the disposal limit (see Tables 6 and D-3). 
Six organic compounds and pesticides were found above disposal limits in the study; these compounds were benzo(a)pyrene, Dieldrin, p,p'-DDT, Endosulfan I, Endosulfan sulfate, and vinyl chloride (see Tables 8 to 10 and D-5 to D-7). Of these, one pesticide, Dieldrin, was in a background sample, BS-6-6, and was only slightly above the limit, i.e., $0.14 \mathrm{mg} / \mathrm{kg}$ was detected and the disposal limit is $0.13 \mathrm{mg} / \mathrm{kg}$. Benzo(a)pyrene, vinyl chloride, and p,p'-DDT were detected in catch basin samples. The first two were 6 times and 8.5 times the disposal limits of 8.2 and $33 \mathrm{mg} / \mathrm{kg}$, respectively, and were found at location CB-320F-17; the p, $\mathrm{p}^{\prime}$-DDT was found at CB$410 \mathrm{E}-44$ at 2 times the limit of $0.087 \mathrm{mg} / \mathrm{kg}$, and at location CB-190Q-10 $1 / 2$ at $0.098 \mathrm{mg} / \mathrm{kg}$, just above the limit. The Endosulfan compounds were both found in open channel samples, at location OCS-460-1-6 at 2.6 times the limit of $0.066 \mathrm{mg} / \mathrm{kg}$ and at location OCS-150-1 at 3.3 times the limit of $0.13 \mathrm{mg} / \mathrm{kg}$. Interestingly, location OCS-460$1-6$ is near surveillance location CDB and location OCS-150-1 is near surveillance location 4THA, and no endosulfan compounds have been detected at those locations in surveillance sampling before the 1991 study nor in the 1 year of surveillance sampling after. One reason for the difference in detections could be the difference in sampling depth. However overall, it can be seen that detections of any organic compound was sporadic, with the highest number of detections overall being for toluene with 16 detections, followed by fluoranthene and total xylene isomers with 15 detections. In addition, most detections were in the catch basin and liquid samples. The open channel samples were generally lower than the other sample types.

Because there are no standards available for easy comparison for radionuclides in hazardous waste, the radiological data from the 1991 study were compared to surveillance data for soils and sediments samples collected from 1988 to 1992. The data that were compared were ${ }^{40} \mathrm{~K}, 226 \mathrm{Ra},{ }^{232} \mathrm{Th}$, and tritium. Gross alpha and gross beta data were collected during the 1991 study, but not for surveillance samples, so there were no data for comparison. However, the results for gross alpha and gross beta were not very high; the highest gross alpha value was $18 \mathrm{pCi} / \mathrm{L}$ in a liquid sample and the highest gross beta value was $29 \mathrm{pCi} / \mathrm{g}$ in an open channel sample. The ${ }^{137} \mathrm{Cs}$ values were not compared because most of the ${ }^{137} \mathrm{Cs}$ values in the 1991 study were non-detects (see Table 7). The other nondetection values (i.e., those with more than $100 \%$ counting error) for the 1991 study were divided by 2 for the statistical comparison. In addition, three locations with particularly high detection limits for gamma-emitting radionuclides were excluded because they would dominate the resulting analysis; these locations were CB-410D-014, CB-510B-30, and CB-510D-20. An average value for each surveillance sediment location for each year was used so the surveillance locations would not be over represented.

The comparisons of the data show no differences among sampling location types for ${ }^{232}$ Th or tritium (see Figure 2), and show the catch basins results to be significantly less for ${ }^{40 \mathrm{~K}}$ and $226 \mathrm{Ra}$ (see Figure 3). The lower values in the catch basins may reflect the greater solubility of $40 \mathrm{~K}$ and ${ }^{226} \mathrm{Ra}$ compared to ${ }^{232} \mathrm{Th}$, i.e., these radionuclides may have flowed past the sediments in the water, rather than remain in the sediments. In any case, there appears to be no cause for concern that radionuclides are being selectively deposited in sediments. (In addition, all tritium values were well below the drinking water standard of 20,000 pCi/L; the highest open channel value was 6182 
$\mathrm{pCi} / \mathrm{L}$; the highest catch basin value was $2719 \mathrm{pCi} / \mathrm{L}$, and the highest liquid sample value was $6268 \mathrm{pCi} / \mathrm{L}$.)

\section{Conclusion}

LLNL has conducted three separate studies, including one a multi-year surveillance study and two single-year studies, and analyzed 247 samples of materials in surface drainages at the Livermore site. These studies were not based on the same approaches, and two were designed specifically to detect any interrelated contamination of the surface drainage at the site. The data for metals and organic compounds were compared to limits for disposal of hazardous waste, the tritium values were compared to drinking water standards, and the other radionuclides were compared to soils monitoring values. No tritium values were greater than $55 \%$ of drinking water standards, and no other radionuclides in sediments were greater soils values. In all of the studies, only two metals and six organic compounds were above waste disposal limits; these are presented in Table 11.

Although some sporadic contamination by lead and a few organic compounds was detected, overall it can be concluded that there is no systematic contamination of the surface drainage at the LLNL Livermore site, and, in fact, there is very little contamination at all. In view of these results and the fact that there is no regulatory requirement to monitor nonradiological materials in sediments, it is recommended that the nonradiological surveillance monitoring be discontinued. LLNL personnel will follow the development of the EPA Contaminated Sediment Management Strategy to determine the applicability of that strategy to LLNL sediments.

\section{Acknowledgements}

The author would like to acknowledge the work of William Schwartz in the development of the data analyzed in this paper. He was responsible for the extensive 1991 special study of sediment from planning, through sampling, to data acquisition. The completeness of the 1991 study has enabled this author to examine data that would otherwise have been unavailable. The author would also like to acknowledge the work of the sampling technicians who conducted the various sampling efforts including, but not limited to, Donald Ramsey, David Graves, Paris Althouse, Marion Heaton, and Rebecca Ward. 
- Surveillance locations 1991 Study

- 1989 Woter

O 1989 stormarain

1989 Soil

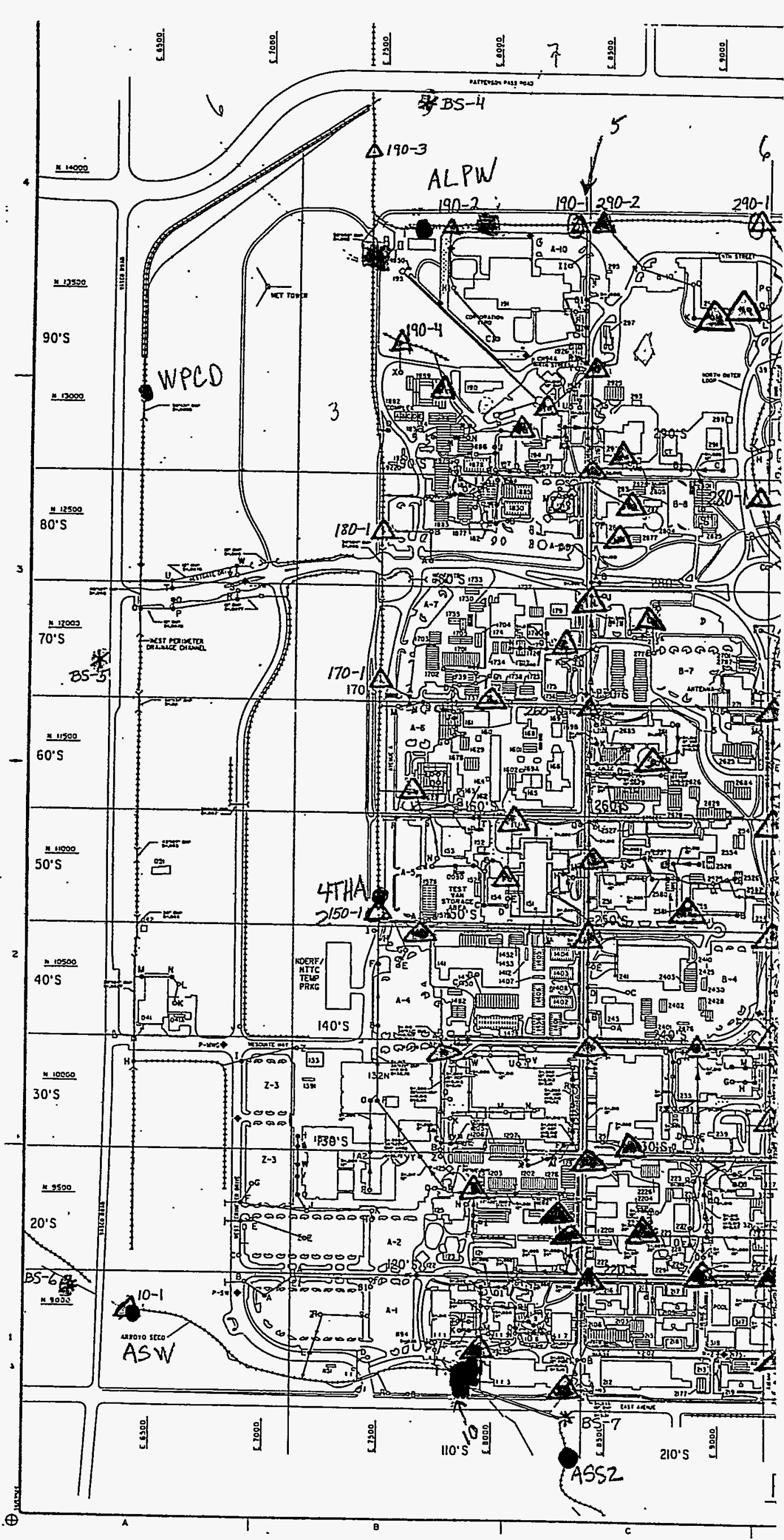




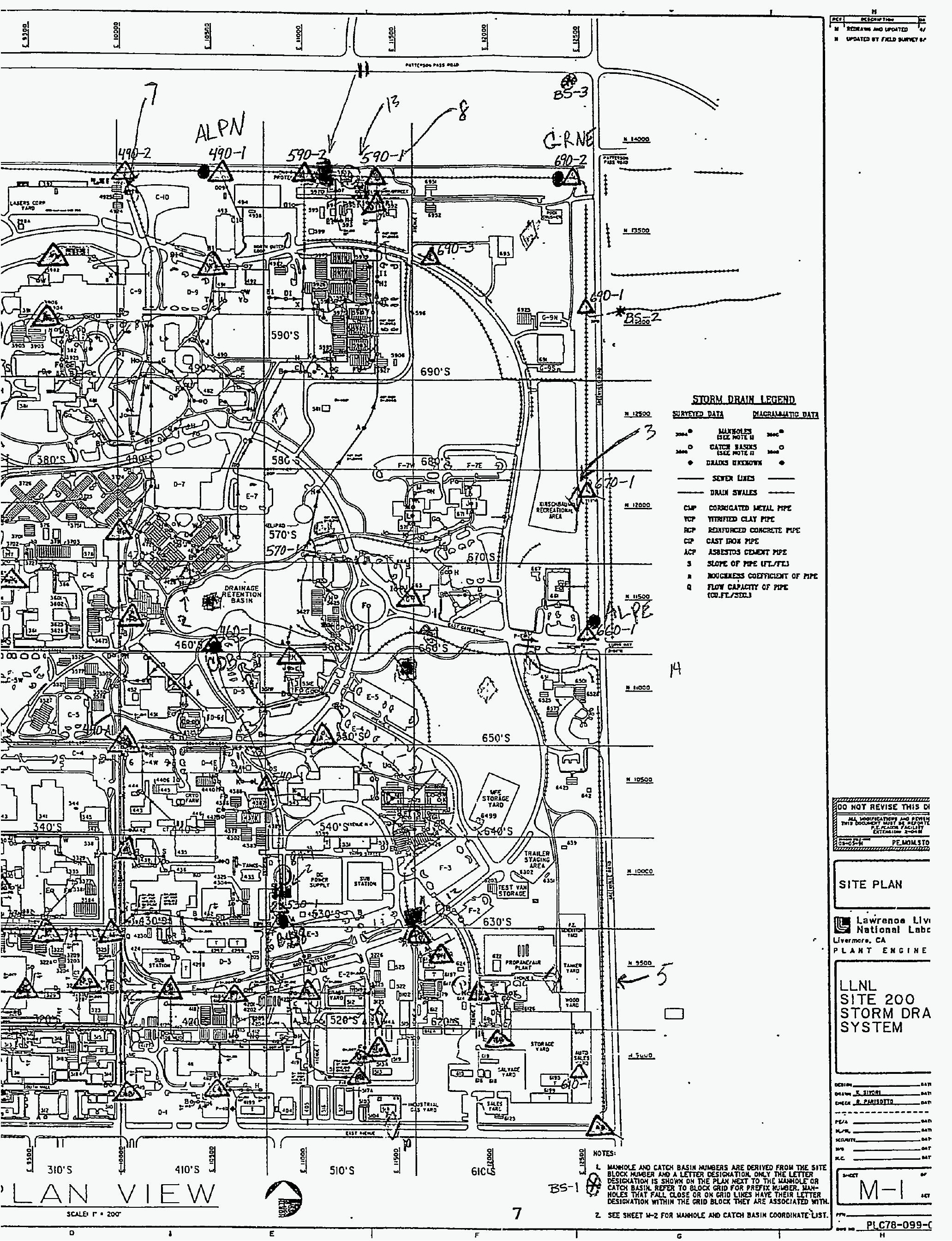



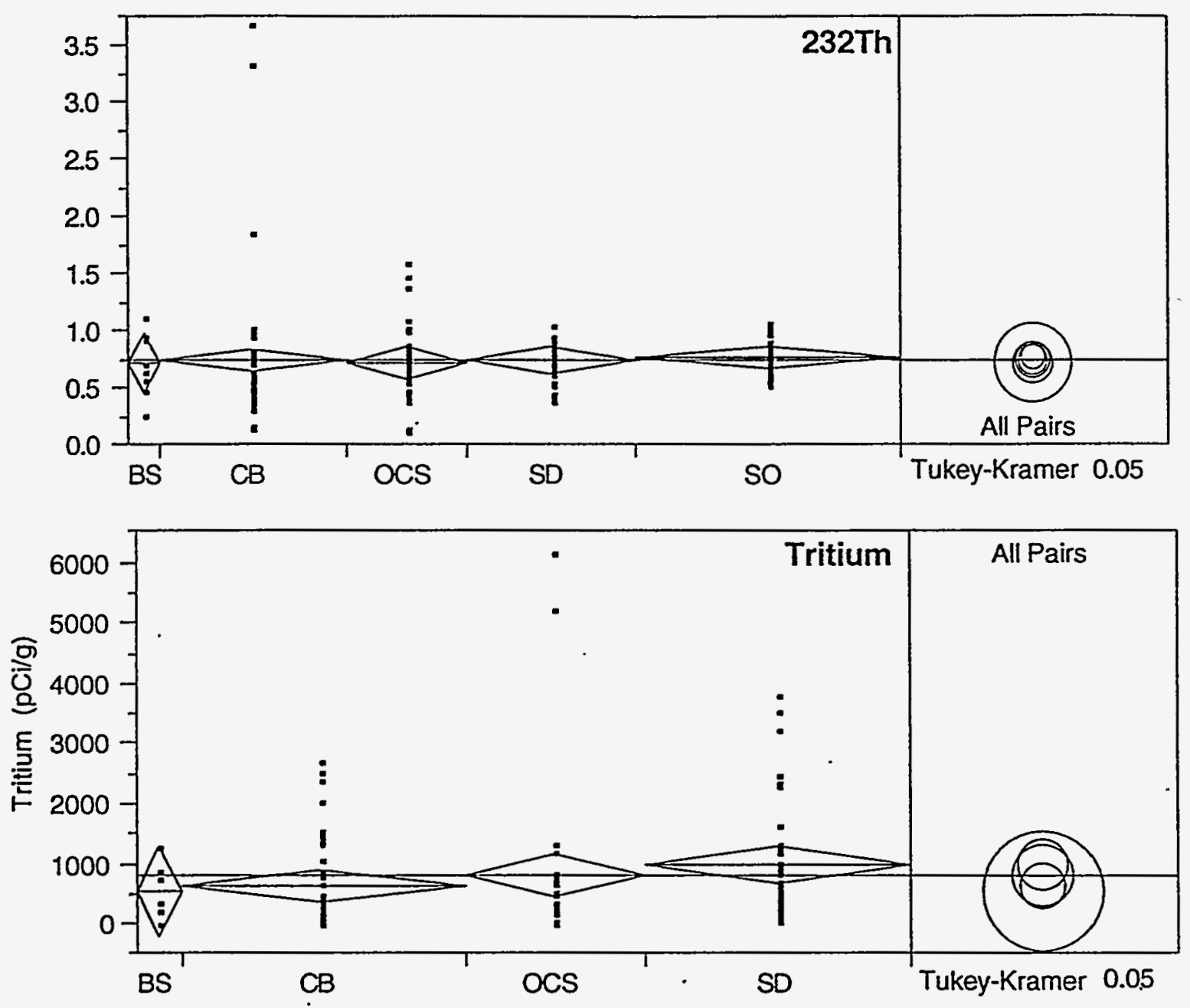

Figure 2. Comparisons of ${ }^{232} \mathrm{Th}$ and tritium data for 1991 study (BS = background samples, $\mathrm{CB}=$ catch basin samples, and $\mathrm{OCS}=$ open channel samples), surveillance sediment samples (SD), and surveillance soil samples (SO). The diamonds show the $95 \%$ confidence interval for the means; the line bisecting the diamond shows the mean for the group of data. The circles show significant differences; those that do not intersect, or for which the angle intersection is less than $90^{\circ}$, are significantly different. 

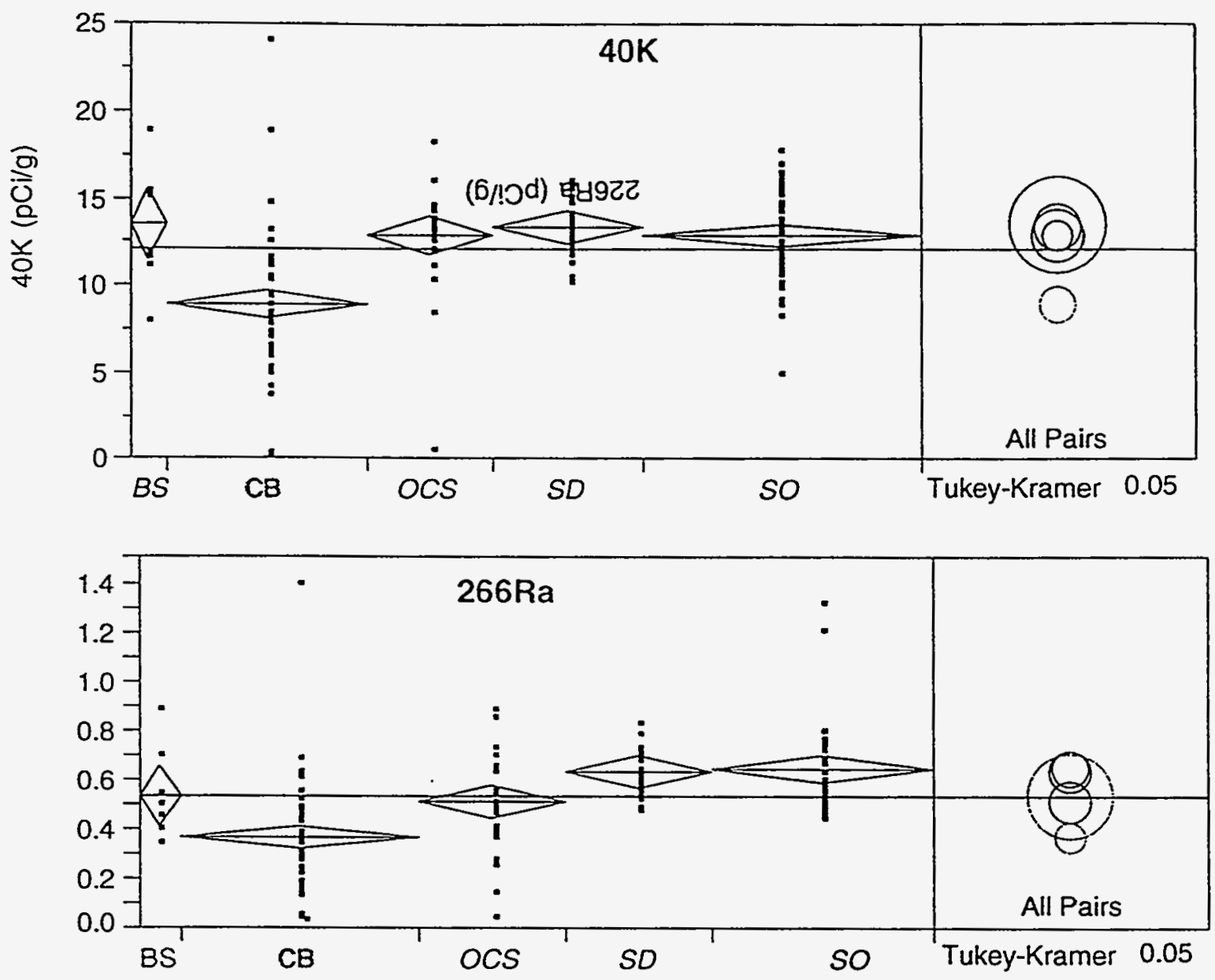

Figure 3. Comparisons of ${ }^{40} \mathrm{~K}$ and $226 \mathrm{Ra}$ data for 1991 study (BS = background samples, $C B=$ catch basin samples, and $O C S=$ open channel samples), surveillance sediment samples (SD), and surveillance soil samples (SO). The diamonds show the $95 \%$ confidence interval for the means; the line bisecting the diamond shows the mean for the group of data. The circles show significant.differences; those that do not intersect, or for which the angle intersection is less than $90^{\circ}$, are significantly different. 
Table 1. Metals values for sediment samples (mg/L), 1989 to 1992.

\begin{tabular}{|c|c|c|c|c|c|c|c|c|c|c|c|c|c|c|c|}
\hline & $\mathrm{Ba}$ & $\mathrm{Cd}$ & Co & Total $\mathrm{Cr}$ & $\mathrm{Cu}$ & $\mathrm{Ni}$ & $\mathrm{Pb}$ & V & Zn & As & $\mathrm{Se}$ & $\mathrm{Hg}$ & $\mathrm{Ag}$ & Mo & $\mathrm{Sb}$ \\
\hline $\begin{array}{l}\text { No. of detections } \\
\text { Maximum value } \\
\text { detected }\end{array}$ & $\begin{array}{r}124 \\
17\end{array}$ & $\begin{array}{r}45 \\
0.41\end{array}$ & $\begin{array}{r}124 \\
0.79\end{array}$ & $\begin{array}{r}101 \\
0.37\end{array}$ & $\begin{array}{l}102 \\
3.8\end{array}$ & $\begin{array}{r}124 \\
1.7\end{array}$ & $\begin{array}{l}54 \\
79\end{array}$ & $\begin{array}{c}120 \\
1.1\end{array}$ & $\begin{array}{r}120 \\
26\end{array}$ & $\begin{array}{l}119 \\
0.47\end{array}$ & $0.02^{2}$ & $\begin{array}{r}50 \\
0.058\end{array}$ & $\begin{array}{r}7 \\
0.06\end{array}$ & 0.09 & $\begin{array}{r}7 \\
0.15\end{array}$ \\
\hline $\begin{array}{l}\text { Minimum value } \\
\text { detected }\end{array}$ & 2.3 & 0.04 & 0.11 & 0.05 & 0.05 & 0.2 & 0.2 & 0.1 & 0.03 & 0.02 & 0.02 & 0.002 & 0.02 & 0.08 & 0.06 \\
\hline $\begin{array}{l}\text { Average } \\
\text { detected value }\end{array}$ & 8.08 & 0.065 & 0.40 & 0.10 & 0.28 & 0.56 & 2.0 & 0.31 & 1.2 & 0.043 & 0.02 & 0.004 & 0.036 & 0.087 & 0.10 \\
\hline $\begin{array}{l}\text { Limit for disposal } \\
\text { of hazardous } \\
\text { waste }\end{array}$ & 100 & 0.75 & 80 & 5 & 25 & 20 & 5 & 24 & 250 & 5 & 1 & 0.2 & 5 & 350 & 15 \\
\hline $\begin{array}{l}\text { Maximum value } \\
\text { as a percent of } \\
\text { disposal limit }\end{array}$ & 17 & 54.7 & 0.99 & 7.4 & 15.2 & 8.5 & 156 & 4.6 & 10.4 & 9.4 & 2 & 29 & 1.2 & 0.03 & 1 \\
\hline
\end{tabular}

Note: Values for 1988 are not included because a different analytical method was used. 
Table 2. Organic compounds and pesticides values for sediment samples, 1988 to 1992.

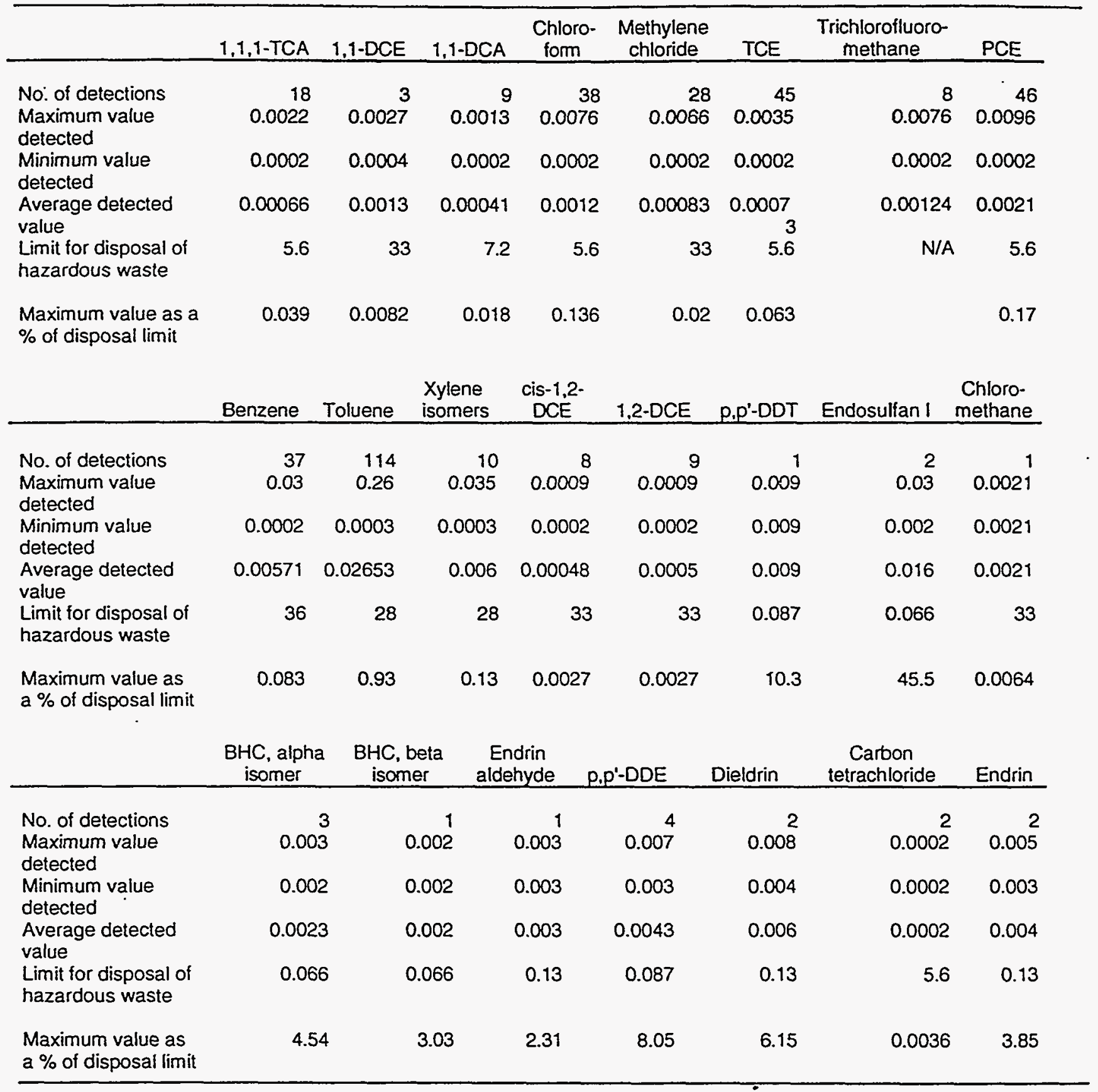


Table 3. Comparison of soils and sediment radionuclide data, 1988 to 1992.

\begin{tabular}{|c|c|c|c|c|c|c|}
\hline Analyte & Mean & Sid error & Mean & $\frac{\text { Soils }}{\text { Std error }}$ & $\begin{array}{l}\text { ANOVA } \\
\text { Prob > F }\end{array}$ & $\begin{array}{l}\text { Significantly } \\
\text { different }\end{array}$ \\
\hline${ }^{239} \mathrm{pu}(\mathrm{Bq} / \mathrm{L})$ & 0.000095 & 0.00002 & 0.000246 & 0.00004 & 0.0010 & Yes \\
\hline${ }^{137} \mathrm{Cs}(\mathrm{Bg} / \mathrm{L})$ & 0.000662 & 0.00018 & 0.0041 & 0.00028 & 0.0000 & Yes \\
\hline $40_{K}(\mathrm{~Bq} / \mathrm{L})$ & 0.492 & 0.007 & 0.479 & 0.010 & 0.2879 & No \\
\hline 235 U $(\mu \mathrm{g} / \mathrm{g})$ & 0.0212 & 0.0007 & 0.0203 & 0.0011 & 0.4399 & No \\
\hline $238 \cup(\mu \mathrm{g} / \mathrm{g})$ & 2.44 & 0.126 & 2.56 & 0.196 & 0.6065 & No \\
\hline${ }^{232}$ Th $(\mu g / g)$ & 6.91 & 0.123 & 6.87 & 0.191 & 0.8557 & No \\
\hline $\begin{array}{l}\text { 226Ra (Bq/L) } \\
228 R a(B q / L)\end{array}$ & $\begin{array}{l}0.0232 \\
0.0252\end{array}$ & $\begin{array}{l}0.0005 \\
0.00055\end{array}$ & $\begin{array}{l}0.238 \\
0.0242\end{array}$ & $\begin{array}{l}0.0008 \\
0.00085\end{array}$ & $\begin{array}{l}0.4928 \\
0.3600\end{array}$ & $\begin{array}{l}\text { No } \\
\text { No }\end{array}$ \\
\hline
\end{tabular}

*Logarithms of $239 \mathrm{Pu}$ were also significantly different.

Table 4. California waste extraction analysis metals from 1991 sediments study (mg/L).

\begin{tabular}{|c|c|c|c|c|c|c|c|}
\hline & Barium & Cadmium & Copper & Lead & Nickel & Vanadium & Zinc \\
\hline \multicolumn{8}{|l|}{ Catch basins } \\
\hline No. of detections & 11 & 11 & 13 & 16 & 14 & 4 & 14 \\
\hline Maximum value detected & 7.4 & 0.26 & 7 & 63 & 1.7 & 0.65 & 80 \\
\hline Minimum value detected & 3.3 & 0.08 & 0.39 & 0.6 & 0.02 & 0.15 & 8.5 \\
\hline Average detected value & 5.2 & 0.126 & 1.64 & 6.81 & 0.57 & 0.32 & 35 \\
\hline $\begin{array}{l}\text { Limit for disposal of } \\
\text { hazardous waste }\end{array}$ & 100 & 1 & 25 & 5 & 20 & 24 & 250 \\
\hline $\begin{array}{l}\text { Maximum value as a } \\
\text { percent of disposal limit }\end{array}$ & 7.4 & 26 & 28 & 1260 & 8.5 & 2.71 & 32 \\
\hline \multicolumn{8}{|l|}{ Open channels } \\
\hline No. of detections & 7 & 2 & 4 & 6 & 7 & 7 & 3 \\
\hline Maximum value detected & 8.9 & 0.17 & 5.7 & 3.6 & 0.9 & 0.59 & 82 \\
\hline Minimum value detected & 5.2 & 0.05 & 1.2 & 0.3 & 0.6 & 0.24 & 18 \\
\hline Average detected value & 7.59 & 0.11 & 2.68 & 1.13 & 0.71 & 0.36 & 43.3 \\
\hline $\begin{array}{l}\text { Limit for disposal of } \\
\text { hazardous waste }\end{array}$ & 100 & 1 & 25 & 5 & 20 & 24 & 250 \\
\hline $\begin{array}{l}\text { Maximum value as a } \\
\text { percent of disposal limit }\end{array}$ & 8.9 & 17 & 22.8 & 72 & 4.5 & 2.46 & 32.8 \\
\hline
\end{tabular}

Table 5. ICAP metals analysis from 1991 sediments study on liquid samples (mg/L).

\begin{tabular}{|c|c|c|c|c|c|c|c|c|c|c|c|c|c|c|c|}
\hline . & As & $\mathrm{Ba}$ & $\mathrm{Be}$ & $\mathrm{Cd}$ & $\mathrm{Cr}$ & Co & $\mathrm{Cu}$ & $\mathrm{Pb}$ & $\mathrm{Hg}$ & Mo & $\mathrm{Ni}$ & $\mathrm{Se}$ & Th & V & Zn \\
\hline No. of detections & 4 & 4 & 1 & 2 & 3 & 2 & 7 & 3 & 5 & 1 & 2 & 1 & 1 & 2 & 7 \\
\hline $\begin{array}{l}\text { Maximum value } \\
\text { detected }\end{array}$ & 0.043 & 11 & 0.01 & 0.47 & 1.4 & 1.2 & 5.4 & 15 & 0.073 & 8.2 & 1.4 & 0.01 & 0.2 & 1.9 & 62 \\
\hline $\begin{array}{l}\text { Minimum value } \\
\text { detected }\end{array}$ & 0.006 & 0.07 & 0.01 & 0.45 & 0.05 & 0.27 & 0.025 & 0.2 & 0.0009 & 8.2 & 1.2 & 0.01 & 0.2 & 1.1 & 0.24 \\
\hline $\begin{array}{l}\text { Average detected } \\
\text { value }\end{array}$ & 0.016 & 2.9 & 0.01 & 0.46 & 0.95 & 0.74 & 1.58 & 5.8 & 0.034 & 8.2 & 1.3 & 0.01 & 0.2 & 1.5 & 12.2 \\
\hline $\begin{array}{l}\text { Limit for disposal of } \\
\text { hazardous waste }\end{array}$ & 5 & 100 & 0.75 & 1 & 5 & 80 & 25 & 5 & 0.2 & 350 & 20 & 1 & 7 & 24 & 250 \\
\hline $\begin{array}{l}\text { Maximum value as a } \\
\% \text { of disposal limit }\end{array}$ & 0.86 & 11 & 1.33 & 47 & 28 & 1.5 & 21.6 & 300 & 36.5 & 2.34 & 7 & & 2.86 & 7.92 & 24.8 \\
\hline
\end{tabular}


Table 6. ICAP metals analysis from 1991 sediments study on solid samples (mg/kg).

\begin{tabular}{|c|c|c|c|c|c|c|c|c|c|c|c|c|c|c|c|c|}
\hline & Sb & As & $\mathrm{Ba}$ & $\mathrm{Be}$ & $\mathrm{Cd}$ & $\mathrm{Gr}$ & Co & $\mathrm{Cu}$ & $\mathrm{Pb}$ & $\mathrm{Hg}$ & Mo & $\mathrm{Ni}$ & $\mathrm{Ag}$ & Th & V & Zn \\
\hline \multicolumn{17}{|l|}{ Background } \\
\hline No. of detections & 0 & 7 & 8 & 7 & 8 & 8 & 8 & 8 & 7 & 3 & 1 & 8 & & & 8 & 8 \\
\hline $\begin{array}{l}\text { Maximum value } \\
\text { detected }\end{array}$ & & 3.8 & 520 & 0.6 & 15 & 75 & 15 & 160 & 320 & 0.14 & 9 & 110 & & & 77 & 290 \\
\hline $\begin{array}{l}\text { Minimum value } \\
\text { detected }\end{array}$ & & 1.4 & 130 & 0.2 & 6 & 18 & 5 & 15 & 6 & 0.05 & 9 & 16 & & & 28 & 27 \\
\hline $\begin{array}{l}\text { Average } \\
\text { detected value }\end{array}$ & & 2.3 & 258 & 0.4 & 9.75 & 39.8 & 9.25 & 40.1 & 56.3 & 0.103 & 9 & 43.5 & & & 43.8 & 78.1 \\
\hline $\begin{array}{l}\text { Limit for disposal } \\
\text { of hazardous } \\
\text { waste }\end{array}$ & 500 & 500 & 10000 & 75 & 100 & 2500 & 8000 & 2500 & 1000 & 20 & 3500 & 2000 & 500 & 700 & 2400 & 5000 \\
\hline $\begin{array}{l}\text { Maximum value } \\
\text { as a percent of } \\
\text { disposal limit }\end{array}$ & & 0.76 & 5.2 & 0.8 & 15 & 3 & 0.19 & 6.4 & 32 & 0.7 & 0.26 & 5.5 & & & 3.2 & 5.8 \\
\hline \multicolumn{17}{|l|}{ Catch basin } \\
\hline No. of detections & 3 & 39 & 41 & 24 & 42 & 42 & 42 & 42 & 42 & 29 & 6 & 42 & 7 & 5 & 42 & 42 \\
\hline $\begin{array}{l}\text { Maximum value } \\
\text { detected }\end{array}$ & 120 & 43 & 250 & 0.3 & 22 & 340 & 54 & 480 & 25000 & 38 & 15 & 200 & 21 & 7 & & 3000 \\
\hline $\begin{array}{l}\text { Minimum value } \\
\text { detected }\end{array}$ & 4 & 0.05 & 50 & 0.2 & 3 & 14 & 3 & 2.3 & 4.8 & 0.05 & 4 & 15 & 1 & 2 & 9 & 65 \\
\hline $\begin{array}{l}\text { Average } \\
\text { detected value }\end{array}$ & 43 & 4.28 & 113.4 & 0.22 & 7.14 & 49.0 & 9.57 & 67.5 & 676.5 & 1.75 & 7.67 & 47.1 & 7 & 4 & 23.5 & 420 \\
\hline $\begin{array}{l}\text { Limit for disposal } \\
\text { of hazardous } \\
\text { waste }\end{array}$ & 500 & 500 & 10000 & 75 & 100 & 2500 & 8000 & 2500 & 1000 & 20 & 3500 & 2000 & 500 & 700 & 2400 & 5000 \\
\hline $\begin{array}{l}\text { Maximum value } \\
\text { as a percent of } \\
\text { disposal limit }\end{array}$ & 24 & 8.6 & 2.5 & 0.4 & 22 & $\begin{array}{l}13.6 \\
\cdot\end{array}$ & 0.68 & 19.2 & 2500 & 190 & 0.43 & 10 & 4.2 & 1 & 2.54 & 60 \\
\hline \multicolumn{17}{|l|}{ Open channel } \\
\hline No. of detections & 2 & 16 & 18 & 16 & 27 & 27 & 27 & 27 & 25 & 8 & 0 & 27 & 0 & 3 & 27 & $7 \quad 27$ \\
\hline $\begin{array}{l}\text { Maximum value } \\
\text { detected }\end{array}$ & 5 & 7.3 & 330 & 0.7 & 13 & 66 & 14 & 54 & 80 & 1.2 & 0 & 70 & 0 & 6 & 54 & 41500 \\
\hline $\begin{array}{l}\text { Minimum value } \\
\text { detected }\end{array}$ & 4 & 0.06 & 38 & 0.2 & 2 & 15 & 2 & 7 & 4 & 0.06 & 0 & 13 & 0 & 4 & 9 & 34 \\
\hline $\begin{array}{l}\text { Average } \\
\text { detected value }\end{array}$ & 4.5 & 3.1 & 172 & 0.35 & 7.77 & 31.5 & 9.05 & 22.7 & 15.2 & 0.393 & & 32 & & 4.67 & 32.8 & 165 \\
\hline $\begin{array}{l}\text { Limit for disposal } \\
\text { of hazardous } \\
\text { waste }\end{array}$ & 500 & 500 & 10000 & 75 & 100 & 2500 & 8000 & 2500 & 1000 & 20 & 3500 & 2000 & 500 & 700 & 2400 & 5000 \\
\hline $\begin{array}{l}\text { Maximum value } \\
\text { as \% of disposal } \\
\text { limit }\end{array}$ & 1 & 1.46 & 3.3 & 0.93 & 13 & 2.64 & 0.18 & 2.16 & 8 & 6 & 0 & 3.5 & & 0.86 & 2.25 & 30 \\
\hline
\end{tabular}


Table 7. Radionuclide analysis for 1991 sediment study (pCi/g, unless otherwise noted).

\begin{tabular}{|c|c|c|c|c|c|c|c|c|c|}
\hline Locations & $\begin{array}{l}\text { Gross } \\
\text { alpha } \\
\end{array}$ & $\begin{array}{c}\text { Gross } \\
\text { beta }\end{array}$ & $\begin{array}{c}3 \mathrm{H} \\
(\mathrm{pCil}) \\
\end{array}$ & $\begin{array}{c}\% \\
\text { moisture }\end{array}$ & $40 \mathrm{~K}$ & ${ }^{137} \mathrm{Cs}$ & ${ }^{226} \mathrm{Ra}$ & ${ }^{228} \mathrm{Th}$ & ${ }^{232} \mathrm{Th}$ \\
\hline \multicolumn{10}{|l|}{ Background } \\
\hline $\begin{array}{l}\text { No. of } \\
\text { detections }\end{array}$ & 4 & 8 & 5 & 8 & 8 & 0 & .7 & 7 & 6 \\
\hline $\begin{array}{l}\text { Maximum value } \\
\text { detected }\end{array}$ & 8 & 19 & 1302 & 8.8 & 18.99 & & 0.9029 & 1.114 & 1.121 \\
\hline $\begin{array}{l}\text { Minimum value } \\
\text { detected }\end{array}$ & 4 & 13 & 245 & 1.5 & 8.097 & & 0.3623 & 0.5109 & 0.5342 \\
\hline $\begin{array}{l}\text { Average } \\
\text { detected value }\end{array}$ & 6.5 & 15.6 & 711 & 4.3 & 13.59 & & 0.5612 & 0.8294 & 0.8099 \\
\hline \multicolumn{10}{|l|}{ Catch basin } \\
\hline $\begin{array}{l}\text { No. of } \\
\text { detections }\end{array}$ & 37 & 43 & 41 & 34 & 45 & 8 & 29 & 39 & 24 \\
\hline $\begin{array}{l}\text { Maximum value } \\
\text { detected }\end{array}$ & 11 & 20 & 2719 & 54.2 & 528 & 0.7091 & 0.6985 & 1.226 & 3.681 \\
\hline $\begin{array}{l}\text { Minimum value } \\
\text { detected }\end{array}$ & 0.5 & 5 & 12 & 0.4 & 0.4453 & 0.09741 & 0.1961 & 0.05515 & 0.3531 \\
\hline $\begin{array}{l}\text { Average } \\
\text { detected value }\end{array}$ & 3.4 & 13 & 654 & 13.5 & 20.72 & 0.3256 & 0.3870 & 0.6029 & 0.8641 \\
\hline \multicolumn{10}{|l|}{ Open channel } \\
\hline $\begin{array}{l}\text { No. of } \\
\text { detections }\end{array}$ & 26 & 28 & 28 & 27 & 28 & 9 & 25 & 26 & 24 \\
\hline $\begin{array}{l}\text { Maximum value } \\
\text { detected }\end{array}$ & 10 & 29 & 6182 & 49.8 & 105 & 0.726 & 1.407 & 7.122 & 3.33 \\
\hline $\begin{array}{l}\text { Minimum value } \\
\text { detected }\end{array}$ & 0.5 & 10 & 1.5 & 0.7 & 0.642 & 0.0724 & 0.1627 & 0.468 & 0.1235 \\
\hline $\begin{array}{l}\text { Average } \\
\text { detected value }\end{array}$ & 4.8 & 16 & 782 & 9.0 & 16.8 & 0.2264 & 0.5823 & 1.057 & 0.8641 \\
\hline \multicolumn{10}{|l|}{ Liguid (mg/L) } \\
\hline $\begin{array}{l}\text { No. of } \\
\text { detections }\end{array}$ & 5 & 6 & 6 & 0 & 2 & 1 & 1 & 2 & 1 \\
\hline $\begin{array}{l}\text { Maximum value } \\
\text { detected }\end{array}$ & 18 & 25 & 6268 & . & 11.62 & 0.4909 & 0.4984 & 0.9726 & 0.5581 \\
\hline $\begin{array}{l}\text { Minimum value } \\
\text { detected }\end{array}$ & 0.5 & 10 & 236 & & 9.173 & 0.4909 & 0.4984 & 0.6237 & 0.5581 \\
\hline $\begin{array}{l}\text { Average } \\
\text { detected value }\end{array}$ & 5.6 & 15 & 1737 & & 10.3965 & 0.4909 & 0.4984 & 0.79815 & 0.5581 \\
\hline
\end{tabular}


Table 8 . Volatile organic compounds in 1991 study detected by EPA method 8270 and for which there are disposal limits, $\mathrm{mg} / \mathrm{kg}$ unless otherwise noted.

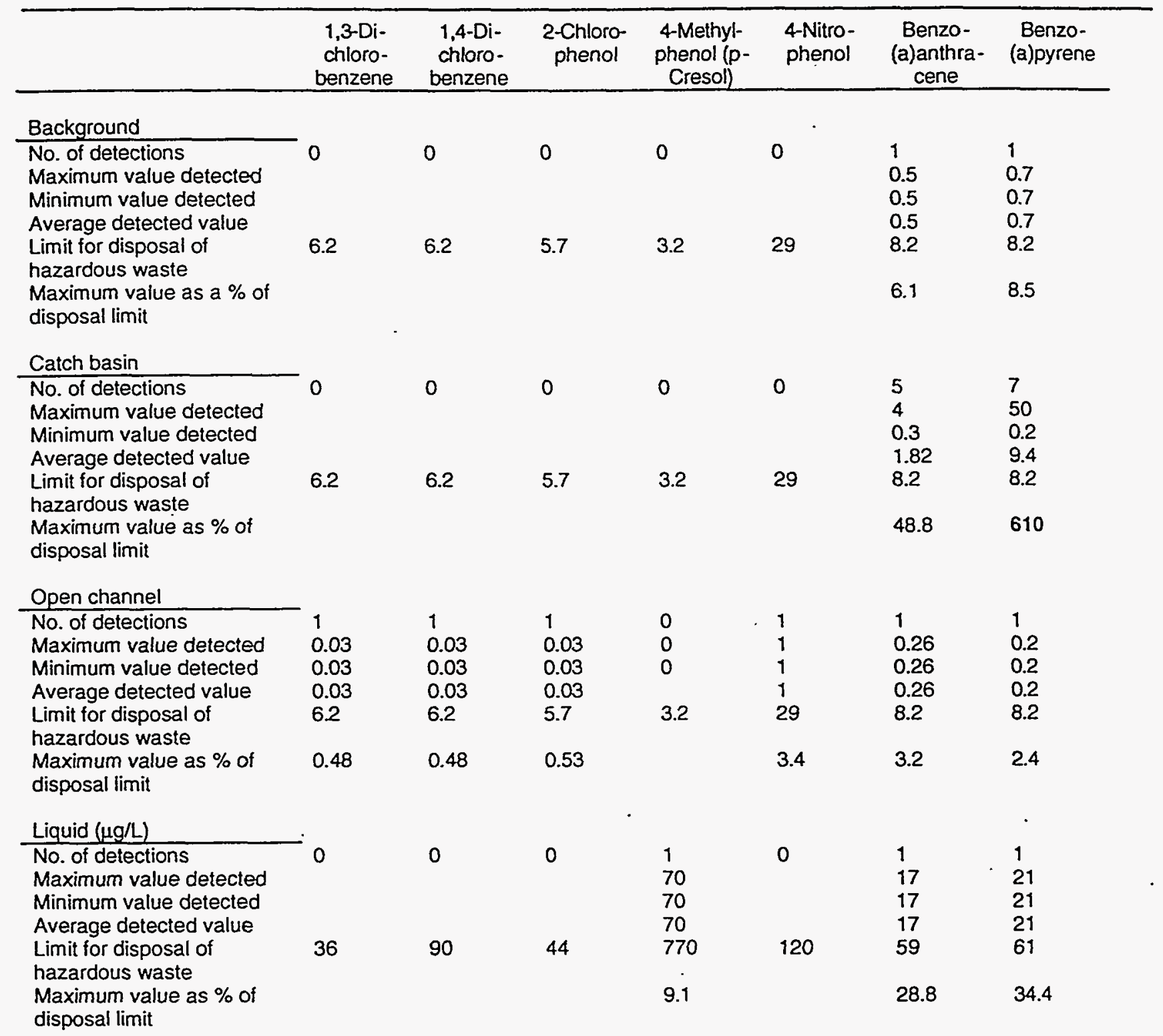


Table 8. Volatile organic compounds in 1991 study detected by EPA method 8270 and for which there are disposal limits, $\mathrm{mg} / \mathrm{kg}$ unless otherwise noted. (continued)

\begin{tabular}{|c|c|c|c|c|c|c|}
\hline - & Chrysene & $\begin{array}{l}\text { Dibenzo- } \\
\text { (a,h)an- } \\
\text { thracene }\end{array}$ & $\begin{array}{l}\text { Fluor- } \\
\text { anthene }\end{array}$ & $\begin{array}{c}\text { Indeno(1,2, } \\
3-c, d)- \\
\text { pyrene } \\
\end{array}$ & Phenol & $\begin{array}{c}\text { Bis-(2- } \\
\text { chloro- } \\
\text { ethyl)ether }\end{array}$ \\
\hline \multicolumn{7}{|l|}{ Background } \\
\hline No. of detections & 1 & 0 & 2 & 0 & 0 & 0 \\
\hline Maximum value detected & 0.6 & & 1.1 & & & \\
\hline Minimum value detected & 0.6 & & 0.6 & & & \\
\hline Average detected value & 0.6 & & 0.85 & & & \\
\hline $\begin{array}{l}\text { Limit for disposal of } \\
\text { hazardous waste }\end{array}$ & 8.2 & 8.2 & 8.2 & 8.2 & 6.2 & 7.2 \\
\hline $\begin{array}{l}\text { Maximum value as \% of } \\
\text { disposal limit }\end{array}$ & 7.3 & & 13.4 & & & \\
\hline \multicolumn{7}{|l|}{ Catch basin } \\
\hline No. of detections & 6 & 0 & 8 & 2 & 0 & 0 \\
\hline Maximum value detected & 4.6 & & 8 & 1.6 & & \\
\hline Minimum value detected & 0.3 & & 0.3 & 0.5 & & \\
\hline Average detected value & 2.3 & & 2.9 & 1.1 & & \\
\hline $\begin{array}{l}\text { Limit for disposal of } \\
\text { hazardous waste }\end{array}$ & 8.2 & 8.2 & 8.2 & 8.2 & 6.2 & 7.2 \\
\hline $\begin{array}{l}\text { Maximum value as \% of } \\
\text { disposal limit }\end{array}$ & 56.1 & & 97.6 & 19.5 & & \\
\hline \multicolumn{7}{|l|}{ Open channel } \\
\hline No. of detections & 3 & 1 & 3 & 1 & 1 & 1 \\
\hline Maximum value detected & 0.3 & 0.22 & 0.54 & 0.2 & 0.03 & 0.03 \\
\hline Minimum value detected & 0.09 & 0.22 & 0.09 & 0.2 & 0.03 & 0.03 \\
\hline Average delected value & 0.20 & 0.22 & 0.28 & 0.2 & 0.03 & 0.03 \\
\hline $\begin{array}{l}\text { Limit for disposal of } \\
\text { hazardous waste }\end{array}$ & 8.2 & 8.2 & 8.2 & 8.2 & 6.2 & 7.2 \\
\hline $\begin{array}{l}\text { Maximum value as \% of } \\
\text { disposal limit }\end{array}$ & 3.66 & 2.68 & 6.59 & 2.44 & 0.48 & 0.42 \\
\hline \multicolumn{7}{|l|}{ Liguid $(\mu \mathrm{g} / \mathrm{L})$} \\
\hline No. of detections & 1 & 1 & 2 & 0 & 0 & 0 \\
\hline Maximum value detected & 17 & 12 & 27 & & & \\
\hline Minimum value detected & 17 & 12 & 20 & & & \\
\hline Average detected value & 17 & 12 & 23.5 & & & \\
\hline $\begin{array}{l}\text { Limit for disposal of } \\
\text { hazardous waste }\end{array}$ & 59 & 55 & 68 & 5.5 & 39 & 33 \\
\hline $\begin{array}{l}\text { Maximum value as } \% \text { of } \\
\text { disposal limit }\end{array}$ & 28.8 & 21.8 & 39.7 & & & \\
\hline
\end{tabular}


Table 9. Pesticides detected in 1991 study in $\mathrm{mg} / \mathrm{kg}$ unless otherwise noted.

Dieldrin P.p'-DDE p.p'-DDT Endo- $\begin{gathered}\text { Endo- } \\ \text { sulfan I sulfan II }\end{gathered}$

Background

\begin{tabular}{|c|c|c|c|c|c|c|c|c|c|c|}
\hline No. of detections & 1 & 1 & 0 & 0 & 0 & 0 & 0 & 0 & 0 & 0 \\
\hline Value detected & 0.14 & 0.006 & & & & & & & & \\
\hline $\begin{array}{l}\text { Limit for disposal of } \\
\text { hazardous waste } \\
\text { Maximum value as } \\
\% \text { of disposal limit }\end{array}$ & $\begin{array}{l}0.13 \\
108\end{array}$ & $\begin{array}{r}0.087 \\
6.9\end{array}$ & 0.087 & 0.066 & 0.13 & 0.13 & 0.066 & 0.18 & 0.066 & 0.066 \\
\hline \multicolumn{11}{|l|}{ Catch basin } \\
\hline No. of detections & 2 & 0 & 3 & 0 & 2 & 0 & 1 & 2 & 1 & 0 \\
\hline $\begin{array}{l}\text { Maximum value } \\
\text { detected }\end{array}$ & 0.07 & & 0.19 & & 0.12 & & 0.04 & 0.3 & 0.006 & \\
\hline $\begin{array}{l}\text { Minimum value } \\
\text { detected }\end{array}$ & 0.02 & & 0.02 & & 0.01 & & 0.04 & 0.1 & 0.006 & \\
\hline $\begin{array}{l}\text { Average detected } \\
\text { value }\end{array}$ & 0.045 & & 0.10267 & & 0.065 & & 0.04 & 0.2 & 0.006 & \\
\hline $\begin{array}{l}\text { Limit for disposal of } \\
\text { hazardous waste }\end{array}$ & 0.13 & 0.087 & 0.087 & 0.066 & 0.13 & 0.13 & 0.066 & 0.18 & 0.066 & 0.066 \\
\hline $\begin{array}{l}\text { Maximum value as } \\
\% \text { of disposal limit }\end{array}$ & 53.8 & & 218 & & 92.3 & & 60.6 & 167 & 9.1 & \\
\hline \multicolumn{11}{|l|}{ Open channel } \\
\hline No. of detections & 0 & 0 & 0 & 1 & 0 & 1 & 0 & 0 & 1 & 0 \\
\hline Value detected & & & & 0.17 & & 0.43 & & & 0.006 & \\
\hline $\begin{array}{l}\text { Limit for disposal of } \\
\text { hazardous waste } \\
\text { Maximum value as } \\
\% \text { of disposal limit }\end{array}$ & 0.13 & 0.087 & 0.087 & $\begin{array}{r}0.066 \\
258\end{array}$ & 0.13 & $\begin{array}{l}0.13 \\
331\end{array}$ & 0.066 & 0.18 & $\begin{array}{r}0.066 \\
9.1\end{array}$ & 0.066 \\
\hline \multicolumn{11}{|l|}{ Liquid $(\mu g / L)$} \\
\hline No. of detections & 0 & 0 & 0 & 0 & 0 & 0 & 0 & 0 & 0 & 1 \\
\hline Value detected & & & & & & & & & & 0.09 \\
\hline $\begin{array}{l}\text { Limit for disposal of } \\
\text { hazardous waste } \\
\text { Maximum value as } \\
\% \text { of disposal limit }\end{array}$ & 17 & 31 & 3.9 & 23 & 29 & 29 & 16 & 250 & 0.14 & $\begin{array}{r}23 \\
0.39\end{array}$ \\
\hline
\end{tabular}


Table 10. Volatile organic compounds detected in 1991 study (EPA method 5030/8240) for which there are disposal limits, $\mathrm{mg} / \mathrm{kg}$ unless otherwise noted.

$\begin{aligned} & \text { 1,2-Di- Ace- } \\ & \text { chloro- } \\ & \text { ethene }\end{aligned}$

Background

No. of detections

Maximum value

detected

Minimum value

detected

Average detected

value

Limit for disposal of

hazardous waste

Maximum value as

$\%$ of disposal limit

Catch basin

No. of detections
Maximum value
detected
Minimum value
detected
Average detected
value
Limit for disposal of
hazardous waste
Maximum value as
$\%$ of disposal limit

$\begin{array}{rrrrrrrrrrrr}1 & 1 & 0 & 0 & 1 & 1 & 7 & 6 & 6 & 1 & 2 \\ 0.03 & 0.067 & & & 0.005 & 0.005 & 0.35 & 0.27 & 0.089 & 0.03 & 280 \\ 0.03 & 0.067 & & & 0.005 & 0.005 & 0.009 & 0.007 & 0.005 & 0.03 & 0.011 \\ 0.03 & 0.067 & & & 0.005 & 0.005 & 0.0819 & 0.089 & 0.031 & 0.03 & 140 \\ 33 & 160 & 5.6 & 5.6 & 5.6 & 33 & 28 & 5.6 & 28 & 33 & 33 \\ 0.091 & 0.042 & & & 0.089 & 0.015 & 1.25 & 4.82 & 0.32 & 0.091 & 848\end{array}$

Open channel

No. of detections

Maximum value

detected

Minimum value

detected

Average detected

value

Limit for disposal of

hazardous waste

Maximum value as

$\%$ of disposal limit

$\begin{array}{llll}0 & 0 & 0\end{array}$

$\begin{array}{llllll}33 & 160 & 5.6 & 5.6 & 5.6 & 33\end{array}$

$\begin{array}{rrr}5 & 0 & 2 \\ 0.03 & & 0.039\end{array}$

$0.005 \quad 0.008$

$0.0112 \quad 0.0235$

$0.11 \quad 0.14$

Liquid $(\mu g / 2)$

No. of detections

Maximum value

detected

Minimum value

detected

Average detected

value

Limit for disposal of hazardous waste

Maximum value as

$\%$ of disposal limit

\begin{tabular}{|c|c|c|c|c|c|c|c|c|c|c|}
\hline \multirow[t]{4}{*}{0} & 1 & 1 & 0 & 0 & 0 & 2 & 0 & 6 & 0 & 0 \\
\hline & 0.1 & 0.023 & & & & 0.038 & & 0.026 & & \\
\hline & 0.1 & 0.023 & & & & 0.016 & & 0.008 & & \\
\hline & 0.1 & 0.023 & & & & 0.027 & & 0.0175 & & \\
\hline \multirow[t]{6}{*}{33} & 160 & 5.6 & 5.6 & 5.6 & 33 & 28 & 5.6 & 28 & 33 & 33 \\
\hline & 0.063 & 0.41 & & & & 0.14 & & 0.093 & & \\
\hline & 3 & 3 & 1 & 1 & 1 & 2 & 1 & 1 & 1 & 0 \\
\hline & 26 & 5.7 & 32 & 0.7 & 1.5 & 67 & 4.9 & 2.1 & 0.9 & \\
\hline & 0.11 & 1.3 & 32 & 0.7 & 1.5 & 0.036 & 4.9 & 2.1 & 0.9 & \\
\hline & 13.0 & 2.83 & 32 & 0.7 & 1.5 & 33.5 & 4.9 & 2.1 & 0.9 & \\
\hline \multirow[t]{2}{*}{54} & 280 & 46 & 54 & 54 & 20 & 80 & 56 & 320 & 54 & 270 \\
\hline & 9.3 & 12.3 & 59.3 & 1.3 & 7.5 & 83.8 & 8.75 & 0.66 & 1.67 & \\
\hline
\end{tabular}


Table 11. Summary of materials found in the sediment studies above hazardous waste disposal limits.

\begin{tabular}{|c|c|c|c|c|}
\hline Study & Contaminant & Waste limit & Value detected & Location \\
\hline $\begin{array}{l}\text { Surveillance } \\
1989 \text { Study } \\
1991 \text { Study }\end{array}$ & $\begin{array}{l}\text { Lead } \\
\text { Lead } \\
\text { Lead } \\
\text { Mercury } \\
\text { Benzo(a)-pyrene } \\
\text { Dieldrin } \\
\text { p.p'-DDT } \\
\text { Endosulfan I } \\
\text { Endosulfan sulfate } \\
\text { Vinyl chloride }\end{array}$ & $\begin{array}{l}5 \mathrm{mg} / \mathrm{L} \\
5 \mathrm{mg} / \mathrm{L} \\
5 \mathrm{mg} / \mathrm{L} \\
5 \mathrm{mg} / \mathrm{L} \\
5 \mathrm{mg} / \mathrm{L} \\
20 \mathrm{mg} / \mathrm{L} \\
8.2 \mathrm{mg} / \mathrm{kg} \\
0.14 \mathrm{mg} / \mathrm{kg} \\
0.087 \mathrm{mg} / \mathrm{kg} \\
0.087 \mathrm{mg} / \mathrm{kg} \\
0.066 \mathrm{mg} / \mathrm{kg} \\
0.13 \mathrm{mg} / \mathrm{kg} \\
33 \mathrm{mg} / \mathrm{kg}\end{array}$ & $\begin{array}{l}79 \mathrm{mg} / \mathrm{L} \\
18 \mathrm{mg} / \mathrm{L} \\
14 \mathrm{mg} / \mathrm{L} \\
15 \mathrm{mg} / \mathrm{L} \\
25000 \mathrm{mg} / \mathrm{L} \\
38 \mathrm{mg} / \mathrm{L} \\
50 \mathrm{mg} / \mathrm{kg} \\
0.13 \mathrm{mg} / \mathrm{kg} \\
0.19 \mathrm{mg} / \mathrm{kg} \\
0.098 \mathrm{mg} / \mathrm{kg} \\
0.17 \mathrm{mg} / \mathrm{kg} \\
0.43 \mathrm{mg} / \mathrm{kg} \\
280 \mathrm{mg} / \mathrm{kg}\end{array}$ & $\begin{array}{l}\text { ASE } \\
\text { B438 } \\
\text { Duplicate at B } 438 \\
\text { CB-190T-49 } \\
\text { CB-280D-24 } \\
\text { CB-320F-17 } \\
\text { CB-320F-17 } \\
\text { BS-6-6 } \\
\text { CB-410E-044 } \\
\text { CB-190Q-10 1/2 } \\
\text { OCS-460-1-6 } \\
\text { OCS-150-1 } \\
\text { CB-320F-17 }\end{array}$ \\
\hline
\end{tabular}




\section{References}

Brekke, D. D., R. C. Holland, M. G. Brown, and T. M. Carlsen (1989), Environmental Monitoring at the Lawrence Livermore National Laboratory, 1988 Annual Report, Lawrence Livermore National Laboratory, Livermore, CA (UCRL-50027-88).

Gallegos, G. M., B. K. Balke, K. A. Surano, W. G. Hoppes, P. J. Tate, J. C. Steenhoven, B. C. Fields, L. M. Garcia, and K. C. Lamson (1992), Environmental Report for 1991, Lawrence Livermore National Laboratory, Livermore, CA (UCRL-50027-91).

Gallegos, G. M., S. M. Wander, B. K. Balke, E. Christofferson, P. J. Tate, K. A. Surano, R. J. Harrach, L. M. Garcia, B. C. Fields, W. G. Hoppes, and R. A. Failor (1993), Environmental Report for 1992, Lawrence Livermore National Laboratory, Livermore, CA (UCRL-50027-92).

Holland, R. (1993), SAIC, Inc., Pleasanton, CA, personal communication.

Sims, J. M., K. A. Surano, K. C. Lamson, and M. G. Brown (1990), Environmental Report for 1989, Lawrence Livermore National Laboratory, Livermore, CA (UCRL-5002789).

Sims, J. M.; K. A. Surano, K. C. Lamson, B. K. Balke, and J. C. Steenhoven (1991), Environmental Report for 1990, Lawrence Livermore National Laboratory, Livermore, CA (UCRL-50027-90).

Thorpe, R. K., W. F. Isherwood, M. D. Dresen, and C.P. Webster-Scholten (1990), CERCLA Remedial Investigation Report for the LLNL Livermore Site, Lawrence Livermore National Laboratory, Livermore, CA (UCAR-10299, Volumes 1 to 5).

U.S. Department of Energy (1991), Environmental Regulatory Guide for Radiological Effluent Monitoring and Environmental Surveillance, U.S. Department of Energy, Washington, DC (DOE/EH-0173T). 
Appendix A

Letter Report for 1989 Sediment Study 
$\because$ :IatStation L. . ‘.528

May 16, 1989

Ext:

To:

Connie Degrange

From: $\quad$ F. Hoffman

Re: $\quad$ Storm Drain Remediation Issues

As a result of the LLNL Ground Water Project Baseline Public Health Assessment (BPHA) a series of samples were collected from storm drain sedimenits, suifface.waters, and surface - sectiments. See the attached summary of Baseline Public Health Assessment Site Sampling. -protocols and sampling location map. We have provided copies of the resutitu of thesé : sampling and analyses to Robert Holland and Robert Fisher of your division. The results. of several samples taken from the south west portion of the Laboratory are notable Below: I have provided the results of the analyses of thèse samples and indicated analytes that are - elevated relative to background.

1. Sediments from the storm drain culvert just east of Bldg. 438 were found to have elevated concentrations of the following analytes (this stom drain location was sampled in duplicate):

$\begin{array}{lll}\text { Sample çode: } & \text { SSD-008 } & \text { SSD-009 }\end{array}$

Analyte:

Tritium :

Arochlor. 1254

Lindane

C20-C35 Hydrocarbons

(Burker Oils?)

Leảd (STLC)

${ }^{*}$ (note: the Lead STLC limit for hazardous waste is $5 \mathrm{mg} / \mathrm{l}$ ).

2. A surface water sample collected from the Bldg 438 storm drain was found to contain elevvated concentrations of the following analytes:

Sample code:

SDW-002

Arialyte: $\cdot$.

11,000

1.3

ND

0.088

pCi/

Chromium :-

5,000

$18^{*}$

3,000

$14^{*}$

$0.7 \div \mathrm{mg} / \mathrm{kg}$

$\mathrm{mg} / \mathrm{kg}$

Lead $\quad \because$

PCE

$70^{*}$

Units

Freon 11

15

5

*(note: the drinking wáter limits for Chromium is $50 \mu \mathrm{g} / \mathrm{l}$ ). 


\section{Baseline Public Health Assessment Site Sampling Protocol}

Samples collected for organic compound and radionuclide analyses were collected into steam cleaned 1.5 inch diameter steel core tubes, the ends of the core tubes were covered with clean teflon sheets and sealed with plastic caps and duct tape. Samples were collected at a depth of $5 \mathrm{~cm}$. Areas with fine grain materials were selected for sampling. Leaves, twigs, pieces of plastic, etc. were omitted from the sample. Soil samples collected for metal analyses were collected directly into clean zip-lock baggies. Surface water samples were bailed and transferred to or collected directly into appropriate sample containers. Brown and Caldwell analyzed for the organic compounds and coliform bacteria. Clayton Environmental Consultants analyzed for metals. TMA/ Norcal analyzed for radionuclides. The surface soils in both the arroyos were each sampled in triplicate. Analyses requested for each soil or water sample are summarized in Table 1. The number of samples collected and sample codes used are summarized in Tables 2 and 3, below.

Sampling locations for the Baseline Public Health Assessment Site Sampling are shown in draft Figures 2 and 23, both taken from the Baseline Public Health Assessment, in preparation. Copy of the analytical result sheets for Baseline Public Health Assessment Site Sampling are attached.

Table 1. Analyses requested for each soil or water sample

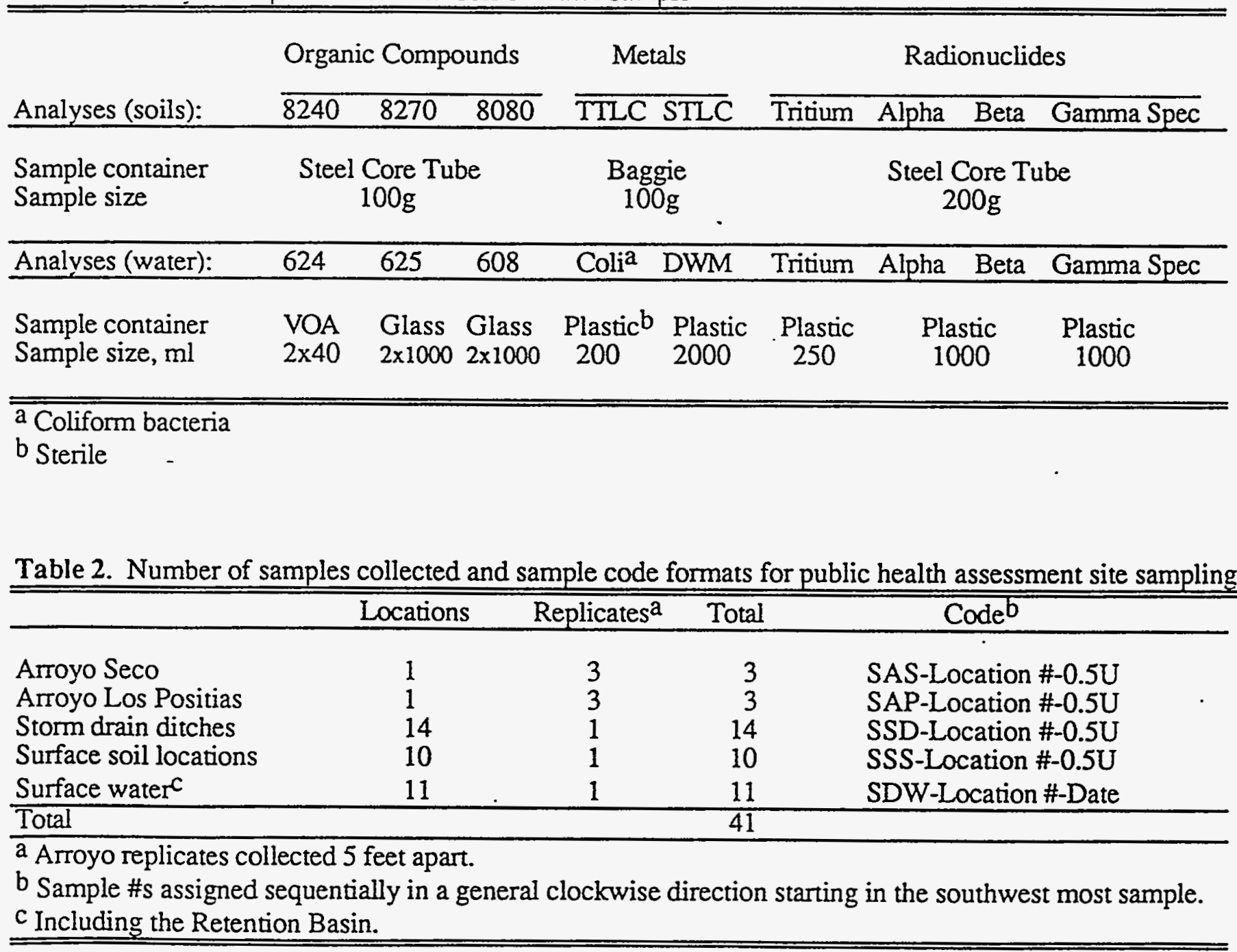


Table 3. Summary of public health assessment site sampling codes

Arroyo Seco Sediments: Collected February 7, 1989.

Location:

Sample Code:

Comments:

SAS-011-0.5u-1

Note: SAS-011 was originally identified as

SAS-001.

2
3 $\quad$ SAS- $011-0.5 \mathrm{u}-2$

Arroyo Las Positias Sediments: Collected February 7, 1989.

Location:

Sample Code:

Comments:

\begin{tabular}{ll}
\hline 1 & SAP-001-0.5u-1 \\
2 & SAP-001-0.5u-2 \\
3 & SAP-001-0.5u-3 \\
\hline
\end{tabular}

Storm Drain Sediments: Collected February 7, 1989 and February 15, 1989.

Location: . Sample Code:

1 SSD-001-0.5u

2 SSD-002-0.5u

3 SSD-003-0.5u

4 SSD-004-0.5u

5 SSD-005-0.5u

6 SSD-006-0.5u

7 SSD-007-0.5u

8 SSD-008-0.5u

9 SSD-009-0.5u

10 SSD-010-0.5u

11 SSD-011-0.5u

12 SSD-012-0.5u

13 SSD-013-0.5u

14
SSD-014-0.5u
Comments:

Sandy silt

Dark brown soft silt

Brown silty clay

Silty sand

Silty sand

Silty sand

Silty sand

Darkly stained fine silt

Replicate of location 8

Silty sand with gravel

Fine silt

Fine silt

Very fine clay (Drilling mud?)

Fine silt

Storm Drain Waters: Collected February 9, 1989.

\begin{tabular}{lll} 
Location: & Sample Code: & Comments: \\
\hline 1 & SDW-001 & \\
2 & SDW-002 & \\
3 & SDW-003 & \\
4 & SDW-004 & \\
5 & SDW-005 & \\
6 & SDW-006 & \\
7 & SDW-007 & DDW Trip blank \\
8 & SDW-008 & Collected in duplicate for RAD only \\
9 & SDW-009 & \\
10 & SDW-010 & \\
11 & SDW-011 & \\
\hline \hline
\end{tabular}


Table 3 (cont.). Summary of public health assessment site sampling codes

Surface sediments: Collected April 11, 1989.

Location: Sample Code:

\begin{tabular}{lll}
\hline 1 & SSS-001 & Fine clat \\
2 & SSS-002 & Near helipad \\
3 & SSS-003 & Next to ball field \\
4 & SSS-004 & Rubble piles \\
5 & SSS-005 & Near LN gas tanks \\
6 & SSS-006 & Behind DWTF \\
7 & SSS-007 & Dirt piles \\
8 & SSS-008 & Old solar ponds \\
9 & SSS-009 & Next B-514 yard \\
10 & SSS-010 & Next to B-518 \\
\hline \hline
\end{tabular}




\section{Appendix B}

\section{Sediment Study Sampling Plan}




\section{LLNL Storm Drain Sampling Plan and Follow-Up Cleanout and Maintenance Proposal}

August 1991

W. W. Schwartz, Project Engineer

Operations and Regulatory Affairs Division 


\section{Contents}

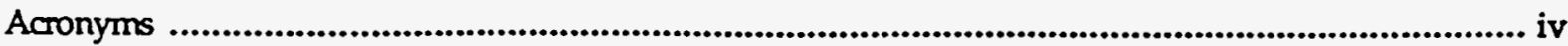

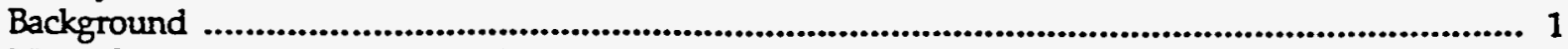

LLNL Storm Drain Grading and Drainage .......................................................................... 1

Storm Drain System at LLNL .................................................................................................. 2

LLNL Storm Drain Sampling Plan ................................................................................................ 2

Purpose for Sampling ............................................................................................................... 2

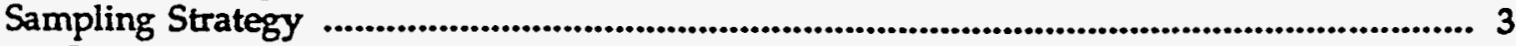

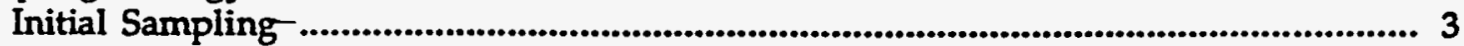

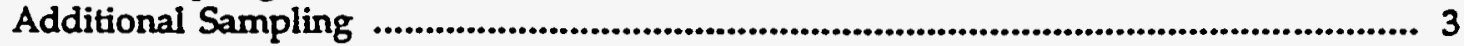

QA Sampling .................................................................................................................. 3

Applicable Regulations and Methods ..................................................................................4

Media to be Sampled and Sample Types ......................................................................... 4

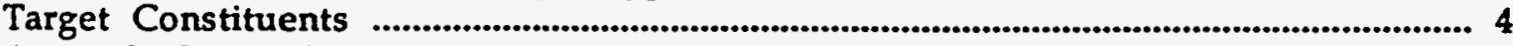

Area to be Sampled .................................................................................................................. 5

Sampling Procedure ........................................................................................................... 5

Quality Assurance/Quality Control ............................................................................. 5

Duplicate Samples ................................................................................... 5

Blank Samples ........................................................................................ 5

Background Samples .................................................................................................... 5

Documentation and Reporting .............................................................................. 5

QA/QC Policy and Procedures ................................................................................. 6

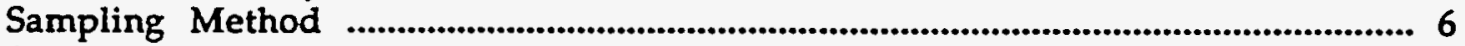

Sample Labeling .............................................................................................................. 7

Sample Transportation, Treatment, and Storage .......................................................... 7

Chain-of-Custody Forms and Field Tracking Forms ................................................................ 7

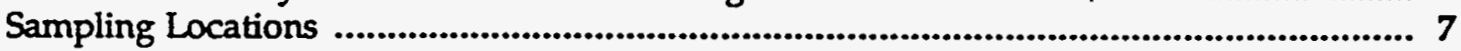

Analytical Laboratories ............................................................................................. 10

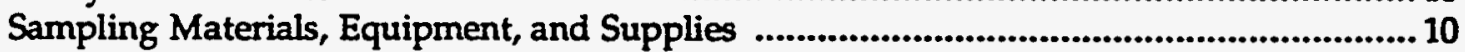

Follow-Up Activities ............................................................................................................... 11

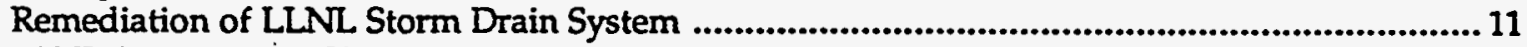

LLNL Storm Drain Cleanout and Disposal Recommendations ................................................. 11

Future and Ongoing Monitoring of the LLNL Storm Drain System ........................................ 11

Documentation of LLNL Storm Drain System .................................................................... 11

Appendix A. LLNL Storm Drain Site Map ..................................................................... A-1

Appendix B. Sampling Location Site Map ................................................................... B-1

Appendix C. Sampling Location Data Sheet ....................................................................... C-1 


\section{Acronyms}

\begin{tabular}{|c|c|}
\hline $\mathrm{ACP}$ & asbestos-impregnated cement pipe \\
\hline Ag & silver \\
\hline As & arsenic \\
\hline $\mathbf{B a}$ & barium \\
\hline BCA & Brown and Caldwell Analytical Laboratory \\
\hline Be & beryllium \\
\hline BF & background field sample \\
\hline BPHA. & Baseline Public Health Assessment \\
\hline BS & background sample \\
\hline CB & catch basin \\
\hline CCR & California Code of Regulations \\
\hline $\mathrm{Cd}$ & cadmium \\
\hline CEC & Clayton Environmental Consultants Analytical Laboratory \\
\hline CFR & Code of Federal Regulations \\
\hline CIP & cast iron pipe \\
\hline CMP & corrugated metal pipe \\
\hline CMPA & corrugated metal pipe-arched \\
\hline Co & cobalt \\
\hline $\mathrm{Cr}$ & chromium \\
\hline Gr & copper \\
\hline DP & duplicate sample \\
\hline EAS & LLNL Environmental-Analytical Sciences Laboratory \\
\hline EPA & U.S. Environmental Protection Agency \\
\hline EPD & LLNL Environmental Protection Department \\
\hline FAX & facsimile \\
\hline FB & field blank \\
\hline FS & field sample \\
\hline $\mathrm{Hg}$ & mercury \\
\hline ID & identification \\
\hline L & liquid \\
\hline LCS & laboratory control standards \\
\hline LLNL & Lawrence Livermore National Laboratory \\
\hline MDL & minimum detection limit \\
\hline MH & manhole \\
\hline Mo & molybdenum \\
\hline $\mathbf{N}$ & no \\
\hline NA & not applicable \\
\hline $\mathbf{N i}$ & nickel \\
\hline $\mathbf{O}$ & other \\
\hline OCS & open channel site \\
\hline Pb & lead \\
\hline PCB & polychlorinated biphenyl \\
\hline PE & Plant Engineering \\
\hline QA & quality assurance \\
\hline$Q C$ & quality control \\
\hline $\mathrm{RCP}$ & reinforced concrete pipe \\
\hline RDL & reporting detection limit \\
\hline REAL & LLNL Radiological Environmental Analytical Laboratory \\
\hline
\end{tabular}




\section{Acronyms (Continued)}

$\begin{array}{ll}\text { S } & \text { solid } \\ \text { Sb } & \text { antimony } \\ \text { SDS } & \text { storm drain system } \\ \text { Se } & \text { selenium } \\ \text { SE } & \text { sediment } \\ \text { SO } & \text { soil } \\ \text { STLC } & \text { soluble threshold limit concentration } \\ \text { TCL } & \text { target compound list } \\ \text { Tl } & \text { thallium } \\ \text { TTLC } & \text { total threshold limit concentration } \\ \text { V } & \text { vanadium } \\ \text { VCP } & \text { vitrified clay pipe } \\ \text { Y } & \text { yes } \\ \text { Zn } & \text { zinc }\end{array}$




\section{LLNL Storm Drain Sampling Plan and \\ Follow-Up Cleanout and \\ Maintenance Proposal}

\section{Background}

In 1989, an LLNL Ground Water Project Baseline Public Health Assessment (BPHA) was conducted. The BPHA included the collection of an initial series of samples from selected storm drain catch basin sediments, storm drain surface water, and storm drain surface sediments. The BPHA Storm Drain Sampling Plan report listed the sampling protocol, sampling methods, analyses performed, and the sample results. An LLNL site map showing the sample locations was also included. Several samples with elevated contaminant levels are noted in the March 16, 1989 "Storm Drain Remediation Issues" memorandum by F. Hoffman. Recommendations resulting from this initial Storm Drain sampling effort and BPHA were to: (1) develop an ongoing program to monitor the LLNL storm drain system for potential contaminants; (2) develop and implement remediation of contaminated storm drain sediment; and (3) develop and implement a storm drain cleanout program.

The LLNL Storm Drain Sampling Plan and Follow-up Cleanout and Maintenance Proposal is being carried out as a best management practice and includes the following activities: (1) sample the Lawrence Livermore National Laboratory (LLNL) storm drain system (SDS) at the Livermore Site; (2) remediate all local sites found to be contaminated; (3) develop and implement an ongoing storm drain cleanout program; (4) develop an ongoing program to monitor the LLNL storm drain system for potential contaminants; and (5) document the LLNL storm drain system and assign responsibility for documenting changes and additions to the storm drain system. Items 2 through 5 are recommended follow-up activities to the sampling of the SDS. Activities 1 through 5 are an expanded summation of the recommended follow-up activities resulting from the initial sampling performed as part of the 1989 LLNL Ground Water Project BPHA.

\section{LLNL Storm Drain Grading and Drainage}

At the LLNL Livermore site, the natural slope of $1.5 \%$ grade toward the northwest is the controlling feature for all site grading and drainage. Since 1940, the natural drainage has been redirected and controlled as necessary in order to accommodate existing and new facilities.

The surface area of the Laboratory site is presently drained with a combination of subsurface drainage pipe networks, open surface channels, and swales (see LLNL Storm Drain System Site Map, LLNL drawing PLC78-099-052E, in Appendix A).

Portions of the southwest quadrant of the site are drained by a network of underground storm drain pipes and open surface channels that discharge through one of the two site outfalls into the Arroyo Seco drainage channel at the southwest corner of the site. The storm drains that discharge into the Arroyo Seco channel are sized to accommodate drainage generated by a "10-year-storm," which is standard municipal engineering practice. The Arroyo Seco channel has adequate flow capacity to accept anticipated flows generated by a "100-year-storm," and does not constitute a potential flood hazard to the LLNL Livermore site.

The other areas of the site (northwest, northeast, and southeast quadrants and a portion of the southwest quadrant) are drained by a network of underground storm drain pipes and open surface channels that drain into secondary channels that, in turn, drain into the relocated Arroyo Los Positas 
drainage channel that runs along the northern border of the site. The relocated Arroyo Los Positas drainage channel discharges into the Arroyo Los Positas drainage channel at the site's northwest outfall. The secondary channels generally have adequate flow capacity to accept anticipated flows generated by a "25-year-storm" Open channel improvements along the LINL reach of the relocated Arroyo Los Positas channel have upgraded the flow capacity to accommodate drainage generated by a "100-year-storm"; but various storm drain structures along the channel have not yet been upgraded.

A shallow surface swale along the southern border of the site (north side of East Avenue) drains surface storm runoff westward to the Arroyo Seco drainage channel. An open surface channel along the eastern border of the site (west side of Greenville road) drains surface storm runoff northward to the relocated Arroyo Los Positas drainage channel. The relocated Arroyo Los Positas drainage channel then runs westward along the northern border of the site to the outfall at the Northwest comer of the site.

\section{Storm Drain System at LLNL}

Underground pipe in the LLNL storm drain system consists of corrugated metal pipe (CMP), corrugated metal pipe-arched (CMPA), vitrified clay pipe (VCP), reinforced concrete pipe (RCP), some cast iron pipe (CIP), and some asbestos-impregnated cement pipe (ACP). Pipe diameters range from 6 inches to about 5 feet. Catch basins and manholes are located along each run of underground pipe in the network and act as access points to the system. They are generally installed where (1) two or more lines connect, (2) a line diameter changes, or (3) the grade or direction of flow changes. Since about 1980 , installed catch basins have been of the self-flushing type that do not have sumps. These precast, reinforced concrete catch basins are nominally 16 cubic feet (approximately 120 gallons). Older (pre1980) catch basins may have sumps that are approximately 6 -inches deep. Manholes are larger access points and are also constructed of precast reinforced concrete. There are approximately $740^{1}$ catch basins and approximately $94^{1}$ manholes that connect the underground pipe and open channels in the LLNL storm drain system. In areas of the site where there are no underground lines, open shallow surface swales and drainage channels direct the flow. LLNL drawing PLC78-099-052E, LLNL Storm Drain System Site Map, shows the existing underground storm drain lines, drainage channels, catch basins, and storm drain manholes. ${ }^{1}$

\section{LLNL Storm Drain Sampling Plan}

\section{Purpose for Sampling}

The purpose for sampling the LLNL storm drain system is to determine if any elevated contaminant levels of regulated hazardous and/or radioactive constituents may be present. The LLNL storm drain system will be sampled and characterized as a best management practice follow-up activity to the initial sampling that was conducted as part of the 1989 LLNL Ground Water Project BPHA.

Although waste is not intentionally discharged into the LLNL storm drain system, anecdotal information suggests that past discharges may have occurred. Some constituents may have become concentrated in catch basins or open channel sediment over time, even if they originally entered with runoff at nonhazardous concentrations, since the LLNL storm drain system is currently not cleaned out on a regular basis. Results from this sampling program and investigation will be useful in determining if there have been or continue to be any inappropriate discharges.

1 The catch basins, manholes, underground lines, drainage channels, and surface swales shown on LLNL drawing PLC78-099-052E, LLNL Storm Drain System Site Map, are current as of August 5, 1991. 
This sampling plan describes the procedures for adequate environmental sampling of the LLNL storm drain system. In the event that any constituents at hazardous concentrations are found, appropriate action will be taken which will include notification of regulators, clean-up and restoration, additional sampling, and cessation of any inappropriate ongoing discharges that might be found to exist.

\section{Sampling Strategy}

This sampling plan requires the possible collection and analyses of two different sample media type: (1) samples of the liquid component from some catch basins, if any standing liquid is present, and (2) samples of solids (including samples from catch basins, manholes, and open channel sites; and background samples from selected locations). Samples will be taken that adequately represent the spatial variations that may exist at each sample location.

\section{Initial Sampling}

This plan is a hierarchial sampling plan that consists of initially sampling selected sample locations on a one-time basis to preliminarily assess the entire LLNL storm drain system while limiting the number of sample locations. Starting at the highest elevation, the LINL storm drain system was reviewed and sample locations were selected at the down gradient junction of two intersecting lines or at the junction of a line and a network of lines. In addition, some sample locations were selected on the basis of past practices or known releases near a selected location. Each collected sample is considered to represent the sediment at that location and, furthermore, may represent sediment that is up gradient. If the sample results show that hazardous waste and/or radioactive constituents at levels above background are present, then appropriate remediation will be conducted and follow-up sampling will be performed. If any elevated contaminant levels of regulated hazardous and/or radioactive constituents are found, then on a case-by-case basis, additional sampling may be done to further characterize that portion of the SDS. If the sample results show that regulated hazardous and/or radioactive constituents either are nonexistent or are at background levels, then no further sampling will be done unless other information indicates that additional sampling may be required.

Storm drain influent flow varies temporally with respect to volume and content. Sediment in catch basins, manholes, and open channel sites that was deposited over time may have elevated concentrations resulting from accumulation. If open channel site sediment depth is relatively deep, it may be necessary to take sequential samples at various depths. If the sediment depth is shallow due to sediment being washed down gradient, then it will be necessary to take only one single or composite sample. Since catch basin and manhole sediment may have been laid down in uneven patterns that are unlikely to be uniformly mixed, constituent concentrations may vary over the accumulation area. Therefore, a systematic random composite sample may be required. This type of sampling involves dividing the accumulation area into sections, and then randomly sampling within each section. This sampling method provides more uniform coverage of the area than does random sampling, and further ensures that the accumulation area is more fully characterized.

\section{Additional Sampling}

If the results from sampling a particular sample location in the Initial Sampling indicate elevated contaminate levels, then on a case-by-case basis additional upgradient sample locations will be selected for sampling in subsequent sampling events.

\section{QA Sampling}

Off-site background samples will be taken to establish a basis for comparison between the collected field samples and the surrounding area. The Quality Assurance/Quality Control (QA/QC) protocol described in the "Sampling Procedure" outlines the necessary QA sampling to ensure the integrity of the sampling plan and procedures. 


\section{Applicable Regulations and Methods}

The following is a list of regulations and methods that are applicable to sampling activities.

CCR Title 22

Division 4, Environmental Heaith

40 CFR Part 136

Guidelines for Establishing Test Procedures for the Analysis of Pollutants

40 CFR Part 261

Identification and Listing of Hazardous Waste

EPA-SW-846

Test Methods for Evaluating Solid Waste

EPA-600/4-79-020

Methods for Chemical Analysis of Water and Wastes

EPA-600/4-82-029

Handbook for Sampling and Sample Preservation of Water and Wastewater

EPA-600/4-82-057 Methods for Organic Chemical Analysis of Municipal and Industrial Wastewater

\section{Media to be Sampled and Sample Types}

All field samples (FS) will consist of either solid (S) or liquid (L) samples. Solid samples will be either sediment (SE) or soil (SO) media. Liquid samples that result from storm runoff or area runoff due to LLNL standard operating procedures (i.e., irrigation, steam cleaning, etc.) will contain varying percentages of solids. ${ }^{2}$ Collected Quality Assurance (QA) samples, including duplicate samples (DP) and field blank (FB) samples, may be either solid or liquid samples. QA background field samples (BF) will be taken to establish a basis for comparison between the collected field samples and the surrounding area.

\section{Target Constituents}

The target constituents for solid samples and liquid samples are listed in Table 1.

$\overline{2}$ The analytical method for liquid samples that contain varying percentages of solids will be determined by the analytical laboratory after the percent of solids has been determined.

Table 1. Target constituents for solid and liquidª samples.

Metals $^{\mathrm{b}}$ (TTLC and STLC)
Volatile TCL organics
Semi-volatile TCL organics
Pesticides and PCBsc
Radionuclides
Gross alpha
Gross beta
Gamma
Tritium

a The determination of whether a sample that contains varying percentages of solids is a liquid or solid sample will be determined by the analytical laboratory after the percent of solids has been determined.

b California Assessment Manual (CAM) Metals - (17 metals): Sb, As, Ba, Be, Cd, Cr, Co, Cu, Pb, Hg, Mo, Ni, Se, $\mathrm{Ag}, \mathrm{Tl}, \mathrm{V}, \mathrm{Zn}$

c Organochlorine pesticides and polychlorinated biphenyls. 


\section{Area to be Sampled}

The area to be sampled consists of the entire LLNL storm drain system that is located within the LLNL Livermore site. Refer to LLNL drawing PLC78-099-052E, LLNL Storm Drain System Site Map in Appendix A, which shows the LLNL Site and the LLNL storm drain system. Field samples will be taken from selected catch basins, manholes, open channel sites, and other locations that are within the LLNL storm drain system. Quality assurance samples will be taken from areas within and outside the LINL Livermore site.

\section{Sampling Procedure}

\section{Quality Assurance/Quality Control}

Quality Assurance (QA) and Quality Control $(Q C)$ shall be integrated throughout the Storm Drain Sampling Program. LLNL policies and procedures, including those outlined in the Health and Safety Manual, shall be followed.

Duplicate Samples. Duplicate samples provide information on the precision of the sampling, sample preservation, shipping, and analytical techniques and procedures. The total number of duplicate samples shall be equal to or greater than 10 percent of the total number of samples. A minimum of one duplicate sample shall be included for every 10 samples collected (one duplicate sample shall be taken even when fewer than 10 samples are collected).

Blank Samples. A minimum of one liquid field blank sample shall be used for every daily set of liquid samples collected. Liquid field blanks shall be carbon-filtered distilled water.

Background Samples. Background field samples shall be taken to establish a basis for comparison between the collected field samples and the surrounding area. Background field samples shall be solid samples of undisturbed native soil taken from selected locations. Background samples shall be taken from areas outside the SDS and/or from off-site locations.

Documentation and Reporting. If the sample does not require expedited analyses, then written and signed analytical reports, with accompanying chain-of-custody documentation and QA/QC data, shall be received by the designated LLNL representative within 20 calendar days of sample submittal. If the sample does require expedited analyses, then preliminary sample results of analyses are to be reported verbally, by FAX, and/or by electronic data transfer to the designated LLNL representative immediately upon completion of the analyses. The preliminary results are to be followed within 5 working days by a written and signed analytical report that contains complete QA/QC data and chain-of-custody documentation.

Written analytical reports, at a minimum, are to include the following:

Sample data

- LINL sample ID number or unique sample identifier

- sample collection date

- date the laboratory received the sample

- sample analysis date

- requested analyses method number(s)

- date of sample digestion

- sample dilution values

- laboratory's sample log number

- sample description

- chain-of-custody form number

- analytical results 
- QA/QC data

- signature of lab director or responsible lab representative

$Q A / O C$ data (for each analyte)

- Laboratory control standards (LCS) data: batch number, method number, date of analysis, instrument ID number, analyst's ID number, and percent recovery

- Matrix QC precision (lab split/duplicate sample) data: batch number, method number, date of analysis, instrument ID number, analyst's ID number, and percent relative difference

- Matrix QC accuracy (spike) data: batch number, method number, date of analysis, instrument ID number, analyst's ID number, and percent recovery

- Method blank data: batch number, method number, date of analysis, instrument ID number, analyst's ID number, and method blank results

- Reporting detection limit (RD́L)

- Minimum detection limit (MDL)

The analytical laboratory must provide the designated LLNL representative with the control limits or acceptance criteria used for LCS, matrix QC precision and accuracy, method blanks, and reporting detection limit data used to determine acceptance. If the control limits are unacceptable to the LLNL representative, the analytical laboratory shall use contract RDL limits based on EPA or Contract Laboratory Program recommendations. All analyses shall be reported in units specified by the designated LLNL representative. Chain-of-custody forms shall be required for all samples collected for analyses under the Storm Drain Sampling Program. Each set of sample results received from an analytical laboratory shall be accompanied by the applicable QC data.

QA/QC Policy and Procedures. Policies and procedures that shall be used for QC inciude the following:

- Personnel shall be trained and knowledgeable of appropriate LLNL. Health and Safety issues.

- Personnel shall be trained and shall follow standard operating procedures for the collection, handling, and preservation of environmental samples that may exhibit hazardous waste characteristics.

- Only certified analytical laboratories shall be used to conduct nonradionuclide analyses of samples.

- All activities shall be done in accordance with EPA regulations for sampling and analysis.

- All work activities shall be done in accordance with LLNL Health and Safety Manual requirements.

- All work activities shall be done in accordance with LLNL policies and procedures.

- All required documentation, including chain-of-custody forms, shall be created and organized for ease of review.

\section{Sampling Method}

Solid samples from storm drain catch basins and manholes will consist of two samples that are collected in squat widemouth glass jars and one sample that will be collected in a stainless steel tube. The sample in the stainless steel tube will be analyzed for volatile TCL organics and semi-volatile TCL organics. The two samples in the glass jars will be analyzed for the other target constituents listed in Table 1. These samples will be collected with stainless steel tools or disposable plastic scoops. Due to the variety of materials that potentially will be sampled by this method, the selection of sampling equipment will occur on a sample-specific basis. Liquid that may be standing in a storm drain catch basin or manhole will be collected in glass jars.

Solid samples from open channel sites will consist of two samples that are collected in squat widemouth glass jars and one core sample that will be collected with a stainless steel core sampler from a hand-excavated boring. Borings may extend from the surface to a depth of a few feet. Core samples 
will be collected with a hammer-driven, stainless steel core barrel. The core barrel will be fitted with a stainless steel tube that will retain the sample. Analyses will be the same as for those collected from catch basins and manholes.

After collection of the samples in stainless steel tubes, the ends of each tube will be covered with clean Teflon sheeting and capped with plastic end caps. Each plastic end cap will be sealed with duct tape that will extend to the sample label placed on the side of the tube.

Sample collection equipment shall be thoroughly cleaned and decontaminated prior to taking a sample and between each sampling event. Sufficient sampling equipment will be provided to minimize field cleaning of equipment. All stainless steel sleeves will be cleaned and protected prior to being transported to the field. All other sample containers will be pre-cleaned or supplied by the analytical laboratory; therefore, no field cleaning of any sample containers will be necessary.

All ice chests shall be washed with laboratory detergent (interior and exterior), rinsed with tap water, and air dried before storage or use.

The method(s) for sample collection, equipment cleaning, sample container cleaning, sampling equipment use and operation, vehicle use and cleaning, and other sampling method considerations shall be in accordance with the EPD Field Sampling Training Manual, 92044.01.

\section{Sample Labeling}

Each sample that is collected shall be labeled with at least the following information:

- unique sample ID number

- sample collection date

- time sample was taken

- sample location

- requested analyses

- identity of the sampler(s)

\section{Sample Transportation, Treatment, and Storage}

All environmental samples and QA samples will be protected by appropriate protective coverings and isolated from external contaminants. Samples will be stored in ice chests, with ice when appropriate, and transported to an analytical laboratory as soon as practical and within the minimum hold time for the specified analysis. A courier service will be used to transport samples between LLNL and the analytical laboratory.

\section{Chain-of-Custody Forms and Field Tracking Forms}

The sampler(s) shall fill out a chain-of-custody form at the time the sample is collected. The chain-of-custody form shall remain with the sample at all times. The chain-of-custody form shall be signed and dated each time a sample is transferred from one individual to another.

\section{Sampling Locations}

The individual sampling locations may consist of catch basins, manholes, open channel sites, selected background locations, and other locations. Sampling locations are listed in Table 2 and also shown on the Sampling Location Site Map (Appendix B). A copy of the LINL Storm Drain System Site Map (LLNL drawing PLC78-099-052E) has been marked up to indicate the locations of all the sample locations. Triangles indicate field sample locations and asterisks indicate background field sample locations. LLNL drawing PLC78-099-052E shows all documented manholes, catch basins, and open channel swales. The manholes are indicated by solid circles, the catch basins are indicated by open circles, and the open channel swales are indicated by solid lines with flow indication arrows. This drawing has a grid that divides the LLNL site into grid blocks. The vertical axis is divided from 10 through 90 and the horizontal axis is divided from 0 through 600 . Each block has a unique grid block 
Table 2. Sampling Locations in the LLNL Storm Drain System.

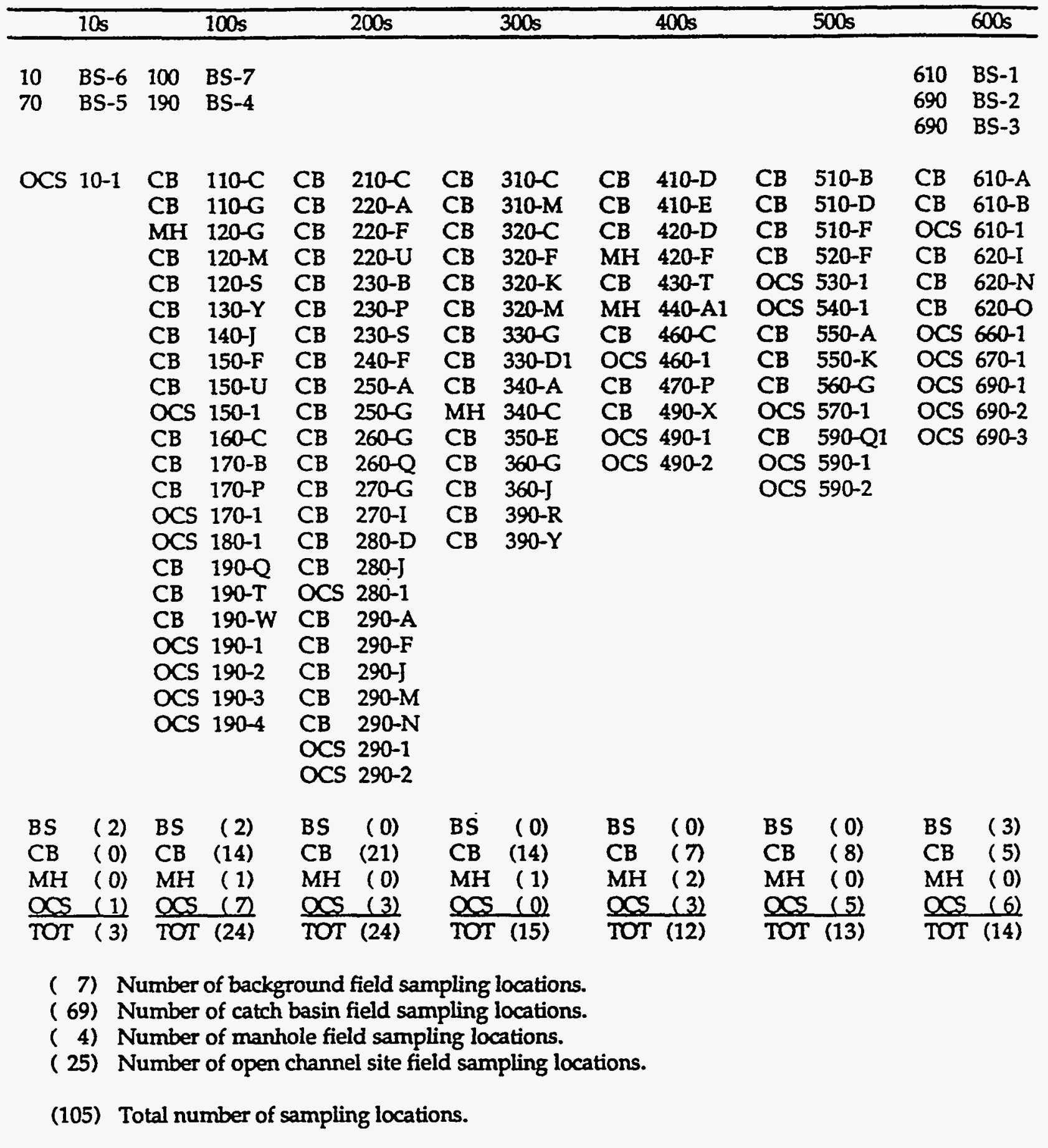

number. Manholes and catch basins, within each grid block, are uniquely labeled A, B, C, etc. A combination of the grid block number and the letter designation provides a unique label for each manhole or catch basin, e.g., catch basin 190-W. Open Channel Site sample locations, within each grid block, have been sequentially numbered 1,2,3, etc. A combination of the grid block number and the sequence number provides a unique label for each Open Channel Site sample location, e.g., $190-1$. Background field sample locations are sequentially numbered and have the prefix BS, e.g., BS-1. 
The Location Number, Location Type, Location Type ID, and Sample Purpose for each sampling location will be entered on a Sampling Location Data Sheet (Appendix C) prior to sampling. The Sample Media, Bottom information, Sampler, Time, Date, and Sample ID will be entered at the time the sample is taken. An example of a completed Sampling Location Data Sheet is shown in Table 3.

Table 3. Sampling location data sheet for the LLNL storm drain system.

\section{Sampling Location Data Sheet}

\begin{tabular}{|c|c|c|c|c|c|c|c|c|c|}
\hline $\begin{array}{l}\text { Location } \\
\text { Number }^{2}\end{array}$ & $\begin{array}{l}\text { Location } \\
\text { Type }\end{array}$ & $\begin{array}{l}\text { Location } \\
\text { Type } \\
\text { IDc }\end{array}$ & $\begin{array}{l}\text { Sample } \\
\text { Purposed }\end{array}$ & $\begin{array}{l}\text { Sample } \\
\text { Media }\end{array}$ & Bottom & Samplerg & Timeh & Date $^{i}$ & $\begin{array}{c}\text { Sample } \\
\text { IDj }\end{array}$ \\
\hline 001 & $\mathrm{CB}$ & $510-\mathrm{D}$ & FS & L S & YNNA & PK/BI & $8: 30$ & $08 / 15 / 91$ & \\
\hline 002 & $\mathrm{MH}$ & $520-\mathrm{F}$ & FS & L S & $\underline{Y N N A}$ & $\mathrm{PK} / \mathrm{BI}$ & $9: 15$ & $\underline{08 / 15 / 91}$ & \\
\hline 003 & OCS & $150-1$ & FS & L S & YN NA & PK & 10.00 & $\underline{08 / 15 / 91}$ & \\
\hline 004 & $\mathrm{O}$ & $0-1$ & FS & L S & YNNA & $\mathrm{PK} / \mathrm{BI}$ & $10: 45$ & $\underline{08 / 15 / 91}$ & \\
\hline 005 & BS & BS-1 & FS & L S & $\underline{Y N}$ NA & PK & $11: 30$ & $\underline{08 / 15 / 91}$ & \\
\hline 006 & $\mathrm{CB}$ & $420-D$ & FS & L S & YNNA & PK/BI & $13: 30$ & $\underline{08 / 15 / 91}$ & \\
\hline 006 & $C B$ & $420-D$ & DP & L S & $\underline{Y N N A}$ & $\mathrm{PK} / \mathrm{B}[$ & $13: 40$ & $08 / 15 / 91$ & \\
\hline $\mathrm{QA}$ & $\mathrm{QA}$ & $=$ & $\mathrm{FB}$ & $\mathrm{LS}$ & YNNA & $\mathrm{PK} / \mathrm{BI}$ & 14:15 & $\underline{08 / 15 / 91}$ & \\
\hline
\end{tabular}

a Enter the Location Number. Also note Location Number on the Sampling Location Site Map. Enter QA for field blanks.

b Enter the appropriate Location Type: $B S=$ Background Sample; $C B=$ Catch Basin; $M H=$ Manhole; $O=$ Other; OCS = Open Channel Site; $Q A=$ Quality Assurance.

c Enter the appropriate Location Type ID: CB and MH Location Type IDs refer to catch basins or manholes that are shown on LLNL drawing PLC78-099-052E, LLNL Storm Drain System Site Map. OCS Location Type IDs refer to the grid block location and a sequence \# shown on the Sampling Location Site Map (Appendix B). BS and $O$ Location Type IDs-refer to background sample sites or other locations shown on the Sampling Location Site Map (Appendix B).

d Enter the appropriate Sample Purpose: BF = Background Field; DP = Duplicate; FS = Field Sample; $\mathrm{FB}=$ Field Blank.

e Circle appropriate Sample Media that was taken: $\mathrm{L}=$ Liquid; $\mathrm{S}=$ Solid.

f Circle $Y($ Yes) or $\mathrm{N}(\mathrm{No})$ to indicate if there is a bottom in the sampled catch basin. Circle NA (Not Applicable) if the Sample Location is not a catch basin or manhole.

8 Enter the initials of the person or persons who took the sample, e.g., PK/BJ.

$h$ Enter the time that the sample was taken. Use 24-hour notation.

i Enter the date that the sample was taken. Use MM/DD/YY notation.

j Enter the unique Sample ID. 


\section{Analytical Laboratories}

The following list of analytical laboratories will be considered to process and analyze the collected samples. Only laboratories that are State certified for specified nonradionuclide analytical method will be used.

Laboratory

Brown and Caldwell Analytical Laboratory (BCA)

Clayton Environmental Cō̄sultants (CEC)

Analytical Laboratory

LLNL Environmental-Analytical Sciences Laboratory (EAS)

\section{Contact Person(s)}

Larry Penfold

(415) $428-2300$

Maryann Gambino

(415) $426-2600$

Price Russ

(415) $422-6671$

Pager \# 6197

Dave Silberman

(415) 422-2100

Pager * 5661

Susan Gagner

(415) 423-6008

Rod Dougan

(415) $422-6648$

Building Page 2-8151

Laboratory (REAL)

Dinkar Kharkar

TMA Norcal Analytical Laboratory (TMA/Norcal)

(415) $235-2633$

\section{Sampling Materials, Equipment, and Supplies}

Table 4 provides the descriptions of necessary sampling materials, equipment, and supplies, including the stock numbers for ordering. Equivalent items may be substituted as necessary.

Table 4. Sampling materials, equipment, and supplies.

\begin{tabular}{|c|c|c|}
\hline $\begin{array}{l}\text { General } \\
\text { Quantity }\end{array}$ & Item & $\begin{array}{l}\text { Stock } \\
\text { Number }\end{array}$ \\
\hline 4 pair & $\begin{array}{l}\text { Leather gloves, } \mathrm{M} \\
\text { Leather gloves, } \mathrm{L} \\
\text { Leather gloves, } \mathrm{XL}\end{array}$ & $\begin{array}{l}8415-49220 \\
8415-49219 \\
8415-50328\end{array}$ \\
\hline 4 each & Hand trowel, ss & Fabricated \\
\hline 1 roll & Teflon tape - $3 \mathrm{mil} \times 4^{\prime \prime} \times 60^{\prime}$ & Order \\
\hline 8 rolls & Vinyl tape, red $-1 / 2^{n}$ wide & 5970-56200 \\
\hline 4 rolls & Duct tape, black - $1^{n}$ wide & $7510-53251$ \\
\hline 4 rolls & Acetate tape, see through $-2^{n}$ wide & $7510-12334$ \\
\hline 4 each & Marker pen, black - Sharpie & $7520-62156$ \\
\hline 3 each & Filter cartridge, rinsewater & $4180-63126$ \\
\hline 2 boxes & Detergent, Tide & $7930-30299$ \\
\hline as req & Wipes-Kay Dry & $7999-63447$ \\
\hline 2 each & Ice chest-various sizes & Order \\
\hline as req & Ice, crushed & NA \\
\hline as req & Preservative & NA \\
\hline as req & Chain-of-custody form & NA \\
\hline as req & Radiation analyses form & $7600-69183$ \\
\hline as req & EAS waste analysis request form & $L L-6267$ \\
\hline as req & Field tracking form & NA \\
\hline
\end{tabular}




\begin{tabular}{lll}
$\begin{array}{l}\text { Per Sample } \\
\text { Location } \\
\text { Ouantity }\end{array}$ & Item & Stock \\
& & Number \\
2 pair & Vinyl gloves (disposable), S & $8415-64993$ \\
& Vinyl gloves (disposable), M & $8415-64994$ \\
& Vinyl gloves (disposable), L & $8415-64995$ \\
1 each & Tube, 0.049"tk ss $-15^{n}$ OD, $\times 6^{\prime \prime} 1$ & Order \\
2 each & Tube caps, polyethylene $-1.5^{n}$ OD & Order \\
2 each & Glass jar, squat widemouth -4 oz & $\mathbf{8 1 2 5 - 2 7 7 9 9}$ \\
3 each & Labels, self adhesive $-1.5^{n} \times 2.75^{n}$ & $7530-53182$ \\
3 each & Plastic bag, sealable $-6^{n} \times 8^{n}$ & $\mathbf{8 1 0 5 - 6 0 3 9 8}$ \\
1 each & Plastic bag, nonsealable $-6^{n} \times 12^{n}$ & $8105-27699$ \\
\hline
\end{tabular}

\section{Follow-Up Activities}

The following are recommended follow-up activities to sampling the LLNL storm drain system.

\section{Remediation of LLNL Storm Drain System}

If necessary, appropriate remediation and cleanup of all contaminated locations will be performed by the Environmental Restoration Division.

\section{LLNL Storm Drain Cleanout and Disposal Recommendations}

The Maintenance Operations Division in the Maintenance/Operations Department of Plant Engineering has the responsibility for cleanout of the LLNL storm drain system and disposal of nonhazardous debris. Cleanout has been performed on an "as needed" basis, and no set frequency has been established. It is recommended that the Maintenance/Operations Department review the results of the LLNL Storm Drain Sampling Program, inspect the SDS, and then decide if a set schedule for cleanout is required or if the current "as needed" basis is appropriate. The Maintenance/Operations Department will continue to have the primary responsibility for cleanout of the LLNL storm drain system, and LLNL emergency response personnel would respond if a spill of hazardous material to the SDS were to occur.

\section{Future and Ongoing Monitoring of the LLNL Storm Drain System}

Future and ongoing inspections, surveillance, and monitoring of the LLNL storm drain system will be conducted by the Environmental Monitoring Group of the Environmental Protection Department. A sampling plan that addresses the necessary sampling protocol, parameters, analytes, and frequency to adequately sample the LINL storm drain system on a regular and ongoing basis will be developed as part of the overall LLNL Storm Drain Monitoring.Program.

\section{Documentation of LLNL Storm Drain System}

The Utilities Division in the Maintenance/Operations Department of Plant Engineering has the responsibility for maintenance of the LLNL storm drain system and for documenting and updating the LLNL Storm Drain System Site Map, LLNL drawing PLC78-099-052E. 


\section{Appendix A}

LLNL Storm Drain System Site Map

(LLNL drawing PLC78-099-052E)

B-16 


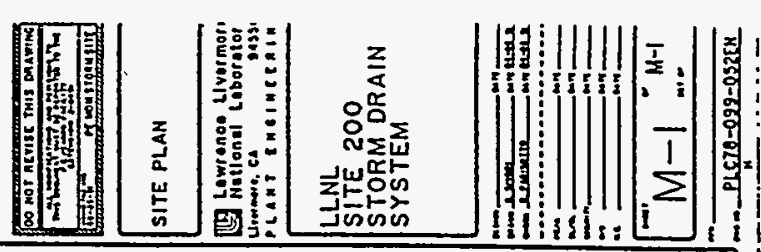

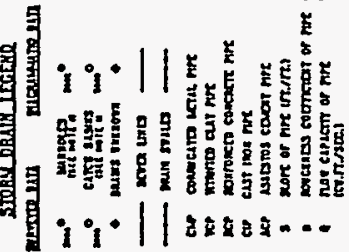
(I) $4=0$

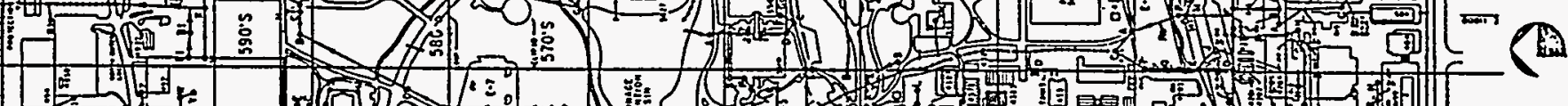

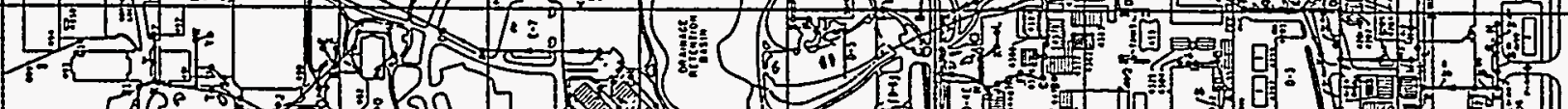
(1) 5 ( (1)

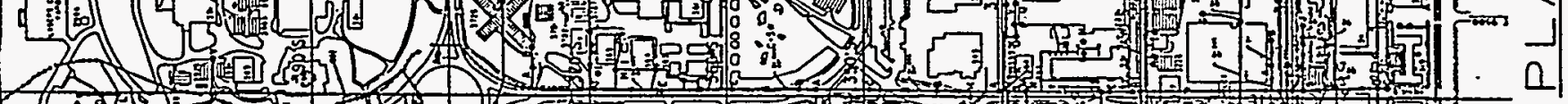

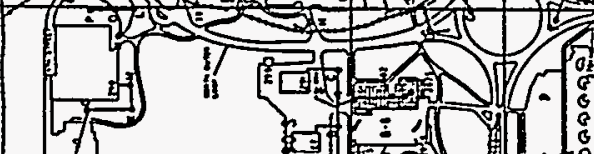

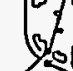

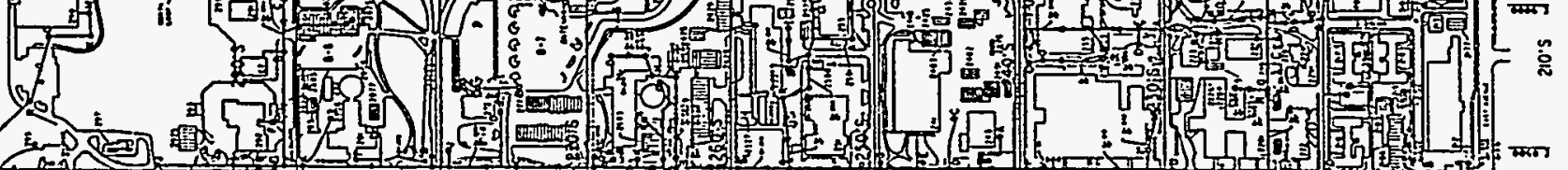

-

(1)

(12)

Jer

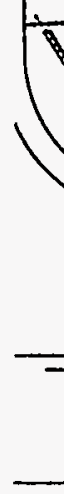
E
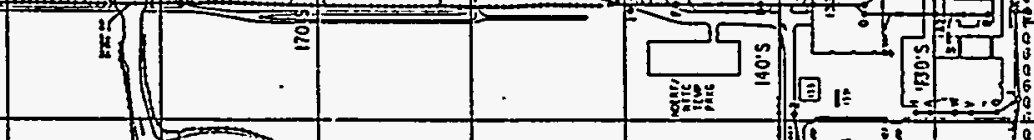


\section{Appendix B}

Sampling Location Site Map

B-1

B-18 


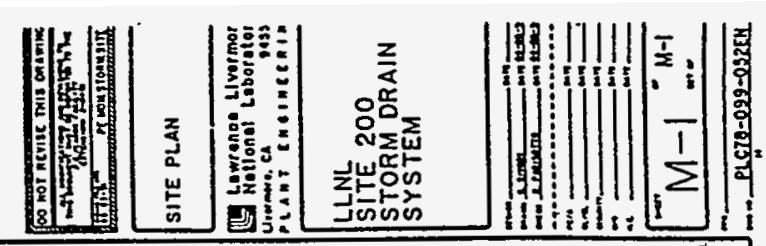

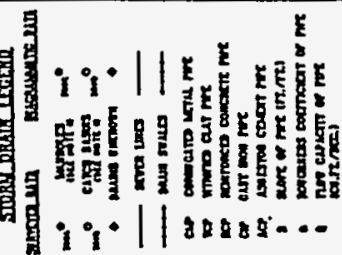
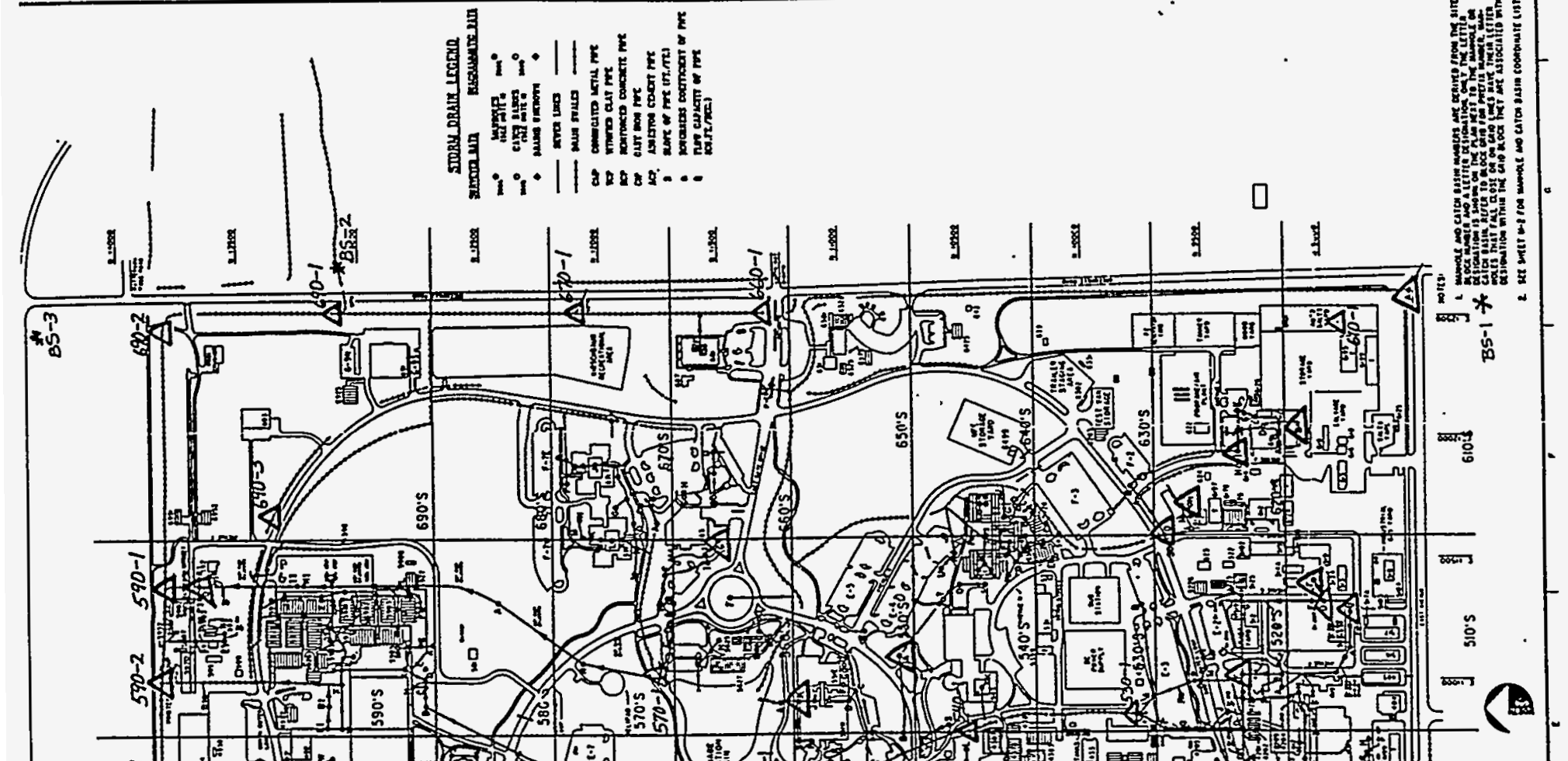

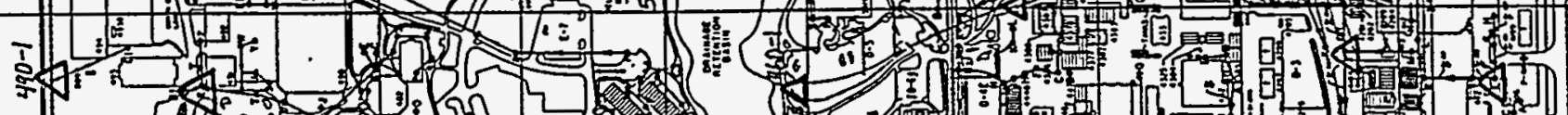

7.75

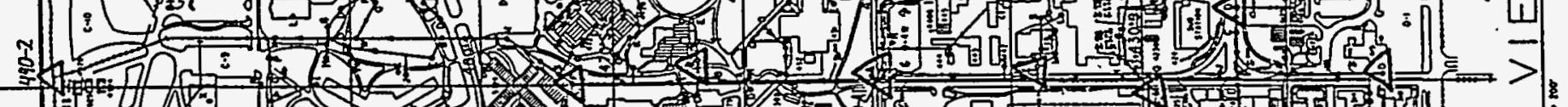
(1) $-44=1$ (1)

*1 (5) 9 (1) 


\section{Appendix C}

\section{Sampling Location Data Sheet}

C-1 


\section{Sampling Location Data Sheet}

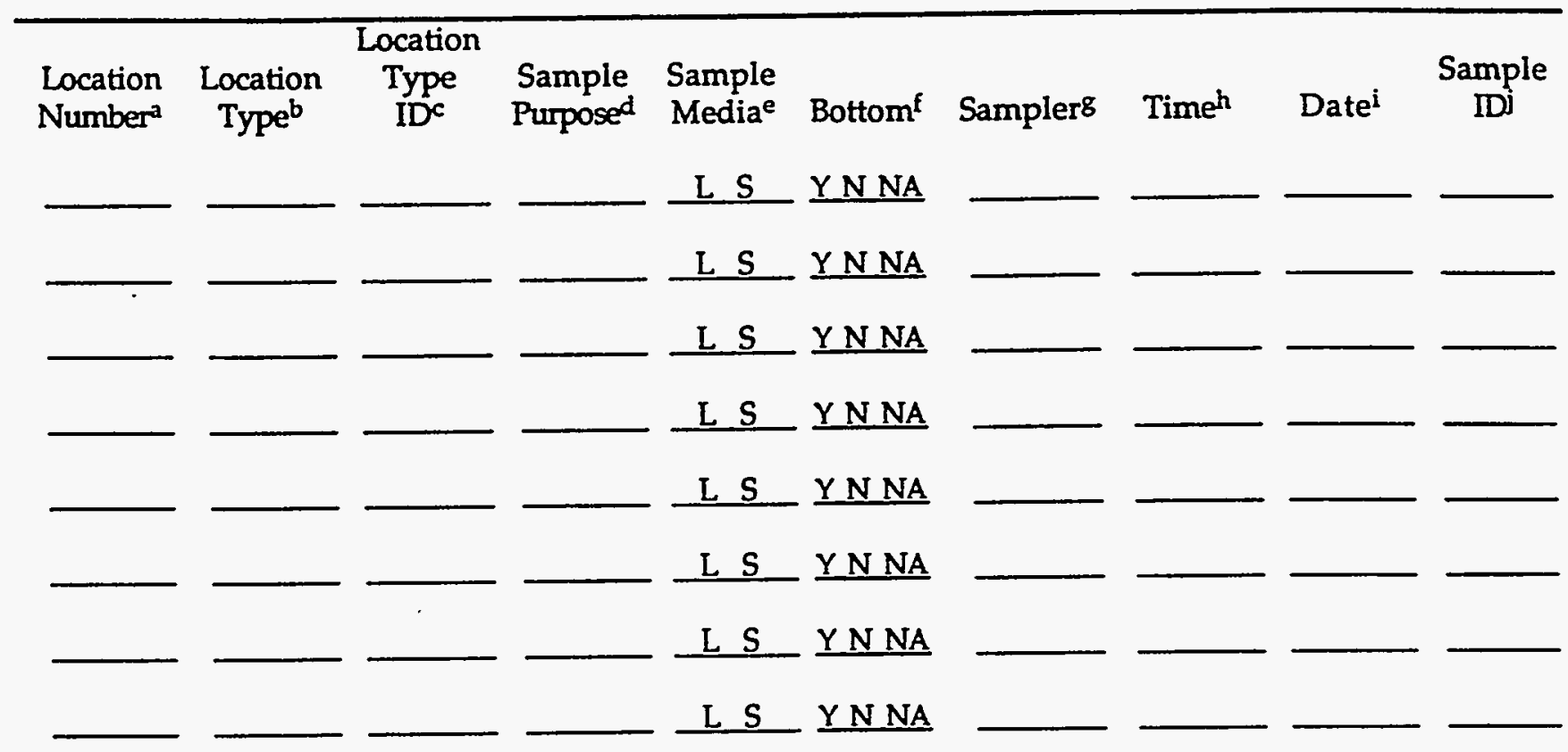

a Enter the Location Number. Also note Location Number on the Sampling Location Site Map. Enter QA for field blanks.

b Enter the appropriate Location Type: $B S=$ Background Sample; $C B=$ Catch Basin; $M H=$ Manhole; $O=$ Other; $O C S=$ Open Channel Site; $Q A=$ Quality Assurance.

c Enter the appropriate Location Type ID: CB and MH Location Type IDs refer to catch basins or manholes that are shown on LLNL drawing PLC78-099-052E, LLNL Storm Drain System Site Map. OCS Location Type IDs refer to the grid block location and a sequence \# shown on the Sampling Location Site Map (Appendix B). BS and $O$ Location Type IDs refer to background sample sites or other locations that are shown on the Sampling Location Site Map (Appendix B).

d Enter the appropriate Sample Purpose: BF =-Background Field; DP = Duplicate; FS = Field Sample; $\mathrm{FB}=$ Field Blank.

e Circle appropriate Sample Media that was taken: $L=$ Liquid; $S=$ Solid.

I Circle $Y$ (Yes) or $N$ (No) to indicate if there is a bottom in the sampled catch basin. Circle NA (Not Applicable) if the Sample Location is not a catch basin or manhole.

g Enter the initials of the person or persons who took the sample, e.g., PK/BJ.

h Enter the time that the sample was taken. Use 24-hour notation.

1 Enter the date that the sample was taken. Use MM/DD/YY notation.

i Enter the unique Sample ID. 


\section{Appendix C}

\section{Surveillance Soils and Sediment Sampling Procedure}




\begin{tabular}{|c|c|c|}
\hline $\begin{array}{c}\text { REVISION 3 } \\
\text { FINAL }\end{array}$ & $\begin{array}{c}\text { EMP-S-S } \\
\text { Soil \& Arroyo Sediment Sampling }\end{array}$ & $\begin{array}{c}\text { Effective date: 01/04/93 } \\
\text { Page } 1 \text { of } 16\end{array}$ \\
\hline
\end{tabular}

\section{EMP-S-S}

SOIL \& ARROYO SEDIMENT SAMPLING

Prepared by:

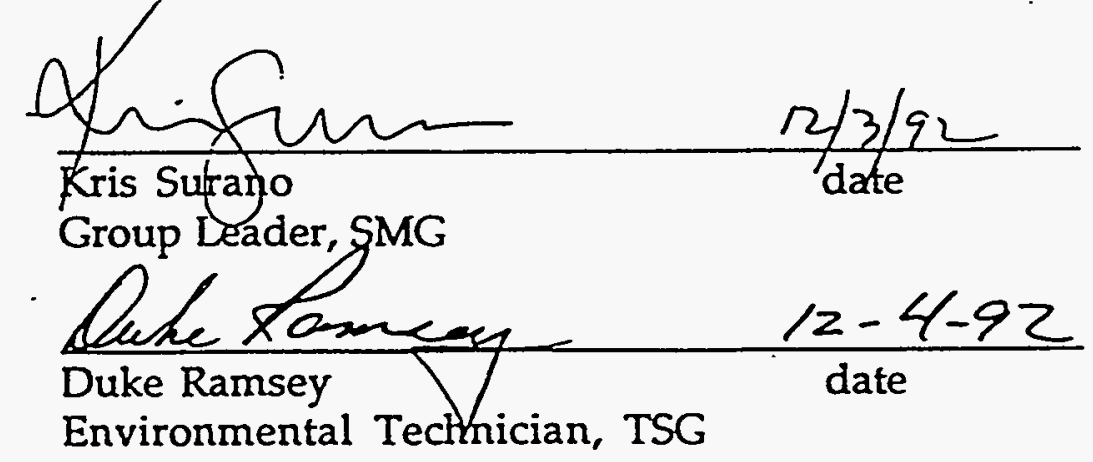

Reviewed by:

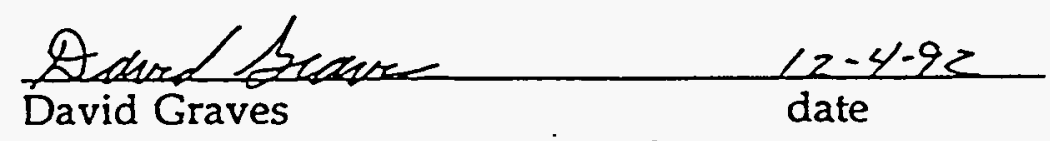

Environmental Technician; TSG

$\frac{\text { Pasiallhouse }}{\text { Paris Althouse }} \frac{12-4-92}{\text { date }}$

Environmental Technician, TSG

Approved by:

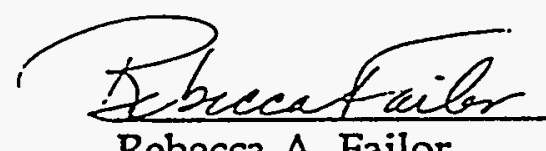

Rebecca A. Failor

Section Leader, EMS

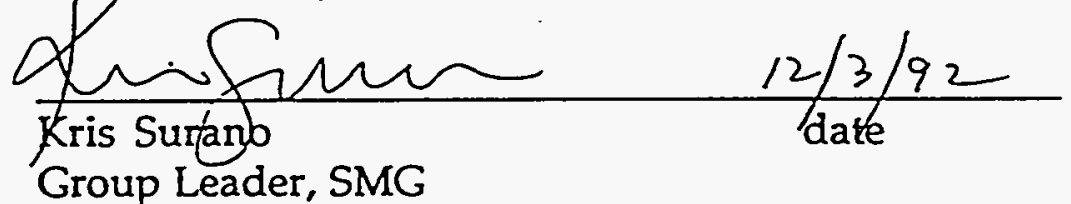

Group Leader, SMG

Lucencla M Gavera. 1218192
$\begin{aligned} & \text { Lucinda Garcia } \\ & \text { Quality Assurance Coordinator, EMS }\end{aligned}$




\begin{tabular}{|c|c|c|}
\hline $\begin{array}{c}\text { REVISION 3 } \\
\text { FINAL }\end{array}$ & $\begin{array}{c}\text { EMP-S-S } \\
\text { Soil \& Arroyo Sediment Sampling }\end{array}$ & $\begin{array}{c}\text { Effective date: 01/04/93 } \\
\text { Page 2 of 16 }\end{array}$ \\
\hline
\end{tabular}

\section{PURPOSE}

This procedure describes the methods to be used in collecting surface soil and arroyo sediment samples for EMS environmental surveillance activities at LLNL, in the Livermore Valley, and at Site 300 . This procedure applies to both routine and non-routine sampling unless the non-routine sampling plan specifically states otherwise.

\section{DISCUSSION}

Soil is an integrating medium that can contain pollutants originally released directly to the ground, to the air, or though liquid effluents. Reference 1, on which much of EMS monitoring is based, states that "Periodic sampling and analysis of indicator materials, such as soil ... should be performed to determine of there is measurable long-term buildup of radionuclides in the terrestrial environment." Since 1972, surface soil sampling in the vicinity of LLNL and Site 300 has been part of a continuing LINL monitoring program designed to measure any changes in environmental levels of radioactivity and to evaluate any increase in radioactivity that might have resulted from LLNL operations. Surface soil samples are also analyzed for beryllium, a potentially toxic metal used at LLNL and Site 300.

EMS soil sampling locations are chosen to coincide with air sampling locations or to give coverage to areas of special concern, including areas of known past contamination (Reference 2). The use of constant sampling locations allows better trending of data and closer monitoring of areas of concern. All soil sampling is conducted according to this procedure.

According to Reference 1, The sampling of sedimentary material from streams or ponds can provide an indication of the accumulation of undissolved radionuclides in the aquatic environment. The accumulation of radioactive materials in sediment can lead to exposure of humans through ingestion of aquatic species, through sediment resuspension into drinking water supplies, or as an external radiation source..." Sediments also have the potential to concentrate nonradioactive constituents present in storm water runoff. Sediment sampling, followed by radiological and chemical analyses, helps EMS determine if significant buildups of pollutants have occurred in nearby arroyos.

Annual sediment samples have been collected from selected arroyos and other drainages at and around LLNL since 1988. Sediments from 11 major LLNL drainages curreritly are sampled. The sediment sampling locations coincide with storm water runoff sampling locations so results from the sampling of these two related media can be compared (Reference 3). Analyses have been conducted on both radioactive and nonradioactive constituents of sediment samples every year since the inception of sediment monitoring. All sediment sampling is conducted according to this procedure.

\section{REFERENCES}

1. DOE/EH-0173T, Environmental Regulatory Guide for Radiological Effluent Monitoring and Enoironmental Suroeillance

2. EMP-S-L, Soil and Arroyo Sampling Locations

3. EMP-RO-L, Storm Water Runoff Sampling Locations

4. EMP-QA-CD, Design Change Packages

5. EMP-QA-NCR, Nonconformance Reports

6. EMP-QA-D, Data Analysis

7. EMP-QA-AER, Annual Environmental Report .

8. EMP-QA-SL, Sample Location Designators

9. EMP-QA-SA, Self Assessments

10. EMP-QA-SD, Sample Tracking Documentation

11. EMP-QA-DC, Document Retention Center 


\begin{tabular}{|c|c|c|}
\hline $\begin{array}{c}\text { REVISION 3 } \\
\text { FINAL }\end{array}$ & EMP-S-S & $\begin{array}{c}\text { Effective date: } 01 / 04 / 93 \\
\text { Page } 3 \text { of } 16\end{array}$ \\
\hline
\end{tabular}

12. EMP-HS-300, Site 300 Health and Safety Work Procedure

13. EMP-QA-S, Quality Assurance Samples

14. EMP-QA-DM, Data Management

15. EMP-QA-CS, Courier Seroice Protocol

16. Sims, et al., Enoironmental Report for 1990 (UCRL-50027-90)

\section{DEFINITIONS}

Arroyo: A deep gully cut by an intermittent stream.

Auger. Tool used to bore holes in the ground. Soil/sediment samples can be collected from the auger bit after it is withdrawn from the ground.

Core Sampler: An impact-driven soil sampling device for collecting an intact, undisturbed cylinder of surface soil from the ground.

Sample Identifier: The four-field code that identifies an individual sample to an analytical laboratory. It is made up of the sample location designator (two fields), a sample number, and a media code (L-ZON7-01-SO, for example).

Sediment: Finely divided solid material that settles to the bottom of a liquid.

Sediment sample: A aliquot of arroyo sediment taken with a split-spoon sampler driven into the sediment to a depth of $2 \mathrm{ft}$.

Slide Hammer: A device used to pound soil/sediment sampling equipment into the ground. It is made of a heavy weight which slides up and down on a smooth rod, striking the soil sampling equipment with a descending blow. Repetitive blows can drive sampling equipment to the necessary depths even when the ground has hardened.

Soil: The top layer of earth, suitable for the growth of plants.

Soil sample. An aliquot of surface soil collected to a depth of $5 \mathrm{~cm}$ because surface deposition from the air is the primary pathway for potential soil contamination.

Split-Spoon Sampler: A specialized soil sampling device which can be pounded into the ground and extracted with the soil core intact. The sampler can be opened longitudinally to reveal the intact soil core, which can then be collected untouched for analysis.

\section{RESPONSIBILITIES}

\subsection{EMS ENVIRONMENTAL ANALYST (SOILS)}

The Environmental Analyst assigned responsibility for the soil and sediment monitoring networks will decide when and where samples are to be collected. Sampling locations are described in Reference 2 . The Analyst must periodically review the network sampling locations, the sample collection schedule, soil and sediment collection methods, quality assurance (QA) sampling, and sample analytes for technical suitability in the context of EMS goals and missions. Furthermore, the Analyst is responsible for assessing the need to sample newly discovered areas of known or suspected contamination. The Analyst is responsible for submitting design changes regarding the network sampling locations, schedules, and sampling methods (Reference 


\begin{tabular}{|c|c||c|}
\hline $\begin{array}{c}\text { REVISION 3 } \\
\text { FINAL }\end{array}$ & EMP-S-S & $\begin{array}{c}\text { Effective date: 01/04/93 } \\
\text { Page 4 of 16 }\end{array}$ \\
\hline
\end{tabular}

4). The Analyst should also discuss analytical procedures, detection limits, and data delivery schedules with analytical laboratory personnel. The Analyst is also responsible for keeping this procedure updated, for participating in nonconformance report activities (Reference 5), for conducting data analysis (Reference 6), reporting results (Reference 7 ) and for communicating changes, as needed, to other EMS staff members.

\section{EMS QUALTTY ASSURANCE COORDINATOR}

The QA Coordinator is responsible for keeping lists of all present and past sampling locations, evaluating the need for and reviewing/approving soil sampling method design changes (Reference 4), and for assigning new soil sampling location designators as needed (Reference 8). The QA Coordinator is also responsible for inspecting and evaluating sample collection methods and documentation (References 9 and 10), participating in nonconformance report activities (Reference 5), and for archiving all design changes and revisions of this procedure (Reference 11).

\section{EMS TECHNICIANS}

The EMS Technicians are responsible for collecting soil and sediment samples according to methods detailed in this procedure at locations described in Reference 2. They must complete appropriate Field Tracking Forms (FIF) and Chain-of-Custody Record Forms (COCs) and must make detailed notes (on FIFs) as pertinent information regarding soil and sediment samples is acquired (see References 8 and 10). In particular, they must record the distance and direction from permanent location markers to the actual sampling locations. Technicians are also responsible for providing input to the Analyst regarding the suitability of sampling locations and sampling methods, the availability of samples, and any problems associated with the monitoring networks (Reference 5). 


\begin{tabular}{|c|c|c|}
\hline $\begin{array}{c}\text { REVISION 3 } \\
\text { FINAL }\end{array}$ & $\begin{array}{c}\text { EMP-S-S } \\
\text { Soil \& Arroyo Sediment Sampling }\end{array}$ & $\begin{array}{c}\text { Effective date: 01/04/93 } \\
\text { Page 5 of } 16\end{array}$ \\
\hline
\end{tabular}

\section{PROCEDURE}

Equipment Required:

SURFACE SOIL SAMPLING

Stainless steel soil core sampler (custom fabricated by EMS Technicians; B-596)

Resin striking surface

Sledge hammer, 5 to $8 \mathrm{lb}$. (LLNL Stores Stock \#5120-26051)

Plastic sample collection bags (LLNL Stores Stock \#8105-27696)

Yellow tie-on sample tags (LLNL Stores Stock \#8135-27958)

Indelible marking pens (LLNL Stores Stock \#7520-62156)

Tape measure, $50 \mathrm{ft}$. (LLNL Stores Stock \#5185-27672)

Magnetic compass (LLNL Stores Stock \#6650-18471)

Plastic vial for soil subsample, Be analysis (LLNL Stores Stock \#8125-48618)

Adhesive labels (LLNL Stores Stock \#7530-46221)

Field Tracking Form

Chain-of-Custody Record Forms (2 each)

Ice chest (no ice required; Transportainer TY-165, end of 6th St., LLNL)

\section{ARROYO SEDMMENT SAMPLING}

Hand auger w/extensions (Environmental Instruments Co., Concord CA; B-596)

Split-spoon sampler (Environmental Instruments Co., Concord CA; B-596)

Stainless-steel tubes (Building 596)

Slide hammer (Building 596)

Pipe wrenches (2 each; LINL Stores Stock \#5120-26390)

Large screwdriver (LLNL Stores Stock \#5120-26241)

Ice chest $w /$ double-bagged ice (Transportainer TY-165, end of 6th St, LLNL)

Wide-mouth glass sample collection containers (LLNL Stores Stock \#8125-27800)

Adhesive labels (L.LNL Stores Stock \#7530-46221)

Indelible markers (LLNL Stores Stock \#7520-62156)

Teflon sheets, 4 inches by 4 inches (LLNL Pipe Shop, B-511)

Protective caps (LLNL Pipe Shop, B-511)

Duct tape

Tape measure, $50 \mathrm{ft}$. (LLNL Stores Stock \#5185-27672)

Wooden stakes (LLNL Carpenter Shop, B-511)

Trowel

Field Tracking Form

Chain-of-Custody Record Form

\section{DECONTAMINATION AND PERSONAL PROTECTIVE EQUTPMENT}

Disposable vinyl gloves (LLNL Stores Stock \#8415-64995) .

Deionized water and detergent (LLNL Stores Stock \#7930-30299)

Large test tube brush (LLNL Stores Stock \#7920-30233)

Large rinse tray (photo developing, LLNL Stores Stock \#6750-29166),

Kimwipes or equivalent (LLNL Stores Stock \#7999-63447)

Garbage bags (LENL Stores Stock \#8105-58929)

Leather protective gloves (LLNL Stores Stock \#8415-50328)

Safety shoes (boots; LLNL Supervisor controlled)

Safety glasses (LLNL Stores Stock \#4270-34827) 


\begin{tabular}{|c||c|c|}
\hline $\begin{array}{c}\text { REVISION 3 } \\
\text { FINAL }\end{array}$ & $\begin{array}{c}\text { EMPP-S-S } \\
\text { Soil \& Arroyo Sediment Sampling }\end{array}$ & $\begin{array}{c}\text { Effective date: 01/04/93 } \\
\text { Page 6 of 16 }\end{array}$ \\
\hline
\end{tabular}

All soil and sediment samples must be collected according to the methods described in this procedure. All sampling activities must take place at designated sampling locations (Reference 2). FTFs and COCs must be completed for all samples, in accordance with References 8 and 10.

\subsection{SURFACE SOIL SAMPLING (LIVERMORE VALLEXISTTE 300 )}

The soil sampling methods are identical for the Livermore Valley and Site 300 soil monitoring networks. Site 300 access and safety procedures must be strictly followed when performing all sampling activities (Reference 12). Soil samples shall be collected annually, usually in the months of April and May. The collection of surface soils requires only nominal personal protective equipment (disposable vinyl gloves, safety glasses, safety boots).

\subsection{SELECTION OF SOIL SAMPLE MATERIAL}

6.1.1.1. Locate the permanent soil sample location marker (red and white fence post with metal identification tag attached; Reference 2). Select an undisturbed area, free of rocks and not sheltered by trees or buildings, in the vicinity of the permanent location marker.

- 6.1.1.2. Choose a $1 \mathrm{~m}^{2}$ area from which to collect the sample. The sample will consist of five subsamples, collected at the four corners and the center of the square. If a $1 \mathrm{~m}^{2}$ area is not available (such as along a roadside or a fence) the sample will be collected in a line, with each subsample being taken $1 \mathrm{~m}$ apart.

6.1.1.3. Using the tape measure and the compass for measurements, note the distance and direction of the actual sampling location from the permanent identification marker (Reference 2). Record this distance and direction on the FTF.

\subsection{SOIL SAMPLE VOLUME}

The total sample should weigh between 0.5 and $1.0 \mathrm{~kg}$. A $10 \mathrm{~g}$ aliquot is taken from the sample for beryllium analysis.

\subsubsection{OBTAINING THE SOIL SAMPLE}

Note: Prior to the actual collection of the samples, it is vital that the sampler be aware of EMS sampling hygiene. This includes wearing disposable gloves when collecting samples and discarding the gloves between locations. Take special precautions not to introduce any external contamination. This includes keeping the work area clean.

6.1.3.1. If necessary, carefully clip surface vegetation at the soil surface from the five subsampling areas. Do not disturb the soil surface.

6.1.3.2. Place the clean, decontaminated core sampler, resin striking surface at the top, on the first subsample site. With a sledge hammer, drive the core sampler into the ground until the ground surface is level with the bottom of the steel band welded to the outside of the core sampler. (At some locations, the soil may be loose enough so you can merely twist the core sampler into the ground by hand.)

6.1.3.3. Remove the resin striking surface. Twist the core sampler in order to break off the core it contains. If the soil sample is loose within the 


\begin{tabular}{|c|c|c|}
\hline $\begin{array}{c}\text { REVISION 3 } \\
\text { FINAL }\end{array}$ & $\begin{array}{c}\text { EMP-S-S } \\
\text { Soil \& Arroyo Sediment Sampling date: 01/04/93 } \\
\text { Page 7 of } 16\end{array}$ \\
\hline
\end{tabular}

corer, capture the soil sample by slipping a newly gloved hand under the base of the core sampler as it is removed. Be careful not to lose or contaminate any part of the sample. Should loss of subsample integrity occur, the subsample shall be discarded and another shall be collected.

6.13.4. Shake the contents of the core sampler into the plastic sample collection bag. If the sample is not easily shaken out, place two plastic bags over the handle of the sledge hammer and gently tap the core sample into the sample collection bag.

6.1.3.5. Repeat the collection steps 6.1.3.1 through 6.1.3.4 at the four remaining subsample sites, combining the five subsamples into the same sample bag.

6.1.3.6. Make sure the subsamples are adequately mixed. Then obtain an approximately $10 \mathrm{~g}$ aliquot from the sample and place it in a plastic vial. Place a white, self-stick label on the vial (Attachment A). Record the following information on the label: the sample identifier (including sample location designator), the type of sample, the date and year of collection, the type of analysis required (Be), the analytical laboratory, "LLNL", and the collector's initials.

6.1.3.7. Secure the sampling collection bag with a rubber band. Mark the outside of the bag with the sampling location using a waterproof, indelible marking pen. Place this bag inside another plastic bag and secure the second bag with a rubber band. Attach a yellow sample identification tag to the outside bag (Attachment B). The tag shall contain, at a minimum, the sample identifier (including sample location designator), the type of sample, the date and year of collection, the analyses required (gamma, plutonium), the analytical laboratory, and the collectors initials.

\subsubsection{SOIL SAMPLE FIELD DOCUMENTATION}

Record the appropriate information on the FTF (References 2 and 10). Be sure to record the distance and direction from the permanent sampling location to the location at which the sample was obtained. Record any other pertinent information about the sample.

\subsubsection{SOIL SAMPLE PRESERVATION}

No special preservation measures are required for soil samples. However, samplers must ensure the integrity of sample containers to avoid crosscontamination.

\subsubsection{SOIL QUALTYY ASSURANCE SAMPLES}

QA samples will be submitted with each batch of samples, in accordance with Reference 13. Duplicate samples are the only QA samples authorized for the soil sampling networks. Two duplicate samples are required for the Livermore Valley soil sampling network and two duplicate samples are required for the Site 300 soil sampling network. At locations chosen for duplicate samples, two samples are to be collected. Each sample will consist of five subsamples. At each corner and at the center of the sampling square, two cores will be collected and processed separately. Duplicate $10-g$ sample aliquots for beryllium analyses will also be produced. The sampling locations of QA samples are not to be identified on the bags, sample identification tags, or vial labels (References 8 and 10). However, this information is required on FTFs, and the QA sample must be assigned a temporary identification number (References 8 and 10 ). 


\begin{tabular}{|c|c|c|}
\hline $\begin{array}{c}\text { REVISION 3 } \\
\text { FINAL }\end{array}$ & $\begin{array}{c}\text { EMP-S-S } \\
\text { Soil \& Amoyo Sediment Sampling }\end{array}$ & $\begin{array}{c}\text { Effective date: 01/04/93 } \\
\text { Page 8 of 16 }\end{array}$ \\
\hline
\end{tabular}

\subsubsection{SOIL SAMPLING EQUIPMENT DECONTAMINATION}

Gloves must be discarded after collecting each sample, and must not be reused. After all subsamples are collected from a location, decontaminate the core sampler by washing it thoroughly with deionized water and a low-phosphate detergent. The large test tube brush should be used to clean the inside of the corer and to remove any particles adhering to the outside. The core sampler must then be thoroughly rinsed with deionized water. Rinse any other equipment that has come into contact with soil, using the same procedures. Dry the core sampler with a Kimwipe and place it in a plastic bag to prevent contamination.

\subsubsection{CHAIN OF CUSTODX}

Chain-of-custody procedures (Reference 10) must be followed. After all samples have been collected from a soil network, use the information from the FTF to complete a COC for each analytical laboratory. In addition to sample identification information, each COC must specify the laboratory name and the analyses to be completed.

\subsubsection{SOIL SAMOPLE DELTVERY ,}

Deliver the samples in the plastic sampling bags to the Radiation Analytical Sciences Laboratory (RASL). Building 151, Room 2143. If the samples cannot be delivered on the day of collection, they must be kept secure (Reference 10) until they can be delivered. Samples going to a contract analytical laboratory for beryllium analyses must be delivered, in accordance with Reference 15, to the sample courier. Chain-of-custody procedures must be followed whenever samples are delivered to laboratory personnel or to couriers.

Plutonium content of samples is determined by alpha spectroscopy of sample aliquots. Other sample aliquots are analyzed by gamma spectroscopy using a Ge(Li) detector (Reference 16). Beryllium content is determined by graphitefurnace atomic absorption spectroscopy.

\subsubsection{SOIL DATA REVIEW, ANALYSIS, MANAGEMENT, ARCHIVING}

The Analyst will review and analyze data promptly after receipt, in accordance with Reference 6 . Chain-of-custody procedures will be followed for data (Reference 10), and QA samples will be identified by the Analyst when signing and retuming COCs, following receipt of data, to the analytical laboratory. Data will be given to the Data Manager promptly for entry into the EMS Monitoring Database (References 10 and 14), and will be archived according to Reference 11.

\subsubsection{SOIL DATA REPORTING}

The monitoring results will be reported in the Site Annual Environmental Report (Reference 7).

\subsection{ARROYO SEDIMENT SAMPLING}

Arroyo sediment samples shall be collected annually, during the months of June and July, after seasonal rains have ceased. The collection of sediment samples requires the use of personal protective equipment (disposable vinyl gloves, leather gloves, safety glasses, safety shoes). An individual sediment sample is collected as two subsamples, of varying depths, in two steps. The first subsample is collected with the hand auger and is submitted for radiological analyses. The second subsample is 


\begin{tabular}{|c|c|c|}
\hline $\begin{array}{c}\text { REVISION 3 } \\
\text { FINAL }\end{array}$ & $\begin{array}{c}\text { EMP-S-S } \\
\text { Soil \& Arroyo Sediment Sampling }\end{array}$ & $\begin{array}{c}\text { Effective date: 01/04/93 } \\
\text { Page 9 of 16 }\end{array}$ \\
\hline
\end{tabular}

collected from the bottom of the same hole, using the split-spoon sampler, and is analyzed for metals and organic compounds.

Note: The sediment sampling procedure involves the use of
heavy equipment. Care should be exercised to avoid injury
when using the sampling equipment. The normally high
daytime temperatures during the sampling period, combined
with the strenuous physical activity required to operate the
slide hammer and auger, make it mandatory that a team
consisting of no less that three qualified persons perform the
sampling.

\subsection{SELECTION OF ARROYO SEDIMENT SAMPLE MATERIAI}

6.2.1.1. Locate the permanent sediment sampling location marker at the sampling location. A culvert/bridge or other permanent marker, described in Reference 2, serves as a reference point for that sampling location. Drive a wooden stake into the ground at the permanent location marker.

6.21.2. The Analyst will have supplied three random numbers on the Field Tracking Form. Samples are to be collected at distances (in feet) from the permanent marker corresponding to those numbers. Using the tape measure, measure from the permanent sampling location marker and place stakes at the sampling locations. Samples should be collected from the middle of the drainage channel at the most "down stream" number first, working upstream for each successive sample.

\section{ARROYO SEDIMENT SAMPLE VOLUME}

Sediment samples are obtained with various devices. The auger should provide enough sample to fill the glass sample container that will be submitted to RASL. The split-spoon sampler is designed to place samples directly into sample containers, which are merely sealed and sent to the analytical laboratory.

\section{OBTAINING THE ARROYO SEDIMENT SAMPLE}

Note: Prior to the actual collection of the samples, it is vital that the sampler be aware of EMS sampling hygiene. This includes wearing disposable gloves when collecting samples and discarding the gloves between samples. Take special precautions not to introduce any external contamination. This includes keeping the work area clean.

6.2.3.1. Remove any loose impediments from the sediment surface at the exact location where the sample is to be collected.

6.2.3.2. Using the hand auger, dig to a depth of $1 \mathrm{ft}$ from ground level. Remove and discard all loose sediment from the hole and the auger. Once the hole and auger are clean, continue to dig to a total depth of 1.5 feet.

6.2.3.3. Remove the auger from hole and place the sediment that is attached to the auger bit into a glass sample container. In the event that the sediment is dry and will not remain attached to the auger, use a trowel to fill the glass sample container with sediment from the hole. 


\begin{tabular}{|c|c|c|}
\hline $\begin{array}{c}\text { REVISION 3 } \\
\text { FINAL }\end{array}$ & $\begin{array}{c}\text { EMP-S-S } \\
\text { Soil \& Arroyo Sediment Sampling }\end{array}$ & $\begin{array}{c}\text { Effective date: 01/04/93 } \\
\text { Page 10 of 16 }\end{array}$ \\
\hline
\end{tabular}

6.2.3.4. Using the indelible marking pen, prepare an adhesive label. The label should include the sample identifier (including the sample location designator), the sample collector's initials, the type of sample, the analyses required (tritium, gamma), the analytical laboratory (RASL), and the sampling date (Attachment $C$. Secure the lid to the jar with duct tape, attach the label to the jar, and place the sample container into the ice chest. This portion of the sample goes to RASL for tritium and other radiological analyses, and must be kept cool prior to analyses.

6.23.5. Clear all loose sediment from the hole to prepare for collecting the second subsample, which will be collected using the split-spoon sampler.

6.23.6. Place two clean, decontaminated stainless-steel tubes into the split-spoon sampler, and assemble the sampler/slide hammer.

623.7. Place the sampler/slide hammer into the hole. Use the slide hammer to drive the sampler into the ground far enough to fill the bottom six inch tube (usually about eight to ten inches).

6.2.3.8. Remove the sampler from the hole by rotating the sampler, and disassemble the split-spoon sampler.

6.23.9. With freshly gloved hands, remove the bottom tube, making sure that the sediment sample is flush with both ends of the tube. Place a square Teflon sheet over each end of the sample tube and cover the Teflon sheets with plastic caps. Tape the plastic caps to the sample tube (use duct tape; do not use vinyl tapes, because they contain volatile organic compounds, which can contaminate the sample).

6.2.3.10. Using the indelible marking pen, prepare an adhesive label. The label should include the sample identifier (including the sample location designator), the sample collector's initials, the type of sample (sediment), the analyses required (CAM metals, EPA 8010, EPA 8020, EPA 8080), the analytical laboratory (usually a contract laboratory), "LLNL", and the sampling date (Attachment D). Attach the label to the sample tube and place the tube into the ice chest.

\subsection{ARROYO SEDIMENT SAMPLE FIELD DOCUMENTATION}

Record appropriate information on the FTF (References 2 and 10). Record any pertinent information about the sample.

\subsubsection{ARROYO SEDIMENT SAMPLE PRESERVATION}

All sediment samples must be kept cool until analysis, and must be kept on ice while in the field. Upon returning to LLNL, samples for radiological analyses that are in glass sample containers should be kept in a refrigerator, and sediment samples for metals and organic analyses that are in the stainless-steel tubes should be kept in a freezer. The samples must still be refrigerated/frozen when delivered to analytical laboratories.

\subsection{ARROYO SEDIMENT QUALTYY ASSURANCE SAMPLES}

QA samples are to be submitted with each batch of samples, in accordance with Reference 13 . In reality, the three samples taken at each sediment sampling locations are duplicate samples. However, blind duplicates are also required. A fourth sample is to be taken from each of three designated sampling locations and submitted blind, as a QA sample, to analytical laboratories. For locations chosen for duplicate sampling, a fourth random number is generated and a sample is collected using identical methods. The sampling locations of QA samples are not to 


\begin{tabular}{|c||c|c|}
\hline $\begin{array}{c}\text { REVISION 3 } \\
\text { FINAL }\end{array}$ & EMP-S-S & $\begin{array}{c}\text { Effective date: 01/04/93 } \\
\text { Page 11 of 16 }\end{array}$ \\
\hline
\end{tabular}

be identified on the sample containers or COCs (References 8 and 10). However, this information is required on FTFs.

\subsubsection{ARROYO SEDIMENT SAMPLING EQUIPMENT DECONTAMINATION}

Gloves must be discarded after collecting each sample, and must not be

reused. Before collecting additional samples, either at the same location or at another location, the auger, split-spoon sampler, and other sampling equipment must be decontaminated.

Wash the equipment thoroughly with deionized water and and a lowphosphate detergent. The large test tube brush should be used to clean the inside of the samplers and to remove any sediment adhering to the outside of the samplers. Rinse all equipment thoroughly with deionized water. Dry the samplers with Kimwipes and place the samplers in a plastic bag to prevent subsequent contamination.

\subsection{CHANN OF CUSTODY}

Chain-of-custody procedures (Reference 10) must be followed. Using information from the FTF, complete the COCs after all samples have been collected from the arroyo sediment sampling network. Two COCs are required for each set of samples because the subsamples are sent to different laboratories for processing. In addition to sample identification information, the COC must specify the laboratory name and the analyses required. Be sure to include the $C O C$ numbers on the FTF as a cross-reference.

\subsection{ARROYO SEDIMENT SAMPLE DELIVERY}

On the same day that they are collected, deliver the radiological subsamples to the RASL, Building 151, Room 2143. If the samples cannot be delivered on the day of collection, they must be kept secure (Reference 10) and refrigerated until they can be delivered. Chain-of-custody procedures must be followed when delivering samples (Reference 10).

Tritium content is determined by liquid scintillation counting of water recovered from the samples by using freeze-drying techniques (lyophilization). Plutonium content is determined by alpha spectroscopy, and other radionuclides are determined by gamma spectroscopy (Reference 16).

Samples submitted for organic analyses have "holding times," (the period following sampling in which analyses must be completed) so timely processing and submittal of sediment samples is essential. Frozen subsamples contained in sampling tubes are to be sent to analytical laboratories via courier as soon as possible, preferably on the day of collection (Reference 15). Chain-of-custody and sample-preservation procedures must be observed.

The sediment samples are analyzed for metals by inductively-coupled plasma emission spectroscopy (ICP). The samples are analyzed for organic compounds by using standard EPA methods $(8010$ for halogenated volatile organic compounds, method 8020 for aromatic volatile organic compounds, and method 8080 for pesticides and PCBs).

\subsection{SEDIMENT DATA REVIEW, ANALYSIS, MANAGEMENT, ARCHIVING}

The Analyst will review and analyze data promptly after receipt, in accordance with Reference 6 . Chain-of-custody procedures will be followed for data (Reference 10), and QA samples will be identified by the Analyst when signing and retuming $\mathrm{COCs}$, following receipt of data, to the analytical laboratory. Data will be given to the Data Manager promptly for entry into the EMS 


\begin{tabular}{|c|c|c|}
\hline $\begin{array}{c}\text { REVISION 3 } \\
\text { FINAL }\end{array}$ & EMP-S-S & $\begin{array}{c}\text { Effective date: 01/04/93 } \\
\text { Page 12 of } 16\end{array}$ \\
\hline
\end{tabular}

Monitoring Database (References 10 and 14), and will be archived according to Reference 11.

\section{SEDIMENT DATA REPORTING}

. The sediment sampling results will be reported in the Site Annual Environmental Report (Reference 7).

\section{DOCUMENTATION}

Samplers must complete FTFs and COCs when collecting soil and arroyo sediment samples, in accordance with Reference 10. FTFs must contain current location designators (Reference 8). FIFs and COCs become QA records and must be archived in the Document Retention Center, along with final sample data (Reference 11). Any deviations from this procedure must be documented in Nonconfomance Reports (Reference 5). Any changes to this procedure must be documented in an approved Design Change Package (Reference 4). All Nonconformance Reports and Design Change Packages are QA records and must be archived (Reference 11).

\section{ACCEPTANCE CRITERIA}

Only samples taken at official sampling locations will be used in the assessments of LLNL impacts on the environment, unless special samples are authorized in an approved sampling plan. Samples will not be deemed acceptable if the proper collection and preservative methods have not been followed, if the chain-of-custody has been broken or compromised, if the documentation has not been properly completed, or if the sample label or tag is illegible. Samples must be identified with authorized location designators (References 8 and 10) and documentation must include pertinent information as required by this procedure. Furthermore, samples will not be deemed acceptable if the sample containers are not properly labeled, dated, and initialed by the sample collector, if sample contamination is suspected, or if sampling schedules have not been met.

\section{ATTACHMENTS}

A. Soil Sample Label (Beryllium Analysis)

B. Soil Sample Tag

C. Arroyo Sediment Sample Label (Radiological Analyses)

D. Arroyo Sediment Sample label (Metals/Organic Compounds Analyses) 


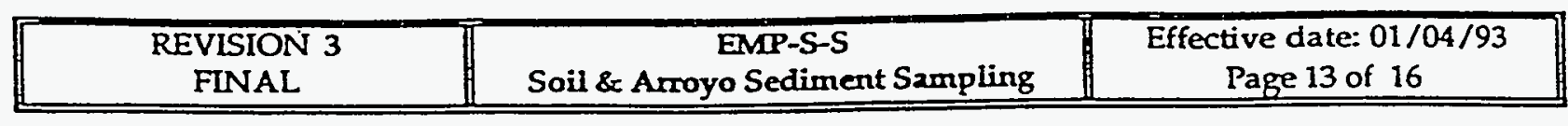

ATTACHMENT A. SOIL SAMPLE LABEL (BERYLLIUM ANALYSIS)

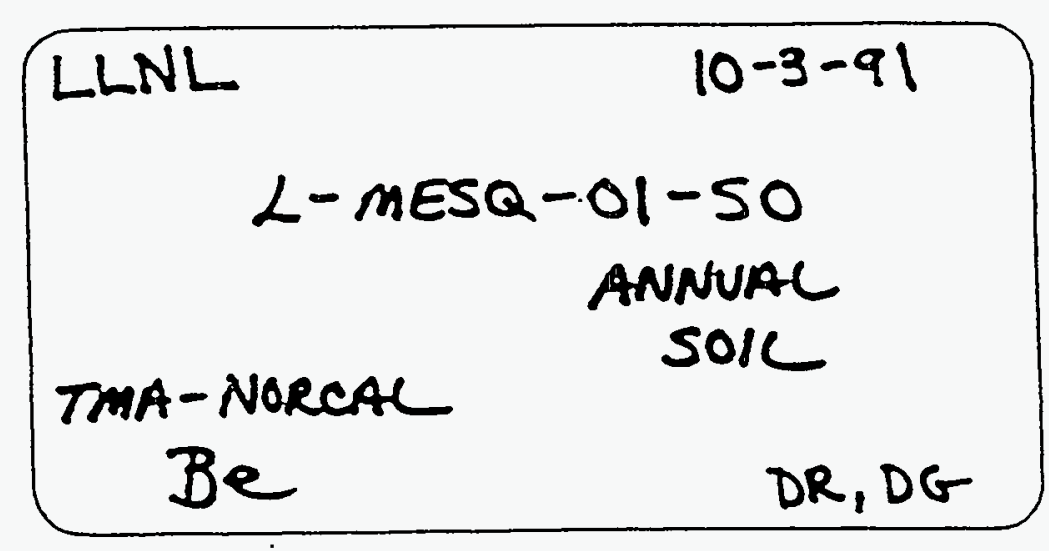

C-13 

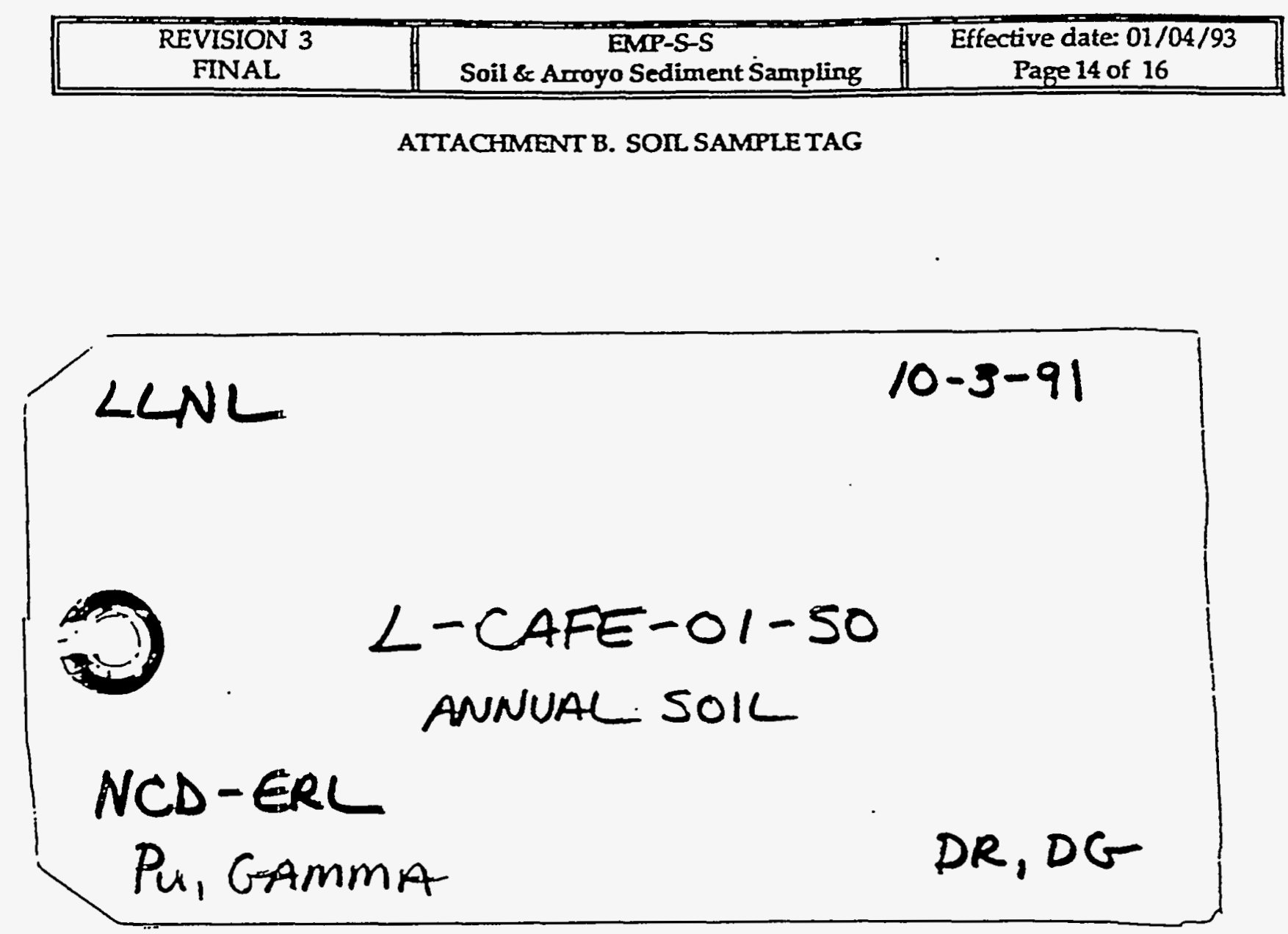

c-14 


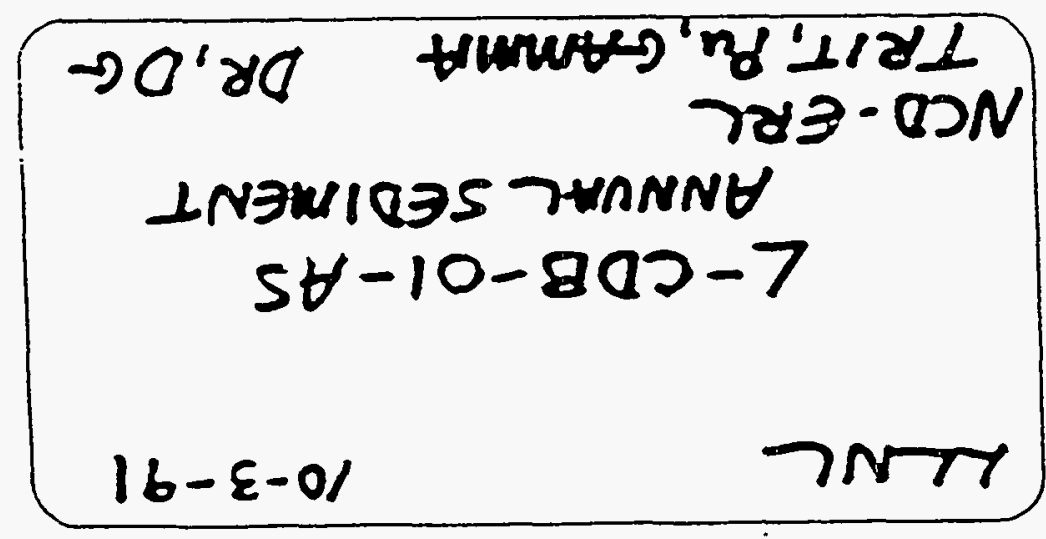

(SASXTVNV TVDIDOIOIAYY)

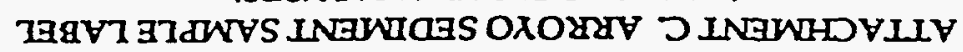

\begin{tabular}{|c|c|c|}
\hline 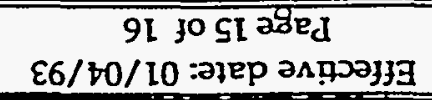 & 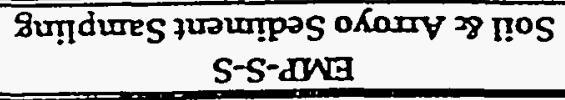 & 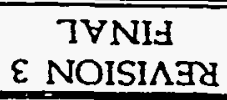 \\
\hline
\end{tabular}


ATTACHMENT D. ARROYO SEDIMENT SAMPLE LABEL (METALS/ORGANIC COMPOUNDS ANALYSES)

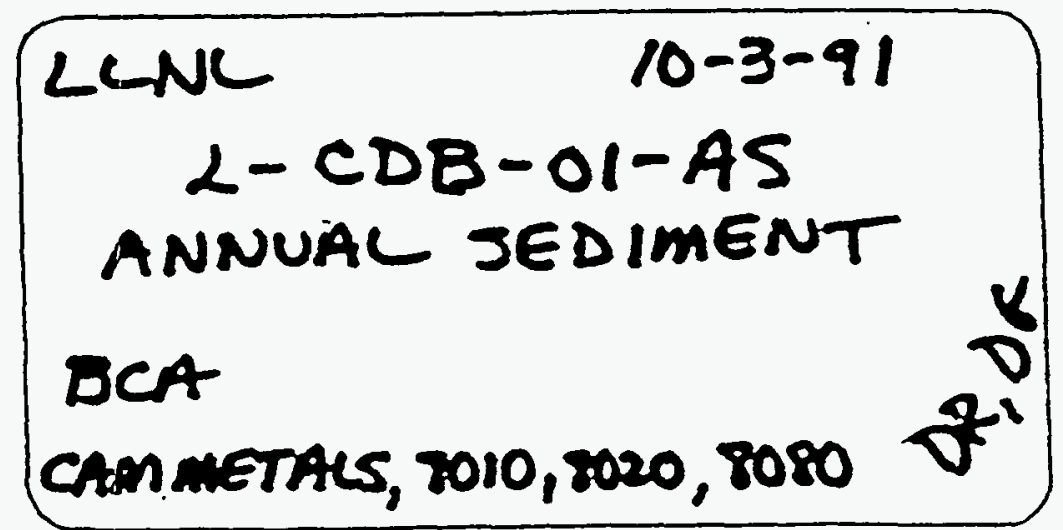




\section{Appendix D}

Analytical Results from 1991 Sediment Study 


\section{Appendix D \\ Analytical Results from 1991 Sediment Study}

Table D-1. Soluble metals analysis from 1991 sediments study (mg/L).

\begin{tabular}{|c|c|c|c|c|c|c|c|}
\hline Locations & Barium & Cadmium & Copper & Lead & Nickel & Vanadium & Zinc \\
\hline \multicolumn{8}{|l|}{ Catch basin } \\
\hline CB-140J-13 & 5.4 & 0.08 & 1.1 & 4.5 & 0.4 & 0.23 & 30 \\
\hline CB-170B-18 & 4.4 & $\leq 0.05$ & & 0.6 & 0.4 & & \\
\hline CB-190Q-10 $1 / 2$ & 5.4 & 0.1 & 2.2 & 2.4 & 0.4 & 0.22 & 30 \\
\hline CB-270G-29 & & $\leq 0.05$ & 0.88 & 3.5 & 0.5 & & 45 \\
\hline CB-290A-96 & 3.7 & $\leq 0.05$ & & 0.8 & 0.5 & & 48 \\
\hline CB-290F-38 & 7.4 & 0.12 & 1.4 & 1.9 & 1 & 0.65 & \\
\hline CB-290J-82 & & $\leq 0.05$ & 0.47 & 1.2 & & & 17 \\
\hline CB-290N-19 & & 0.1 & 0.39 & 1.5 & & & 78 \\
\hline CB-350E-44 & 6.1 & 0.1 & 0.83 & 11 & 0.6 & & 80 \\
\hline CB-360G-20 1/2 & 5.6 & 0.14 & 7 & 2.4 & 1.7 & & 31 \\
\hline CB-360J-48 & 3.3 & $\leq 0.05$ & 0.48 & 2.5 & 0.4 & & 40 \\
\hline CB-410D-014 & 5.3 & 0.12 & & 3.7 & 0.6 & & 28 \\
\hline CB-410E-044 & 4 & 0.12 & 3.2 & 63 & 0.4 & 0.15 & 14 \\
\hline CB-420D-024 & 6.6 & 0.12 & 1.4 & 4.7 & 0.7 & & 15 \\
\hline CB-620J-01-34 & & 0.26 & 1.3 & 4.1 & 0.4 & & 26 \\
\hline CB-620J-34 & & 0.13 & 0.71 & 1.1 & 0.02 & & 8.5 \\
\hline Open channel & & & & & - & & \\
\hline OCS-150-1 & 8.1 & 0.17 & 1.9 & 3.6 & 0.7 & 0.48 & 82 \\
\hline OCS-170-1 & 8.9 & $\leq 0.05$ & & 0.6 & 0.7 & 0.24 & 30 \\
\hline OCS-180-1 & 8.6 & $\leq 0.05$ & & 0.5 & 0.9 & 0.34 & 18 \\
\hline OCS $-190-1$ & 8.1 & $\leq 0.05$ & 1.2 & 0.6 & 0.9 & 0.59 & \\
\hline OCs-190-2 & & $\leq 0.05$ & 5.7 & & & & \\
\hline OCS $-190-4$ & 7.2 & 0.05 & & 0.3 & 0.6 & 0.36 & \\
\hline OCS-290-1 & 7 & $\leq 0.05$ & & & 0.6 & 0.29 & \\
\hline OCS-290-2 & 5.2 & $\leq 0.05$ & 1.9 & 1.2 & 0.6 & 0.24 & \\
\hline
\end{tabular}

Table D-2. ICAP metals analysis from 1991 sediments study on liquid samples (mg/L).

\begin{tabular}{|c|c|c|c|c|c|c|c|c|c|c|c|c|c|c|c|c|c|}
\hline Locations & Sb & As & $\mathrm{Ba}$ & $\mathrm{Be}$ & Cod & $\mathrm{Cr}$ & Co & $\mathrm{Cu}$ & $\mathrm{Pb}$ & $\mathrm{Hg}$ & Mo & $\mathrm{Ni}$ & Se & $\mathrm{Ag}$ & Th & v & $\mathrm{Zn}$ \\
\hline CB-160C-30 & $\leq 0.2$ & 0.007 & 0.46 & & $\leq 0.05 \leq$ & $\leq 0.05 \leq$ & $\leq 0.05$ & 1.6 & $\leq 0.2$ & 0.01 & $\leq 0.2 \leq$ & & & & & & $3.4^{\circ}$ \\
\hline MH-340A-71 & $\leq 0.2$ & 0.043 & 0.08 & $\leq 0.01$ & $\leq 0.05 \leq$ & $\leq 0.05 \leq$ & $\leq 0.05 \mathrm{C}$ & 0.025 & $\leq 0.2 \leq$ & $\leq 0.0005$ & $\leq 0.2 \leq$ & $\leq 0.1 \leq$ & $\leq 0.002$ & $\leqslant 0.05$ & $\leq 0.2 \leq$ & $\leq 0.05$ & 0.38 \\
\hline CB-190T-49 & $\leq 0.2$ & 0.006 & 11 & 0.01 & 0.45 & 1.4 & 0.27 & 5.4 & 15 & 0.073 & $\leq 0.2$ & $1.2 \leq c$ & $\leq 0.002$ & $\leq 0.05$ & $\leq 0.2$ & 1.1 & 62 \\
\hline CB-290M-26 & $\leq 0.2$ & $\leqslant 0.002$ & 0.07 & $\leq 0.01 \leq$ & $\leq 0.05 \leq$ & $\leq 0.05 \leq$ & $\leq 0.05$ & 1.6 & $\leq 0.2$ & 0.032 & $\leq 0.2 \leq$ & $\leq 0.1 \leq$ & $\leq 0.002$ & $\leq 0.05$ & $5 \leq 0.2 \leq$ & $\leq 0.05$ & 1.9 \\
\hline CB-390Y-84 & & & & & 0.47 & 1.4 & 1.2 & 2.1 & 2.2 & 0.054 & $\leq 0.2$ & 1.4 & 0.01 & $\leq 0.05$ & 50.2 & 1.9 & 17 \\
\hline CB-130Y-24 & $\leq 0.2$ & 0.006 & $\leq 0.05$ & $\leq 0.01$ & $\leq 0.05$ & $0.05 \leq$ & $\leq 0.05$ & 0.09 & $\leq 0.2 \leq$ & $\leq 0.0005$ & & $\leq 0.1 \leq$ & $\leq 0.002$ & $\leq 0.05$ & & $\leq 0.05$ & 0.24 \\
\hline CB-220A-33 & & & & & $\leq 0.05 \leq$ & $\leq 0.05 \leq$ & $\leq 0.05$ & 0.26 & 0.2 & 0.0009 & $\leq 0.2 \leq$ & $\leq 0.1 \leq$ & $\leq 0.002$ & $\leq 0.05$ & $0.2 \leq$ & $\leq 0.05$ & 0.72 \\
\hline
\end{tabular}


Table D-3. ICAP metals analysis from 1991 sediments study on solid samples (mg/kg).

\begin{tabular}{|c|c|c|c|c|c|c|c|c|c|c|c|c|c|c|c|c|}
\hline Locations & $\mathrm{Sb}$ & As & $\mathrm{Ba}$ & $\mathrm{Be}$ & Cd & $\mathrm{Cr}$ & Co & $\mathrm{Cu}$ & $\mathrm{Pb}$ & $\mathrm{Hg}$ & Mo & $\mathrm{Se}$ & $\mathrm{Ag}$ & Th & V & $\mathrm{Zn}$ \\
\hline \multicolumn{17}{|l|}{ Background } \\
\hline BS-1-01-6 & $\leq 4$ & 1.4 & 170 & 0.4 & 7 & 28 & 8 & 15 & 6 & $\leq 0.05$ & $\leq 4$ & $32 \leq 0.4$ & $\leq 1$ & $\leq 4$ & 39 & 30 \\
\hline BS-1-02-6 & $\leq 4$ & 1.8 & 200 & 0.4 & 7 & 28 & 10 & 17 & $\leq 4$ & $\leq 0.05$ & $\leq 4$ & $32 \leq 0.4$ & $\leq 1$ & $\leq 4$ & 40 & 27 \\
\hline BS-2-8 & $\leq 4$ & 1.7 & 260 & 0.3 & 6 & 20 & 5 & 31 & 6 & $\leq 0.05$ & $\leq 4$ & $16 \leq 0.4$ & $\leq 1$ & $\leq 4$ & 28 & 49 \\
\hline BS-3-6 & $\leq 4$ & 1.8 & 210 & 0.4 & 6 & 18 & 7 & 28 & 11 & $\leq 0.05$ & $\leq 4$ & $18 \leq 0.4$ & $\leq 1$ & $\leq 4$ & 33 & 32 \\
\hline$B S-4-6$ & $\leq 4$ & 3.7 & 260 & 0.6 & 13 & 48 & 10 & 24 & 8 & 0.05 & $\leq 4$ & $39 \leq 0.4$ & $\leq 1$ & $\leq 4$ & 52 & 57 \\
\hline BS-5-6 & & & 520 & & 15 & 64 & 10 & 160 & 320 & 0.12 & 9 & $62 \leq 0.4$ & $\leq 1$ & $\leq 4$ & 77 & 290 \\
\hline$B S-6-6$ & $\leq 4$ & 1.9 & 310 & 0.5 & 14 & 75 & 15 & 31 & 30 & 0.14 & $\leq 4$ & $110 \leq 0.4$ & $\leq 1$ & $\leq 4$ & 53 & 80 \\
\hline BS7-6 & $\leq 4$ & 3.8 & 130 & 0.2 & 10 & 37 & 9 & 15 & 13 & $\leq 0.05$ & $\leq 4$ & $39 \leq 0.4$ & $\leq 1$ & $\leq 4$ & 28 & 60 \\
\hline \multicolumn{17}{|l|}{ Catch basin } \\
\hline CB-120M-21 & $\leq 4$ & 3.5 & 90 & $\leq 0.2$ & 11 & 36 & 4 & 99 & 120 & 0.6 & $\leq 4$ & $25 \leq 0.4$ & 10 & 2 & 18 & 1800 \\
\hline CB-120S-01-17 & $\leq 4$ & 7.9 & 110 & 0.3 & 7 & 43 & 7 & 120 & 69 & 0.73 & 4 & $26 \leq 0.4$ & 5 & $\leq 4$ & 22 & 430 \\
\hline CB-120S-02-17 & $\leq 4$ & 10 & 140 & 0.2 & 7 & 31 & 5 & 46 & 47 & 0.64 & $\leq 4$ & $18 \leq 0.4$ & 21 & $\leq 4$ & 20 & 280 \\
\hline CB-140J-13 & $\leq 4$ & 16 & 140 & 0.3 & 8 & 44 & 11 & 37 & 43 & 0.37 & $\leq 4$ & $43 \leq 0.4$ & $\leq 1$ & $\leq 4$ & 34 & 460 \\
\hline CB-150F-18 & & & 250 & & 14 & 39 & 12 & 24 & 16 & $\leq 0.05$ & $\leq 4$ & $41 \leq 0.4$ & $\leq 1$ & $\leq 4$ & 46 & 65 \\
\hline CB-150U-21 & $\leq 4$ & 1.4 & 76 & $\leq 0.2$ & 4 & 14 & 4 & 15 & 38 & 0.19 & $\leq 4$ & $15 \leq 0.4$ & $\leq 1$ & $\leq 4$ & 18 & 150 \\
\hline$C B-170 B-18$ & $\leq 4$ & 2.8 & 100 & 0.2 & 5 & 29 & 12 & 23 & 18 & 0.06 & $\leq 4$ & $43 \leq 0.4$ & 1 & $\leq 4$ & 24 & 140 \\
\hline CB-190Q-10 $1 / 2$ & $\leq 4$ & 2.8 & 110 & 0.2 & 6 & 38 & 8 & 52 & 23 & 0.37 & $\leq 4$ & $38 \leq 0.4$ & $\leq 1$ & $\leq 4$ & 31 & 390 \\
\hline CB-260G-38 & $\leq 4$ & 3.2 & 110 & 0.2 & 10 & 31 & 28 & 42 & 25 & 0.28 & $\leq 4$ & $39 \leq 0.4$ & $\leq 1$ & $\leq 4$ & 30 & 290 \\
\hline CB-270G-29 & $\leq 4$ & 1.4 & & & & & & & & $\leq 0.05$ & $\leq 4$ & $30 \leq 0.4$ & $\leq 1$ & $\leq 4$ & 10 & 280 \\
\hline CB-280D-24 & 120 & 2.9 & 86 & 0.2 & 8 & 60 & 10 & \multicolumn{2}{|c|}{42025000} & 0.24 & $\leq 4$ & $66 \leq 0.4$ & 6 & $\leq 4$ & 23 & 560 \\
\hline CB-290A-96 & $\leq 4$ & 1.4 & 110 & 0.2 & 4 & 36 & 6 & 15 & 17 & $\leq 0.05$ & $\leq 4$ & $29 \leq 0.4$ & $\leq 1$ & $\leq 4$ & 19 & 410 \\
\hline CB-290F-38 & 4 & 2.7 & 130 & 0.3 & 7 & 34 & 9 & 30 & 14 & $\leq 0.05$ & $\leq 4$ & $41 \leq 0.4$ & $\leq 1$ & $\leq 4$ & 29 & 220 \\
\hline CB-290J-82 & $\leq 4$ & 1.3 & 69 & $\leq 0.2$ & 3 & 23 & 6 & 28 & 30 & 0.06 & $\leq 4$ & $19 \leq 0.4$ & $\leq 1$ & $\leq 4$ & 16 & 250 \\
\hline CB-290N-19 & $\leq 4$ & 1.8 & 85 & $\leq 0.2$ & 4 & 19 & 8 & 29 & 22 & 0.09 & $\leq 4$ & $17 \leq 0.4$ & $\leq 1$ & $\leq 4$ & 20 & 810 \\
\hline CB-310C-24 & $\leq 4$ & 0.8 & 50 & $\leq 0.2$ & 5 & 340 & 6 & 13 & 570 & 0.07 & $\leq 4$ & $140 \leq 0.4$ & $\leq 1$ & $\leq 4$ & 9 & 240 \\
\hline CB-310M-30 & & & 180 & & 13 & 33 & 11 & 24 & 6 & $\leq 0.05$ & $\leq 4$ & $46 \leq 0.4$ & $\leq 1$ & $\leq 4$ & 40 & 120 \\
\hline CB-320-M & $\leq 4$ & 2.4 & 80 & $\leq 0.2$ & 7 & 62 & 8 & 68 & 76 & 0.07 & 10 & $69 \leq 0.4$ & $\leq 1$ & $\leq 4$ & 21 & 170 \\
\hline CB-320-M2 & $\leq 4$ & 2.5 & 100 & $\leq 0.2$ & 8 & 78 & 10 & 35 & 270 & 0.17 & 5 & $200 \leq 0.4$ & $\leq 1$ & $\leq 4$ & 21 & 140 \\
\hline CB-320F-17 & 5 & 3.9 & 120 & 0.2 & 22 & 97 & 15 & 290 & 400 & 38 & 15 & $91 \leq 0.4$ & 3 & $\leq 4$ & 20 & 3000 \\
\hline CB-340C-35 & $\leq 4$ & 2.6 & 110 & 0.2 & 4.7 & 32 & 9.4 & 17 & 4.8 & $\leq 0.05$ & $\leq 4$ & $44 \leq 0.4$ & $\leq 1$ & $\leq 4$ & 22 & 100 \\
\hline CB-350E-44 & $\leq 4$ & 2.7 & 100 & 0.2 & 5 & 28 & 8 & 73 & 34 & 0.1 & $\leq 4$ & $30 \leq 0.4$ & $\leq 1$ & $\leq 4$ & 16 & 560 \\
\hline CB-360G-20 $1 / 2$ & $\leq 4$ & 4.3 & 110 & 0.2 & 6 & 34 & 54 & 52 & 24 & 0.24 & $\leq 4$ & $59 \leq 0.4$ & $\leq 1$ & $\leq 4$ & 21 & 400 \\
\hline CB-360J-48 & $\leq 4$ & 3.6 & 110 & 0.2 & 5 & 120 & 9 & 34 & 100 & 0.05 & $\leq 4$ & $70 \leq 0.4$ & $\leq 1$ & $\leq 4$ & 23 & 940 \\
\hline CB-390R-14 1/2 & & & & & 9.2 & 28 & 9.6 & 96 & 36 & 0.13 & $\leq 4$ & $31 \leq 0.4$ & $\leq 1$ & $\leq 4$ & 21 & 390 \\
\hline$C B-410 D-014$ & $\leq 4$ & 2.3 & 160 & $\leq 0.2$ & 4 & 21 & 6 & 2.3 & 80 & 0.13 & $\leq 4$ & $27 \leq 0.4$ & $\leq 1$ & $\leq 4$ & 15 & 460 \\
\hline$C B-410 E-044$ & $\leq 4$ & 3.8 & 110 & 0.2 & 7 & 66 & 8 & 120 & 640 & $\cdot 1.3$ & $\leq 4$ & $54 \leq 0.4$ & $\leq 1$ & $\leq 4$ & 28 & 390 \\
\hline CB-420D-024 & $\leq 4$ & 0.9 & 150 & $\leq 0.2$ & 4 & 40 & 6 & 41 & 68 & 0.34 & $\leq 4$ & $44 \leq 0.4$ & $\leq 1$ & $\leq 4$ & 19 & 400 \\
\hline CB-420F-27 & $\leq 4$ & 3.1 & 94 & 0.2 & 9 & 93 & 12 & 44 & 120 & 0.35 & $\leq 4$ & $69 \leq 0.4$ & $\leq 1$ & $\leq 4$ & 29 & 710 \\
\hline CB-430T-86.5 & $\leq 4$ & 3.3 & 88 & $\leq 0.2$ & 3 & 21 & 3 & 19 & 24 & 0.3 & $\leq 4$ & $20 \leq 0.4$ & $\leq 1$ & $\leq 4$ & 15 & 440 \\
\hline$C B-460-C$ & $\leq 4$ & 3.9 & 160 & 0.3 & 8 & 45 & 10 & 25 & 15 & 0.1 & $\leq 4$ & $52 \leq 0.4$ & $\leq 1$ & 7 & 35 & 86 \\
\hline CB-470-P & $\leq 4$ & 3.2 & 130 & 0.2 & 9 & 35 & 10 & 19 & 7 & 0.08 & $\leq 4$ & $43 \leq 0.4$ & $\leq 1$ & $\leq 4$ & 30 & 240 \\
\hline CB-470-P2 & $\leq 4$ & 3.6 & 120 & 0.2 & 8 & 40 & 9 & 17 & 6 & 0.06 & $\leq 4$ & $44 \leq 0.4$ & $\leq 1$ & 5 & 31 & 150 \\
\hline$C B-490-X-36$ & $\leq 4$ & 0.05 & 76 & $\leq 0.2$ & 8 & 33 & 8 & 15 & 8 & & $\leq 4$ & $51 \leq 0.4$ & $\leq 1$ & 4 & 18 & 78 \\
\hline CB-510B-30 & $\leq 4$ & 3.9 & 70 & 0.2 & 3 & 33 & 3 & 18 & 25 & $\leq 0.05$ & $\leq 4$ & $24 \leq 0.4$ & $\leq 1$ & $\leq 4$ & 13 & 130 \\
\hline
\end{tabular}


Table D-3. ICAP metals analysis from 1991 sediments study on solid samples (mg/kg) (continued).

\begin{tabular}{|c|c|c|c|c|c|c|c|c|c|c|c|c|c|c|c|c|}
\hline Locations & Sb & As & $\mathrm{Ba}$ & $\mathrm{Be}$ & Cd & $\mathrm{Cr}$ & $\mathrm{Co}^{\circ}$ & $\mathrm{Cu}$ & $\mathrm{Pb}$ & $\mathrm{Hg}$ & Mo & Se & $\mathrm{Ag}$ & Th & v & $\mathrm{Zn}$ \\
\hline CB-520F-76 & & & 72 & 0.2 & 3 & 21 & 4 & 98 & 32 & & & & & & & \\
\hline CB-560G-14 & $\leq 4$ & 3.2 & 71 & $\leq 0.2$ & 5 & 61 & 8 & 29 & 15 & 0.11 & $\leq 4$ & $52 \leq 0.4$ & $\leq 1$ & $\leq 4$ & 17 & 150 \\
\hline CB-590-Q1-12 & $\leq 4$ & 0.05 & 160 & 0.2 & 8 & 37 & 7 & 49 & 44 & & $\leq 4$ & $32 \leq 0.4$ & $\leq 1$ & $\leq 4$ & 28 & 180 \\
\hline CB-610A-54 & $\leq 4$ & 43 & 240 & 0.3 & 11 & 29 & 9 & 26 & 54 & $\leq 0.05$ & $\leq 4$ & $43 \leq 0.4$ & $\leq 1$ & $\leq 4$ & 61 & 98 \\
\hline CB-610B-16 & $\leq 4$ & 2.5 & 100 & $\leq 0.2$ & 5 & 36 & 6 & 73 & 59 & $\leq 0.05$ & $\leq 4$ & $44 \leq 0.4$ & $\leq 1$ & $\leq 4$ & 21 & 210 \\
\hline CB-620J-01-34 & $\leq 4$ & 2.3 & 85 & $\leq 0.2$ & 6 & 22 & 7 & 48 & 64 & $\leq 0.05$ & 6 & $30 \leq 0.4$ & $\leq 1$ & $\leq 4$ & 14 & 420 \\
\hline CB-620J-34 & $\leq 4$ & 2.2 & 68 & $\leq 0.2$ & 6 & 27 & 5 & 30 & 38 & $\leq 0.05$ & $\leq 4$ & $30 \leq 0.4$ & $\leq 1$ & $\leq 4$ & 20 & 270 \\
\hline MH-110G-93 & $\leq 4$ & 3.6 & 130 & 0.2 & 10 & 67 & 11 & 480 & 110 & 5.5 & 6 & $50 \leq 0.4$ & 3 & 2 & 21 & 640 \\
\hline \multicolumn{17}{|l|}{ Open channel } \\
\hline$\overline{O C B}-620-28$ & $\leq 4$ & 2.5 & 38 & $\leq 0.2$ & 2 & 16 & 2 & 7 & 8 & $\leq 0.05$ & $\leq 4$ & $17 \leq 0.4$ & $\leq 1$ & $\leq 4$ & 9 & 140 \\
\hline ocs-10-1-6 & & & 180 & & 10 & 36 & 10 & 23 & 22 & 0.18 & $\leq 4$ & $40 \leq 0.4$ & $\leq 1$ & $\leq 4$ & 35 & 92 \\
\hline ocs-150-1 & $\leq 4$ & 7.3 & 160 & 0.4 & 9 & 43 & 12 & 50 & 80 & 1.2 & $\leq 4$ & $44 \leq 0.4$ & $\leq 1$ & $\leq 4$ & 38 & 1500 \\
\hline OCS-170-1 & $\leq 4$ & 4.6 & 180 & 0.4 & 7 & 39 & 12 & 23 & 53 & 0.1 & $\leq 4$ & $42 \leq 0.4$ & $\leq 1$ & $\leq 4$ & 36 & 400 \\
\hline OCS-180-1 & $\leq 4$ & 3.8 & 180 & 0.4 & 7 & 30 & 12 & 22 & 10 & $\leq 0.05$ & $\leq 4$ & $36 \leq 0.4$ & $\leq 1$ & $\leq 4$ & 37 & 280 \\
\hline OCS-190-1 & $\leq 4$ & 2.4 & 170 & 0.3 & 5 & 30 & 8 & 48 & 33 & 0.78 & $\leq 4$ & $26 \leq 0.4$ & $\leq 1$ & $\leq 4$ & 28 & 160 \\
\hline OCS-190-2 & $\leq 4$ & 3.7 & 87 & 0.2 & 3 & 15 & 5 & 30 & 4 & 0.2 & $\leq 4$ & $13 \leq 0.4$ & $\leq 1$ & 4 & 20 & 57 \\
\hline OCS-190-3-6 & $\leq 4$ & 3.9 & 330 & 0.7 & 12 & 47 & 13 & 27 & 7 & 0.06 & $\leq 4$ & $47 \leq 0.4$ & $\leq 1$ & $\leq 4$ & 54 & 62 \\
\hline OCS-190-4 & $\leq 4$ & 4.8 & 150 & 0.4 & 6 & 28 & 10 & 19 & 6 & $\leq 0.05$ & $\leq 4$ & $30 \leq 0.4$ & $\leq 1$ & $\leq 4$ & 34 & 130 \\
\hline OCS-280-1 & & & & & 10 & 33 & 10 & 23 & 16 & $\leq 0.05$ & $\leq 4$ & $42 \leq 0.4$ & $\leq 1$ & $\leq 4$ & 30 & 410 \\
\hline OCS-290-1 & $\leq 4$ & 2.2 & 130 & 0.3 & 5 & 27 & 11 & 15 & $\leq 4$ & $\leq 0.05$ & $\leq 4$ & $25 \leq 0.4$ & $\leq 1$ & 6 & 33 & 90 \\
\hline OCS-290-2 & $\leq 4$ & 3 & 130 & 0.3 & 5 & 27 & 10 & 54 & 18 & 0.37 & $\leq 4$ & $27 \leq 0.4$ & $\leq 1$ & $\leq 4$ & 30 & 160 \\
\hline $\operatorname{OCS}-460-1-6$ & & & & & 12 & 41 & 8.9 & 18 & 8 & $\leq 0.05$ & $\leq 4$ & $38 \leq 0.4$ & $\leq 1$ & $\leq 4$ & 41 & 77 \\
\hline OCS-490-1 & & & & & 7 & 21 & 8.2 & 14 & 6 & $\leq 0.05$ & $\leq 4$ & $32 \leq 0.4$ & $\leq 1$ & $\leq 4$ & 26 & 46 \\
\hline OCS $-490-2$ & & & & & 7.9 & 27 & 7.2 & 14 & $\leq 4$ & $\leq 0.05$ & $\leq 4$ & $25 \leq 0.4$ & $\leq 1$ & $\leq 4$ & 36 & 77 \\
\hline OCS-530-1-8 & $\leq 4$ & 0.44 & 130 & 0.2 & 10 & 66 & 9 & 20 & 13 & & $\leq 4$ & $48 \leq 0.4$ & $\leq 1$ & 4 & 28 & 120 \\
\hline OCS-540-1-12 & $\leq 4$ & 0.06 & 180 & 0.3 & 9 & 41 & 12 & 17 & .6 & & $\leq 4$ & $35 \leq 0.4$ & $\leq 1$. & $\leq 4$ & 40 & 52 \\
\hline OCS-590-1 & & & & & 7 & 20 & 7.4 & 9.6 & 6 & $\leq 0.05$ & $\leq 4$ & $19 \leq 0.4$ & $\leq 1$ & $\leq 4$ & 24 & $63^{\circ}$ \\
\hline OCS-590-2 & & & & & 4 & 15 & 6 & 7.2 & 4 & $\leq 0.05$ & $\leq 4$ & $15 \leq 0.4$ & $\leq 1$ & $\leq 4$ & 17 & 70 \\
\hline OCS-610-1-6 & $\leq 4$ & 4 & 220 & 0.4 & 10 & 38 & 14 & 19 & 16 & $\leq 0.05$ & $\leq 4$ & $39 \leq 0.4$ & $\leq 1$ & $\leq 4$ & 40 & 45 \\
\hline OCS-660-1-6 & $\leq 4$ & 50.05 & 150 & 0.2 & 8 & 50 & 10 & 14 & 24 & & $\leq 4$ & $70 \leq 0.4$ & $\leq 1$ & $\leq 4$ & 29 & 160 \\
\hline OCS-670-1-8 & 5 & 2 & 210 & 0.5 & 7 & 26 & 7 & 12 & 12 & 0.25 & $\leq 4$ & $23 \leq 0.4$ & $\leq 1$ & $\leq 4$ & 36 & 34 \\
\hline OCS-690-1-6 & $\leq 4$ & 2.4 & 250 & 0.3 & 10 & 20 & 6 & 42 & 5 & $\leq 0.05$ & $\leq 4$ & $22 \leq 0.4$ & $\leq 1$ & $\leq 4$ & 35 & 57 \\
\hline OCS-690-2-6 & 4 & 2 & 220 & 0.3 & 8 & 18 & 6 & 44 & 6 & $\leq 0.05$ & $\leq 4$ & $19 \leq 0.4$ & $\leq 1$ & $\leq 4$ & 27 & 51 \\
\hline OCS-690-2A-10 & & - & & & 10 & 35 & 9.2 & 14 & 7 & $\leq 0.05$ & $\leq 4$ & $30 \leq 0.4$ & $\leq 1$ & $\leq 4$ & 46 & 38 \\
\hline OCS-690-2B-10 & & & & & 13 & 43 & 7.5 & 18 & 5 & $\leq 0.05$ & $\leq 4$ & $27 \leq 0.4$ & $\leq 1$ & $\leq 4$ & 54 & 45 \\
\hline OCS-690-3-8 & & & & & 6 & 19 & 11 & 10 & 5 & $\leq 0.05$ & $\leq 4$ & $35 \leq 0.4$ & $\leq 1$ & $\leq 4$ & 22 & 35 \\
\hline
\end{tabular}


Table D-4. Radionuclide analysis for 1991 sediment study in $\mathrm{pCi} / \mathrm{g}$, unless otherwise noted.

\begin{tabular}{|c|c|c|c|c|c|c|c|c|c|}
\hline Locations & Gross $\alpha$ & Gross $\beta$ & $\begin{array}{c}{ }^{3} \mathrm{H} \\
(\mathrm{p} \mathrm{Ci} / \mathrm{L})\end{array}$ & $\begin{array}{c}\% \\
\text { moisture } \\
\end{array}$ & $40 \mathrm{~K}$ & ${ }^{137} \mathrm{Cs}$ & ${ }^{226} \mathrm{Ra}$ & ${ }^{228} \mathrm{Th}$ & ${ }^{232} \mathrm{Th}$ \\
\hline \multicolumn{10}{|l|}{ Background } \\
\hline BS-1-01-5 & 7 & 19 & 1302 & 5.6 & 11.84 & so.1924 & $\leq 0.7144$ & 1.099 & $\leq 0.9536$ \\
\hline & 7 & 15 & 881 & 5.5 & 12.09 & $\leq 0.1408$ & & 0.7008 & 0.9569 \\
\hline & $\leq 2$ & 16 & 245 & 5.2 & 5.62 & $\leq 0.06996$ & 0.5086 & 0.7361 & 0.5719 \\
\hline BS- & $\leq 3$ & 13 & $\leq-32$ & 1.6 & 8.99 & $\leq 0.1716$ & 0.4097 & 1.114 & 1.121 \\
\hline & 8 & 16 & 367 & 2.9 & 5.38 & $\leq 0.1734$ & 0.9029 & 1.028 & $\leq 1.288$ \\
\hline & $\leq 1$ & 16 & $\leq 32$ & 8.8 & 15.32 & $\leq 0.156$ & 0.5612 & 0.6171 & 0.9448 \\
\hline & 4 & 14 & 762 & 2.9 & .097 & $\leq 0.13$ & 0.4709 & 0.5109 & 0.7306 \\
\hline & $\leq 3$ & 13 & $\leq 451$ & 1.5 & 1.38 & $\leq 0.118$ & 0.3623 & $\leq 2.352$ & 0.5342 \\
\hline \multicolumn{10}{|l|}{ Catch basin } \\
\hline $0 \mathrm{M}-21$ & $\leq 2$ & 18 & 263 & & 11.33 & $\leq 0.103$ & 0.2929 & 0.308 & 0.3531 \\
\hline CB- & $\leq 1$ & 14 & 334 & 4.1 & 13.37 & 0.1791 & & & 0.5896 \\
\hline $2-17$ & 11 & 18 & 333 & 4.66 & 12.78 & $\leq 0.1758$ & 529 & 0.63 & 0.6565 \\
\hline & 9 & 10 & 75 & 1.4 & 10.68 & & & 0.34 & 0.8454 \\
\hline$C B$ & $\leq 2$ & 16 & 133 & 17.1 & 11.44 & $\leq 0$. & & 0.8766 & 0.6371 \\
\hline & 8 & 11 & & 44.6 & 12.7 & & & 1.1 & $\leq 1.474$ \\
\hline CB & 1.5 & 10 & 19.5 & 0.7 & 8.66 & $\leq 0.1676$ & 0.4 & 0.34 & $\leq 0.8956$ \\
\hline $01 / 2$ & 2.5 & 11 & 25.5 & 12.9 & 6.2 & & & 0.77 & 0.64 \\
\hline $\mathrm{CB}$ & 0 & 14 & 173 & 12.8 & 14.92 & $\leq 0.2$ & & 0.71 & $\leq 0.9498$ \\
\hline & 6 & 11 & 196 & & 278 & & & 0.38 & $\leq 0.643$ \\
\hline CB-2 & 1.5 & 11 & 17.5 & 0.4 & 7.404 & $\leq 0$. & & $\leq 0.48$ & $\leq 1.27$ \\
\hline & 9 & 17 & 314 & 40 & 6.27 & & & 1.2 & 3.681 \\
\hline CB-2 & 6 & 10 & 2548 & 1.9 & 9.12 & $\leq 0.1$ & & 0.485 & 0.7721 \\
\hline & & 20 & 358 & & 11.65 & $\leq 0.0$ & & 0.41 & 0.6464 \\
\hline CB-29 & 1 & 20 & 2719 & & 8.61 & $\leq 0.1092$ & & 0.51 & 0.7712 \\
\hline & 1 & 5 & 12.5 & & & & & 0.47 & 2294 \\
\hline CB-310M-30 & 1.5 & 11 & 478 & 14.8 & 7.2 & $\leq 0.2$ & & 0.51 & $\leq 0.7798$ \\
\hline & 1 & & 1467 & 14 & 7.965 & & & 0.6 & 3444 \\
\hline CB-320-M2 & 1.5 & 16 & 799 & 11.2 & 9.7 & $\leq 0.15$ & & 0.45 & 0.7326 \\
\hline & 2 & 14 & 1408 & 54.2 & 7.5 & & & & 1.033 \\
\hline CB-340C-35 & & 15 & 115 & 1.2 & & & & & 0.612 \\
\hline & 1 & 8 & & 1 & & & & 0.62 & $\leq 0.7556$ \\
\hline CB-360G-20 1/2 & 5 & 19 & 292 & 9.1 & 3.879 & $\leq 0$. & & 0.94 & 1.856 \\
\hline & 1 & 6 & 12 & 1.4 & 7.462 & & & 0.5067 & 0.527 \\
\hline CB-390R-14 1/2 & 1.5 & 13 & 373 & 17 & 8.318 & $\leq 0.14$ & & 0.40 & $\leq 0.7122$ \\
\hline CB-410D-014 & 1 & 9 & 349 & 37.9 & 19.08 & $\leq 0.6238$ & $\leq 1.422$ & $\leq 1.18$ & $\leq 3.302$ \\
\hline$-410 E-044$ & 5 & 17 & 880 & 1.4 & 8.376 & 0.2907 & $\leq 0.2902$ & & $\leq 0.6262$ \\
\hline$-420 D-024$ & 2.5 & 7 & 440 & 18.7 & 5.09 & $\leq 0.4194$ & $\leq 0.812$ & 0.7301 & $\leq 2.072$ \\
\hline CB-420F-27 & 2 & 9 & 671 & 18.1 & 6.308 & 0.3697 & $\leq 0.3634$ & 0.578 & $\leq 0.8412$ \\
\hline$-430 T-86.5$ & 8 & 15 & 1378 & 4.3 & 6.044 & $\leq 0.1986$ & 0.291 & 0.5742 & $\leq 0.8478$ \\
\hline$-460-C$ & 1 & 18 & 86 & 2.3 & 11.62 & $\leq 0.1744$ & 0.4526 & $\leq 0.3564$ & $\leq 0.9554$ \\
\hline CB-470-P & 6 & 17 & 235 & 3.5 & 11.74 & $\leq 0.1146$ & 0.3833 & 0.8328 & 0.9668 \\
\hline CB-470-P2 & 1.5 & 17 & 165 & 31 & 10.51 & $\leq 0.09006$ & & 0.76 & \\
\hline$C B-490-X-36$ & & 11 & 117 & 17 & 6.354 & 0.09741 & 0.2 & 0.4165 & 0.4888 \\
\hline CB-510B-30 & 1 & 14 & 2403 & 16.39 & 34.5 & $\leq 0.7756$ & $\leq 1.526$ & $\leq 1.28$ & $\leq 2.726$ \\
\hline CB-510D-20 & 6 & 10 & 1542 & & $\leq 1056$ & $\leq 66.61$ & $\leq 120.2$ & $\leq 84.87$ & \\
\hline & & & & & 0.607 & & & $\leq 0.1406$ & \\
\hline CB-560G-14 & & & 361 & & 9.552 & $\leq 0.1414$ & $\leq 0.3362$ & 0.7006 & $\leq 0.9168$ \\
\hline CB-590-Q1-12 & 7 & 19 & 2026 & 12.8 & 13.4 & 0.4185 & 0.372 & 0.7338 & 0.9922 \\
\hline CB-610A-54 & 7 & 12 & 18 & 12.1 & 8.682 & 0.405 & $\leq 0.7869$ & 0.8647 & 0.829 \\
\hline CB-610B-16 & 1 & 8 & 1067 & & 4.401 & $\leq 0.05254$ & 0.1961 & 0.3499 & $\leq 0.374$ \\
\hline CB-620J-01-34 & 0.5 & 12 & 892 & & 6.366 & $\leq 0.0702$ & 0.325 & 0.4738 & 0.4028 \\
\hline CB-620J-34 & & 10 & 1329 & & 5.536 & $\leq 0.05042$ & $\begin{array}{r}0.2995 \\
0.1310\end{array}$ & 0.4691 & 0.5174 \\
\hline & & & & & & & $\leq 0.1319$ & & \\
\hline
\end{tabular}


Table D-4. Radionuclide analysis for 1991 sediment study in $\mathrm{pCi} / \mathrm{g}$, unless otherwise noted (continued).

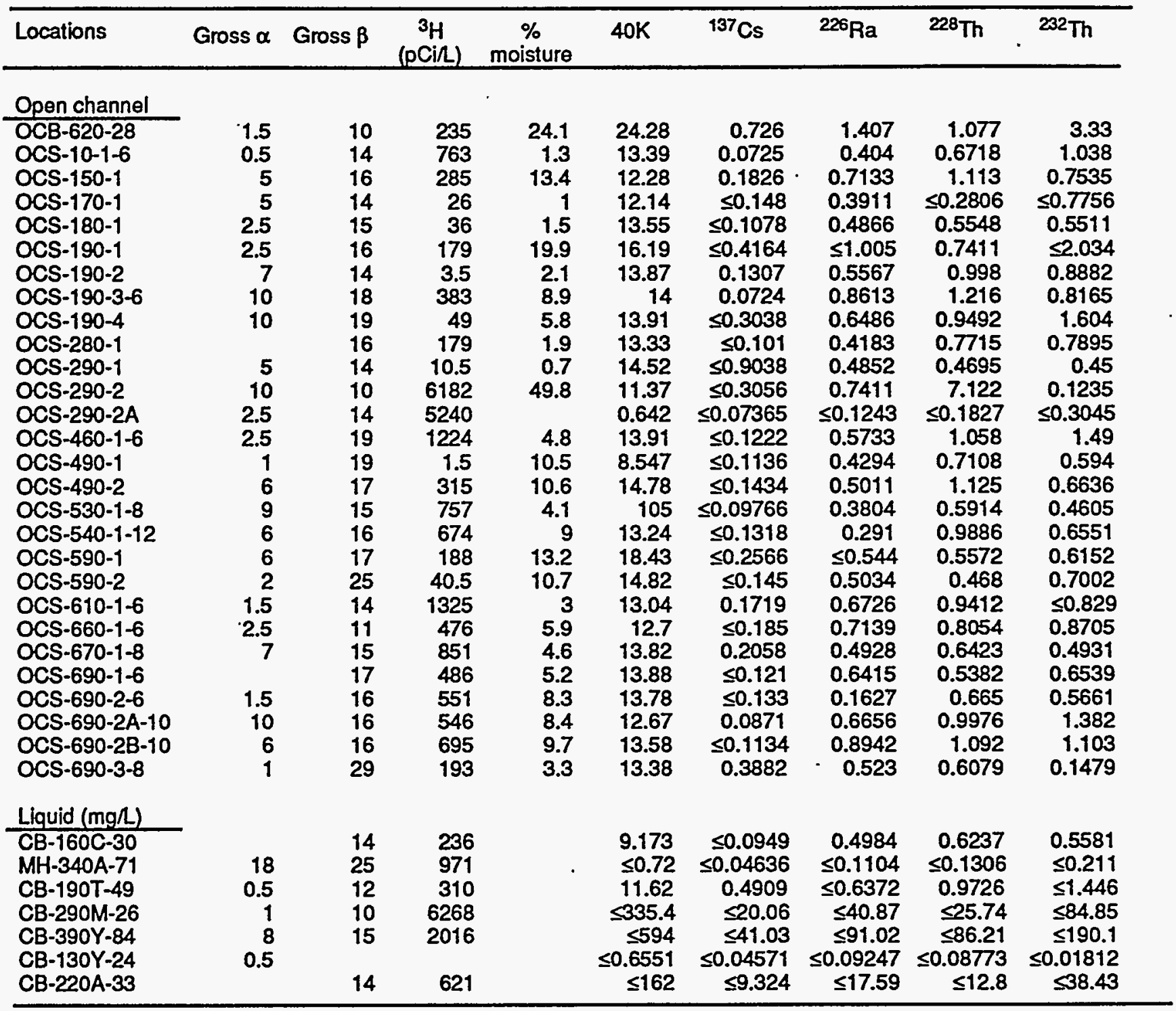


Table D-5. Organic compounds detected by EPA Method 8270 in 1991 study, $\mathrm{mg} / \mathrm{kg}$ unless otherwise noted.

\begin{tabular}{|c|c|c|c|c|c|c|c|c|c|c|c|}
\hline Locations & $\begin{array}{l}\text { 1,3-Di- } \\
\text { chloro- } \\
\text { ben- } \\
\text { zene }\end{array}$ & $\begin{array}{c}\text { 1,4-Di- } \\
\text { chiloro- } \\
\text { ben- } \\
\text { zene }\end{array}$ & $\begin{array}{c}2- \\
\text { Chlor- } \\
\text { ophe- } \\
\text { nol }\end{array}$ & $\begin{array}{c}\text { 4- } \\
\text { Methyl } \\
\text { phenol } \\
\text { (p-Cre- } \\
\text { sol) }\end{array}$ & $\begin{array}{l}\text { 4- } \\
\text { Nitro- } \\
\text { phenol }\end{array}$ & $\begin{array}{l}\text { Acen- } \\
\text { aph- } \\
\text { thene }\end{array}$ & $\begin{array}{l}\text { Anthra } \\
\text { cone }\end{array}$ & $\begin{array}{c}\text { Benzo } \\
\text { (a)an- } \\
\text { thra- } \\
\text { cene }\end{array}$ & $\begin{array}{l}\text { Benzo } \\
\text { (a)py- } \\
\text { rene }\end{array}$ & $\begin{array}{l}\text { Benzo } \\
\text { (b)fluor } \\
\text { an- } \\
\text { thene }\end{array}$ & $\begin{array}{c}\text { Benzo } \\
\text { (g,h,i)- } \\
\text { pery- } \\
\text { lene }\end{array}$ \\
\hline \multicolumn{12}{|l|}{ Background } \\
\hline $\begin{array}{l}\text { BS-5-6 } \\
\text { BS-6-6 } \\
\text { BS7-6 }\end{array}$ & & & & & & & & 0.5 & 0.7 & 0.8 & \\
\hline \multicolumn{12}{|l|}{ Catch basin } \\
\hline \multicolumn{12}{|l|}{ CB-120M-21 } \\
\hline $\begin{array}{l}\text { CB-120S-01-17 } \\
\text { CB-120S-02-17 }\end{array}$ & & & & & & & & 0.3 & 0.2 & 0.5 & \\
\hline $\begin{array}{l}\text { CB-290F-38 } \\
\text { CB-290J-82 } \\
\text { CB-320F-17 }\end{array}$ & & & & & & & & 2 & $\begin{array}{l}3 \\
0.5 \\
50\end{array}$ & $\begin{array}{l}5 \\
0.4\end{array}$ & \\
\hline CB-360G-20 $1 / 2$ & & & & & & & & 4 & 6 & 10 & \\
\hline $\begin{array}{l}\text { CB-390R-14 1/2 } \\
M H-110 G-93\end{array}$ & & & & & & 0.6 & 0.7 & $\begin{array}{l}1.9 \\
0.9\end{array}$ & $\begin{array}{l}5.4 \\
0.7\end{array}$ & $\begin{array}{l}4.4 \\
1.5\end{array}$ & $\begin{array}{l}1.9 \\
0.6\end{array}$ \\
\hline \multicolumn{12}{|l|}{ Open channel } \\
\hline OCS-170-1 & & & & & & & & 0.26 & 0.2 & 0.46 & 0.24 \\
\hline OCS-190-2 & & & & & & & & & & 0.08 & \\
\hline $\begin{array}{l}\text { OCS }-190-3-6 \\
\text { OCS }-280-1\end{array}$ & 0.03 & 0.03 & 0.03 & & & & & & & & \\
\hline OCS-660-1-6 & & & & & 1 & & & & & & \\
\hline \multicolumn{12}{|l|}{ Liquid ( $\mu \mathrm{g} / \mathrm{L}$ ) } \\
\hline $\begin{array}{l}\text { CB-510D-20 } \\
\text { CB-943 }\end{array}$ & & & & 70 & & & & & & & \\
\hline $\begin{array}{l}\text { MH-340A-71 } \\
\text { CB-390Y-84 }\end{array}$ & & & & & & & 2 & 17 & 21 & 20 & 16 \\
\hline
\end{tabular}


Table D-5. Organic compounds detected by EPA Method 8270 in 1991 study, mg/kg unless otherwise noted. (continued)

\begin{tabular}{|c|c|c|c|c|c|c|c|c|c|c|c|}
\hline Locations & $\begin{array}{l}\text { Benzo } \\
\text { (k)fluor } \\
\text { an- } \\
\text { thene }\end{array}$ & $\begin{array}{l}\text { Chry- } \\
\text { sene }\end{array}$ & $\begin{array}{c}\text { Di- } \\
\text { benzo- } \\
(a, h)- \\
\text { anthra } \\
\text { cene } \\
\end{array}$ & $\begin{array}{l}\text { Fluor- } \\
\text { an- } \\
\text { thene }\end{array}$ & $\begin{array}{l}\text { Fluo- } \\
\text { rene }\end{array}$ & $\begin{array}{l}\text { Indeno } \\
(1,2,3- \\
c, d)- \\
\text { pyrene }\end{array}$ & $\begin{array}{c}\mathrm{N}- \\
\text { Nitroso } \\
\text { dime- } \\
\text { thyl- } \\
\text { amine } \\
\end{array}$ & $\begin{array}{l}\text { Phen- } \\
\text { an- } \\
\text { threne }\end{array}$ & Phenol & $\begin{array}{l}\text { Py- } \\
\text { rene }\end{array}$ & $\begin{array}{l}\text { Bis(2- } \\
\text { chloro } \\
\text { ethyl- } \\
\text { jether }\end{array}$ \\
\hline \multicolumn{12}{|l|}{ Background } \\
\hline $\begin{array}{l}\text { BS-5-6 } \\
\text { BS-6-6 } \\
\text { BS7-6 }\end{array}$ & 0.8 & 0.6 & & $\begin{array}{l}1.1 \\
0.6\end{array}$ & & & & $\begin{array}{l}1.2 \\
0.6\end{array}$ & & $\begin{array}{l}1.1 \\
0.6\end{array}$ & \\
\hline \multicolumn{12}{|l|}{ Catch basin } \\
\hline $\begin{array}{l}\text { CB-120M-21 } \\
\text { CB-120S-01-17 } \\
\text { CB-120S-02-17 }\end{array}$ & 0.5 & 0.3 & & $\begin{array}{l}0.3 \\
0.5\end{array}$ & & & & 0.3 & & $\begin{array}{l}0.4 \\
0.5\end{array}$ & \\
\hline $\begin{array}{l}\text { CB-29OF-38 } \\
\text { CB-290J-82 } \\
\text { CB-320F-17 }\end{array}$ & $\begin{array}{l}5 \\
0.4\end{array}$ & $\begin{array}{l}4 \\
0.3\end{array}$ & & $\begin{array}{l}6 \\
0.4 \\
2\end{array}$ & & & & $\begin{array}{l}4 \\
0.4\end{array}$ & & $\begin{array}{l}7 \\
0.4 \\
2\end{array}$ & \\
\hline $\begin{array}{l}\text { CB-360G-20 } 1 / 2 \\
\text { CB-390R-14 } 1 / 2 \\
\text { MH-110G-93 }\end{array}$ & $\begin{array}{l}10 \\
4.4 \\
1.5\end{array}$ & $\begin{array}{l}4 \\
4.6 \\
0.8\end{array}$ & & $\begin{array}{l}8 \\
4.8 \\
1.3\end{array}$ & 0.4 & $\begin{array}{l}1.6 \\
0.5\end{array}$ & & $\begin{array}{l}4 \\
5 \\
0.7\end{array}$ & & $\begin{array}{l}7 \\
5.4 \\
1.2\end{array}$ & \\
\hline \multicolumn{12}{|l|}{ Open channel } \\
\hline \multirow{2}{*}{$\begin{array}{l}\text { OCS-170-1 } \\
\text { OCS-190-2 } \\
\text { OCS-190-3-6 } \\
\text { OCS-280-1 } \\
\text { OCS } 660-1-6\end{array}$} & $\begin{array}{l}0.46 \\
0.08\end{array}$ & $\begin{array}{l}0.3 \\
0.09\end{array}$ & 0.22 & $\begin{array}{l}0.54 \\
0.09\end{array}$ & & 0.2 & \multirow{2}{*}{0.03} & $\begin{array}{l}0.29 \\
0.08\end{array}$ & \multirow{2}{*}{0.03} & 0.49 & \multirow{2}{*}{0.03} \\
\hline & & 0.2 & & 0.2 & & & & & & 0.2 & \\
\hline $\begin{array}{l}\text { Liquid }(\mu \mathrm{g} / \mathrm{L}) \\
\text { CB-510D-20 } \\
\text { CB-943 }\end{array}$ & & & & & & & & & & & \\
\hline $\begin{array}{l}\text { MH-340A-71 } \\
\text { CB-390Y-84 }\end{array}$ & 20 & 17 & 12 & $\begin{array}{l}27 \\
20\end{array}$ & & & & $\begin{array}{l}21 \\
20\end{array}$ & & $\begin{array}{l}24 \\
20\end{array}$ & \\
\hline
\end{tabular}


Table D-6. Pesticides detected in 1991 study (EPA Method 8080), $\mathrm{mg} / \mathrm{kg}$ unless otherwise noted.

\begin{tabular}{|c|c|c|c|c|c|c|c|c|c|}
\hline Locations & Dieldrin & p,p'-DDE p,p'-DDT & $\begin{array}{l}\text { Endo- } \\
\text { sulfan I }\end{array}$ & $\begin{array}{l}\text { Endo- } \\
\text { sulfan II }\end{array}$ & $\begin{array}{l}\text { Endo- } \\
\text { sulfan } \\
\text { sulfate }\end{array}$ & $\begin{array}{l}\text { Hepta- } \\
\text { chlor } \\
\text { epoxide }\end{array}$ & $\begin{array}{l}\text { Meth- } \\
\text { oxy- } \\
\text { chlor }\end{array}$ & $\begin{array}{c}\text { BHC, } \\
\text { beta } \\
\text { isomer }\end{array}$ & $\begin{array}{c}\text { BHC, } \\
\text { delta } \\
\text { isomer }\end{array}$ \\
\hline
\end{tabular}

Background

BS-6-6

BS7-6

Catch basin

CB-140J-13

CB-1900-10 1/2

CB-350E-44

CB-360G-20 1/2

CB-410E-044

CB-420D-024

CB-590-Q1-12

MH-110G-93

Open channel

OCS-150-1

OCS $-460-1-6$

OCS-660-1-6
0.14

0.006

Liquid $(\mu g / L)$

CB-390Y-84

0.098

0.01

0.19

0.02
0.04

0.1

0.006

0.02

0.12

0.3
0.43

0.17 
Table D-7. Volatile organic compounds detected in 1991 study, values in $\mathrm{mg} / \mathrm{kg}$ unless otherwise noted.

\begin{tabular}{|c|c|c|c|c|c|c|c|c|c|c|c|c|c|}
\hline Locations & $\begin{array}{l}\text { 1,2-Di- } \\
\text { chloro- } \\
\text { ethene }\end{array}$ & $\begin{array}{l}\text { Ace- } \\
\text { tone }\end{array}$ & $\begin{array}{l}\text { Chloro- } \\
\text { form }\end{array}$ & $\begin{array}{l}\text { Ethyl } \\
\text { ben- } \\
\text { zene }\end{array}$ & $\begin{array}{c}\text { Froon } \\
113\end{array}$ & TCA & TCE & $\begin{array}{c}\text { Tri- } \\
\text { chloro- } \\
\text { fluoro- } \\
\text { me- } \\
\text { thane }\end{array}$ & $\begin{array}{c}\text { Tolu- } \\
\text { ene }\end{array}$ & PCE & $\begin{array}{c}\text { Total } \\
\text { xylene } \\
\text { iso- } \\
\text { mers }\end{array}$ & $\begin{array}{l}\text { cis-1,2- } \\
\text { di- } \\
\text { chloro } \\
\text { ethene }\end{array}$ & $\begin{array}{c}\text { Vinyl } \\
\text { chlor- } \\
\text { ide }\end{array}$ \\
\hline \multicolumn{14}{|l|}{ Background } \\
\hline BS-1-02-6 & & & & & & & & & 0.005 & & & & \\
\hline BS-2-8 & & & & 0.01 & & & & & 0.03 & & 0.039 & & \\
\hline BS-3-6 & & & & & & & & & 0.006 & & & & \\
\hline BS-4-6 & & & & & & & & & 0.006 & & & & \\
\hline BS-6-6 & & & & & & & & & 0.009 & & 0.008 & & \\
\hline \multicolumn{14}{|l|}{ Catch basin } \\
\hline CB-120S-02-17 & & & & & & & & & & 0.017 & & & \\
\hline CB-170B-18 & & & & & & & & & 0.012 & & & & \\
\hline CB-280D-24 & & & & & & & & & & 0.007 & & & \\
\hline CB-29OF-38 & & & & 0.016 & & & & & 0.027 & & 0.064 & & \\
\hline CB-290J-82 & & & & & & & & & 0.045 & & & & \\
\hline CB-310C-24 & & & & & & & & & & & & & 0.011 \\
\hline CB-320F-17 & 0.03 & 0.067 & & & & & & & & 0.007 & 0.005 & 0.03 & 280 \\
\hline CB-350E-44 & & & & & & & & & 0.03 & & 0.012 & & \\
\hline CB-360J-48 & & & & & & & & & 0.009 & & 0.007 & & \\
\hline CB-410E-044 & & & & 0.03 & & & & & 0.1 & & 0.089 & & \\
\hline CB-430T-86.5 & & & & 0.013 & 0.015 & & 0.005 & 0.005 & 0.35 & 0.011 & 0.01 & & \\
\hline CB-620J-01-34 & & & & & & & & & & 0.27 & & & \\
\hline CB-620J-34 & & & & & & & & & & 0.22 & & & \\
\hline \multicolumn{14}{|l|}{ Open channel } \\
\hline OCS-190-2 & & & & 0.008 & & & & & 0.038 & & 0.026 & & \\
\hline OCS-290-2 & & 0.1 & & & & & & & & & & & \\
\hline OCS-460-1-6 & & & & & & & & & & & 0.01 & & \\
\hline OCS-590-2 & & & 0.023 & & & & & & & & & & \\
\hline OCS-660-1-6 & & & & 0.006 & & & & & & & 0.024 & & \\
\hline OCS-670-1-8 & & & & & & & & & 0.016 & & 0.008 & & \\
\hline OCS-690-2B-10 & & & & & & & & & & & 0.016 & & \\
\hline OCS-690-3-8 & & & & & & & & & & & 0.021 & & \\
\hline \multicolumn{14}{|l|}{ Liquid ( $\mu g / L)$} \\
\hline CB-510D-20 & & 0.11 & & & & & & & 0.036 & & & & \\
\hline CB-520F-76 & & & 1.3 & & 0.6 & & 0.7 & & & 4.9 & & 0.9 & \\
\hline CB-943 & & & 1.5 & & & 32 & & 1.5 & & & & & \\
\hline MH-340A-71 & & & 5.7 & & & & & & & & & & \\
\hline CB-290M-26 & & 26 & & & & & & & & & 2.1 & & \\
\hline CB-390Y-84 & & 13 & & &. & & & & & & & & \\
\hline CB-220A-33 & & & & & & & & & 67 & & & & \\
\hline
\end{tabular}

D-9 